Hershkovitz

Montiaceae Ecological Evolution

\title{
Perspectives from Montiaceae (Portulacineae) evolution. II. Ecological evolution, phylogenetic comparative analysis, and the Principle of Evolutionary Idiosyncraticity
}

\author{
Mark A. Hershkovitz ${ }^{1}$ \\ ${ }^{1}$ Santiago, Chile \\ cistanthe@gmail.com
}

\section{ABSTRACT}

The present paper reviews evidence for ecological evolution of Montiaceae. Montiaceae (Portulacineae) comprise a family of ca. 275 species and ca. 25 subspecific taxa of flowering plants distributed mainly in extreme western America, with additional endemism elsewhere, including other continents and islands. They have diversified repeatedly across steep ecological gradients. Based on narrative analysis, I argue that phylogenetic transitions from annual to perennial life history have been more frequent than suggested by computational phylogenetic reconstructions. I suggest that a reported phylogenetic correlation between the evolution of life history and temperature niche is coincidental and not causal. I demonstrate how statistical phylogenetic comparative analysis (PhCA) missed evidence for marked moisture niche diversification among Montiaceae. I discount PhCA evidence for the relation between Montiaceae genome duplication and ecological diversification. Based on the present analysis of Montiaceae evolution, I criticize the premise of the prevalent statistical approach to PhCA, which tests Darwinian deterministic hypotheses against stochastic evolutionary null models. I discuss theoretical/empirical evidence that evolution is neither stochastic, nor Darwinistically-determined, but idiosyncratic. Idiosyncraticity describes the outcome of a stochastically perturbed nonlinear chaos-like process. The Principle of Evolutionary Idiosyncraticity (PEI) is based on the evolutionary theory of Natural Drift, which maintains that determinism in evolution is a property of the organism and not, as maintained by the theory of Natural Selection, its traits or its milieu. This determinism is characteristic of chaotic functions, which are absolutely determinate, generate self-similarity, but remain absolutely unpredictable. PEI explains precisely observations that evolution proceeds not linearly, but chaotically, producing both quasi-linear fractal-like patterns and non-linear jumps. PEI has ramifications for all areas of macroevolutionary research. In particular, it demonstrates both the fallacy and futility of the statistical PhCA approach that interprets evolutionary causes in terms of evolutionary correlations. However, statistical methods of PhCA can be applied heuristically and fruitfully to reveal idiosyncraticity and discover 
Hershkovitz

Montiaceae Ecological Evolution

evolutionary novelty. This, in turn, is demonstrated by the emergence of statistical anomalies in evolutionary analyses of Montiaceae.

KEY WORDS: Montiaceae, life history, climate niche, polyploidy, phylogenetic comparative analysis (PhCA), natural selection (NS), natural drift (ND), chaos, stochasticity, determinism, principle of evolutionary idiosyncraticity (PEI).

\section{BACKGROUND}

Montiaceae (Portulacineae) are an angiosperm clade comprising about 275 species plus about another 25 accepted subspecific taxa (updated from [1]). Montiaceae are sister to remaining Portulacineae, which include seven other families and 1600-2000 species, ca. 90\% of which belong to Cactaceae [2]. Montiaceae split from other Portulacineae ca. 35-40 million years ago [3, 4]. The first part of this two-part work on Montiaceae evolution [5] critically reviewed phylogenetic and phylogeographic evidence. That work considered inevitable the nonconformance of at least some phylogenetic/phylogeographic data to whatever model/criterion might be applied to reconstruct them. This inevitable idiosyncratic data behavior was attributed not to error, omission, ambiguity, variance, or noise, but to the idiosyncraticity of evolution itself.

Thus, the Principle of Evolutionary Idiosyncraticity (PEI) was introduced to argue that linear monotonic model/criterion-based methods cannot recover or predict evolutionary history accurately or, in any case, better than hypothetico-deductive narrative analysis. At the same time, part one of this work concluded that quantitative analytical methods have tremendous heuristic value if they are used, specifically, to explore and discover idiosyncraticity. Several examples were elaborated, e.g., using cladistic conflict and branch-length asymmetries to identify a likely ancient polyploid hybrid that, in turn, behaves idiosyncratically relative to widely accepted dogma on the evolutionary consequences of polyploidization and hybridization.

The present paper extends this argument to the analysis of ecological and phenotypic macroevolution and diversification, in particular the testing of macroevolutionary hypotheses. Montiaceae phenotypic and ecological diversification evolution has attracted interest because of peculiarities rendered evident by phylogenetic resolution. Montiaceae species distinguish from other Portulacineae phylogeographically, ecologically, and phenotypically. Their distribution concentrates in the high-relief and climatically diverse temperate zone westwards from the American high cordillera (cis-cordilleran), with lesser additional endemic diversity east of the American cordillera (trans-cordilleran), Siberia, Australia, New Zealand, and the Kerguelen Islands [1, 5]. Correspondingly, Montiaceae species concentrate especially in cooler/moister (higher altitude and/or latitude) and warmer/drier Mediterranean and desert environments [3, 5]. They also differ from other Portulacineae in comprising mostly hemicryptophytic (including acaulescent rosettiform-caudiciform) perennials and therophytes $[1,3,6]$. 
Hershkovitz

Montiaceae Ecological Evolution

Other Portulacineae are distributed mainly more towards the tropics and from the American high cordillera eastwards (trans-cordilleran) and Africa/Madagascar, with a few taxa in warmer parts of Australasia/Oceania $[3,5,6]$. They are largely phanerophytic herbs, shrubs, trees, and vines, and often stem succulents [2, 3]. However, (most?) Portulaca (Portulacaceae) species are monocarpic annuals [7] and most Anacampserotaceae species are hemicryptophytes, a few acaulescent $[8,9]$.

Using various methods of statistical phylogenetic comparative analysis (PhCA), Ogburn \& Edwards [3] (heretofore "OE") studied ecological evolution of Montiaceae, especially the relation between life history and temperature and moisture niche. They reported an increased rate of phylogenetic diversification of temperature niche and life history relative to other Portulacineae. Montiaceae have incurred ca. 14 life history transitions since the split between Cistantheae and Montioideae, 25-35 million years ago, out of the total ca 16 transitions among all Portulacineae over 50+ million years [3]. OE [3] argued that the two trends were related causally.

$\mathrm{OE}$ [3] attributed phylogenetic life history lability of Montiaceae to their ancestral herbaceous and, in particular, hemicryptophytic growth form. Phylogenetic niche breadth lability itself had been ascribed to herbaceous more than woody growth forms [10]. But [3] argued that the rosettiform hemicryptophytic form of Montiaceae permitted phylogenetic lability in relative above- versus below-ground biomass allocation. This, in turn, permitted Montiaceae to adapt phylogenetically to a broader range of climate conditions. Meanwhile, McIntyre [11] and Smith et al. [12] performed statistical analyses to evaluate the prediction that ecological diversification among Montiaceae is (alternatively) consequent to whole genome duplication (polyploidy).

Below, the statistical results are evaluated in terms of theoretical and methodological assumptions and in light of taxon-by-taxon narrative analysis. I demonstrate that statistical PhCA has tremendous heuristic and systematic descriptive value. But, like statistical phylogenetic reconstruction methods, the methodological assumptions and parameterizations tend to impose as much as discover evolutionary order [5]. I demonstrate how narrative analysis optimizes the heuristic value of $\mathrm{PhCA}$. In particular, $\mathrm{PhCA}$ provides a platform for narrative analysis that facilitates discovery of evolutionary idiosyncrasy. Taxonomy of Montiaceae in this work follows [1, $5]$.

PHYLOGENETIC COMPARATIVE ANALYSES OF ECOLOGICAL EVOLUTION OF MONTIACEAE Introduction to phylogenetic comparative analysis: the statistical Darwinian paradigm at the macroevolutionary scale

Within the past three decades, PhCA has established as the principal paradigm of macroevolutionary analysis [13, 14]. The paradigm traces to a seminal paper by Felsenstein [15]. Up to that point, statistical analyses of evolutionary adaptation commonly interpreted taxa, especially species, as ideal observations (independent and 
Hershkovitz

Montiaceae Ecological Evolution

identically distributed). Felsenstein emphasized that they are not, because they are (phylo-)genetically related. Thus, similarity of organisms might owe to independent parallel evolution (the classical assumption) or to phylogenetic autocorrelation. In the latter case, similarity among taxa is not independent.

Thus, the PhCA paradigm purported to "correct for phylogeny" in statistical analysis of macroevolutionary variables, because phylogeny predicts that variable values should be autocorrelated phylogenetically. For example, a phylogenetically naïve statistical analysis might show that certain taxa are "bigger" in a particular environment, and conclude that the environment is the cause. But another explanation is that those taxa inherited bigness from a big common ancestor, and that the environment played no role in the evolution of size. Thus, the PhCA paradigm purported to develop phylogeny-aware objective quantitative functions for testing narrative evolutionary hypotheses. Alternatively, the environment is implicated if bigness evolved in several taxa phylogenetically independently.

The PhCA paradigm generally purports to extend the Darwinian adaptationist statistical population genetic paradigm to the phylogenetic level. In particular, it purports to analyze evolutionary determinism causally in relation to one or another biotic or abiotic parameter. This is the generic premise of the research discussed here. Such biotic/abiotic parameters then are conceived as possible Darwinian drivers or determinants of evolution. This premise will be challenged in the final discussion.

As simple and elegant as could be Felsenstein's [15] observation, its solution could not be more complex. Subsequent theoretical and methodological development up to the present day has raised certain theoretical and practical questions. One is the relevance of evolutionary history of one lineage in explaining/predicting the fate of another [16]. More generally, this question ultimately maps to the validity of the Darwinian adaptationist statistical population genetic paradigm. The theme will reemerge later in this work. Since the statistical paradigm predominates in evolutionary biology, discussion below will start with the supposition that it is valid.

But even within the statistical paradigm, a difficult question remains and can be encapsulated as "how independent is phylogenetically independent?" The problem traces to the mathematical peculiarity of phylogeny. The connectedness of phylogeny renders it a unitary entity [17]. Moreover, it is a tree not only metaphorically, but also literally, albeit disarticulated [18]. In particular, all organisms are like organs developed from a single cell along divergent germlines. This renders impossible true independence among terminal branches, not coincidentally called "leaves." Thus, an analogous question is: at what point and to what degree can literal plant leaves develop independently of the genetic and developmental history of the plant that bears them? Evidence from plant development suggests that the answer is never. The practical implication for PhCA is that analyzed individual traits might seem to arise phylogenetically independently, but nonetheless might be phylogenetically canalized.

In the period following [15] and up to the present, theory and methods have been developed to carry out statistical macroevolutionary analysis that corrects for phylogenetic autocorrelation. The estimated degree of 
Hershkovitz

Montiaceae Ecological Evolution

independence from phylogeny varies with PhCA method, although all estimation methods ultimately appeal to a presumed stochasticity of evolution. The most primitive methods of "phylogenetic independent contrasts" were nonparametric and assumed tacitly that only cladistic branching order affect the stochastic likelihood of phenotypic trait distributions [19]. Parametric methods estimate phylogenetic independence as a function of phylogenetic divergence amount/time estimated from molecular marker tree branch lengths [19]. Some currently popular methods solve the independence question by modeling trait evolution as a Brownian motion random walk, where the probability of trait state change is estimated from the observed frequency in a time-calibrated molecular phylogenetic tree. This method determines whether phylogenetic trait distributions could have evolved by chance. This, in turn, involves assumptions and/or additional calculations of a relation between molecular marker and phenotypic evolution, which itself may change over time [20,21]. Each mathematical intervention introduces method- and data-specific assumptions and/or artifacts. As in molecular phylogenetic reconstruction, each necessary model parameter addition is counterproductive statistically, creating a countertendency to prefer deceptively precise but false models [16].

Existing PhCA methods assume strictly cladogenetic phylogenesis and behave best with fully and unequivocally resolved phylogenies. Increasing evidence for high, if not ubiquitous, incidence of reticulation and/or genetic lineage sorting throughout the course of plant evolutionary history (references to numerous too cite) possibly undermines the entire PhCA paradigm. While Bayesian estimation methods render computationally tractable the inclusion of reticulation and lineage-sorting parameters, the consequent degeneration of statistical phylogenetic certainty likely would suffer severely. Tractability of phylogenetic trait evolution might be impossible. Indeed, cladogenetic and branch length ambiguity alone can mislead some PhCA methods [22].

Not broadly appreciated is that, while PhCA purports to study evolution, the approach itself is requisite to the statistical and not necessarily the evolutionary biological research program. A different school of hypotheticodeductive evolutionary biology questions the relevance of statistical correlations in evolutionary interpretation, since it implies that evolutionary causation in one organism is determined not biologically, but statistically [16]. Wenzel \& Carpenter [16] effectively argued that frequencies of evolutionary events are incidental consequences of evolutionary histories and not indicative of evolutionary constraints or causes. They also argued that random null models applied in PhCA are illegitimate.

More generally, the arguments of [16] root in criticism of the statistical research program in science generally [23]. This criticism is existential. It rejects the very premise of statistical PhCA, regardless of methodspecific assumptions and analysis-specific errors, omissions, ambiguities, variance, or noise. This does not mean that PhCA conclusions are false. They might be true, ironically, by chance. Nonetheless, statistical approaches to phylogenetics, phylogeography, and PhCA remain the predominant paradigm in macroevolutionary analysis.

The present work takes a trajectory distinct from the conventional debate. The first part of this work [5] indeed demonstrated the inadequacy of monotonic methodological models/criteria in 
Hershkovitz

phylogenetic/phylogeographic reconstruction, hence also prediction. But this inadequacy applies to all quantitative methods of evolutionary analysis, frequentist or not. Invariably, some data do not behave as the model/criterion would predict. This data misbehavior, termed idiosyncraticity, seemed to be the only feature consistently emergent in all evolutionary analyses. At the same time, it is undeniable that widely accepted and seemingly robust phylogenetic interpretations have been derived methodologically using both frequentist and non-frequentist models/criteria. Otherwise, the phylogenetic arguments presented in this and all of my previous work would be baseless. The arguments might be false, but they are not baseless.

The similarity of phylogenetic results yielded by frequentist and non-frequentist methods deserves comment. This might suggest that idiosyncratic data behavior indeed reflects trivial error, omission, ambiguity, variance, and/or noise. But idiosyncraticity cannot be ignored. As I describe it in this work, idiosyncraticity does not reflect error, omission, ambiguity, variance, and/or noise, although evolutionary analyses invariably include these also. PEI reflects a model of evolution that describes coincident chaos-like and stochastic processes that result in non-Darwinian evolution by "natural drift" (ND; [24]). Thus, idiosyncraticity characterizes not only pathological data behavior in evolutionary analyses, but all data behavior, whether patterned/orderly/lawful/predicted or unpatterned/disorderly/unlawful/unpredicted. Existing evolutionary analytical methods optimize recovery of the former qualities and marginalize the latter. PEI explains both apparent order and disorder. Per PEI, existing PhCA methods provide, at best, (albeit useful) statistical systematic descriptions of evolutionary history, but not, as they predicate, evolutionary explanations or reliable predictions. PEI elicits alternative approaches to evolutionary explanation and prediction that correct not only for phylogenetic autocorrelation, but also for idiosyncraticity.

In summary, when reviewing PhCA works, one must keep in mind a few simple questions whose answers should be obvious. Besides questioning technical data accuracy, one must question the process being analyzed evolution. Is evolution linear, stochastic, and stationary? Does evolution yield independent and identically distributed taxa and traits? Are the possible outcomes of trait evolution limited to two or any finite number? Does increasing phylogenetic sample size reduce variance and converge on a true value? In other words, does the evolutionary process conform to the introductory statistics course model of coin-flipping?

The answers to the preceding question are all negative and, in fact, not further from the truth. Evolution axiomatically refers to a process by which organisms become increasingly and unpredictably different and whose evolutionary trajectories become increasingly unique and irreducible. Otherwise we would not bother to study evolution, nor be here to study it. Even if assumptions of coin-likeness are acceptable in studies of individuals in populations, they are tautologically anathema to studies of taxa in phylogenies. In particular, taxa are diagnosed by their evolved and irreversible tangible differences, which negate their statistical equivalence. This is true at every evolutionary "node," but especially is evident in major biological radiations that have occurred periodically since life's origin. Ancestors of such radiated clades would have been infinitesimally distinct from their brood, yet 
Hershkovitz

Montiaceae Ecological Evolution

their realized evolutionary projections could not have been estimated statistically as a function of trait flipping. Macroevolution is modeled better as coins that change into different coins mid-flip. Over time, they change into dice, with six possible outcomes, then bingo balls, and eventually, at the PhCA scale, gigabyte hard drives.

Effectively, PhCA bases upon hindsight-definition of the sample spaces, i.e., sample space defined in terms of outcomes observed thus far. But departure from this sample space seems to be the rule, if not the essential point, of evolution. For example, among the "peas in the pod" of the angiosperm ancestral species, one gave rise to a single extant rare species restricted to New Caledonia (Amborella trichopoda), and another gave rise to the rest of extant angiosperm diversity. The latter include forms and ecologies not anticipated by Mesozoic gymnosperms.

From a statistical perspective, increasing phylogenetic phenotypic sampling theoretically should increase rather than decrease variance. This is the same as for genotypic sampling, in which increasing sampling actually can confound phylogenetic reconstruction [5, 19]. Thus, in practice, PhCA is expected to "miscorrect" as much as correct for phylogeny: as increased sampling increases the diversity sampled, means of apples naturally become means of apples and oranges. Similar but spatiotemporally remote and contextually distinct observations are pooled as being the same (cf. [23]). Expectedly, the effect aggravates considerably in the analysis of abstract and complex characters such as life histories, growth forms, and "niches." This will emerge later in this discussion.

Per its statistical Darwinian adaptationist basis, PhCA also attributes causality to phylogenetic correlations. OE [3] examined the relation between diversification of climate niche and life history among Montiaceae and explained evolution of the former as a consequence of evolution of the latter. Meanwhile, [11, 12] attempted to explain essentially the same relationship a consequence of polyploidy. Technical accuracy of these results in the context of a statistical framework will be discussed below, while the logical basis for interpreting evolutionary cause on the basis of phylogenetic correlation will be analyzed in the final discussion.

This text is divided into two principal sections. The first, a continuation of [5], critically reviews PhCAs of Montiaceae phenotypic and ecological diversification as a platform for deriving PEI in the second section. To reemphasize this objective, as in [5], the title is "Perspectives from..." rather than "Perspectives on...". The first section is qualitatively similar to an insightful narrative critique of PhCA of weevils [25], also emphasizing sampling issues and observations that do not conform to the analyzed dataset and, hence, conclusions. These exceptions might owe to errors, omissions, and/or ambiguities that undermine but, by themselves, do not invalidate PhCA conclusions. But I argue that many exceptions reflect evolutionary idiosyncrasy, whose implications are more profound. Thus, the second major section of this text elaborates PEI formally and in a broader theoretical and practical context. 
Hershkovitz

Montiaceae Ecological Evolution

OE [3] and Smith et al. [12] applied parametric methods that scale phenotypic evolution to a molecular phylogenetic chronogram estimate, based on phenotypic states observed in the terminals. The methods do not assume necessarily that phenotypic evolution scales linearly with time. Rather, some methods assume an instantaneous rate of phenotypic transformation that can be estimated using the phylogeny. The probability of change is cumulative over time. This logic is identical to that used to estimate DNA substitution models, in turn used to reconstruct phylogenies. The effect of assumption violations in model-based DNA phylogenetic reconstruction was reviewed briefly in [5]. The effect is much greater in PhCA, because every trait incidental to an organism introduces a parameter, and the trait states tend to vary more continuously. Notions of probability and scaling of phenotypic evolution will be discussed later in this work.

Current PhCA methods (applied in [3, 12]) test phylogenetic autocorrelation/independence using a parametric Markov Chain Monte Carlo randomization procedure that simulates stochastic character evolution along branches of a phylogenetic tree and, depending on the question, also simulating the process of phylogenesis itself. Stochastic probabilities are estimated using the distribution of phenotypes among terminals of the "observed" phylogeny. The methods permit estimation of predicted unconstrained trait distributions. Phylogenetic autocorrelation is then the degree to which a single trait clusters phylogenetically more often than expected by phylogenetic chance, given the assumptions and latent parameters of the model. Phylogenetic independence is the inverse. Phylogenetic trait correlation, implicating causal determinism, is deduced when two or more traits associate phylogenetically more than expected by phylogenetic chance.

However, as in phylogenetic reconstruction, there is no escaping the reality that these stochastic evolutionary models are conditioned by a single realized phylogeny imperfectly estimated. It does not and cannot consider the essentially infinite universe of phylogenies that an ancestor might have realized under slightly different circumstances [26]. This cannot help but create a determination bias that, in turn, will tend to impose phylogenetic correlations rather than truly test them. More to the point, this underscores the singularity of phylogeny and the consequent paradox of statistical analysis of a process that, as observations are added, converges on a single observation. This paradox returns in the discussion of PEI.

$\mathrm{OE}$ [3] and Smith et al. [12] demonstrate excellent examples of questionable theoretical assumptions widely incorporated into PhCA in practice. These are besides epistemological assumptions, elaborated later. For example, PhCAs commonly assume that only the investigated variables determine the observed phylogenetic outcome. This is unrealistic (e.g. [23]). For example, [3] and [12] each analyze the evolution of the same variables (climate niche) among Montiaceae, but in relation to different variables (life history and polyploidy). Each analysis tacitly presumes that other trait is irrelevant. This renders naïve and simplistic even PhCA methods applying the most sophisticated of stochastic evolutionary models and algorithms. Interestingly, but not unexpectedly, different PhCAs indeed can "prove" different causes for the same effect. This is common in statistical cause/effect relations [23]. In theory, this problem can be addressed by multivariate statistical methods. 
Hershkovitz

Montiaceae Ecological Evolution

1 But this is problematic, as well. Among other problems, it requires data for all traits in all taxa and requires that

2 all are sufficiently polymorphic phylogenetically to be modeled accurately. And the number of phenotypic traits is

3 infinite, so it is impossible to estimate the evolutionary consequences of all of them.

Both [3] and [12] at least partially addressed phylogenetic uncertainty. PhCA ideally assumes accuracy of a phylogeny globally. In particular, error in any part of the tree affects PhCA calculations throughout the tree. Moreover, PhCA methods are differentially sensitive to phylogenetic uncertainty [22]. OE [3] based their PhCA on their five- rather than three-marker Montiaceae phylogeny but did apply a procedure intended to recover "most credible clades." As discussed in [5], while incorporating more data, the five-marker phylogeny introduced considerable phylogenetic conflict/uncertainty in terms of branch support. Support for branches of interest deteriorated further in Smith et al.'s [12] Montiaceae phylogeny, although this analysis covered all of Caryophyllales and was not optimized for finer scale phylogeny. Phylogenetic uncertainty may be mitigated by whole genome sequencing approaches (e.g., $[4,27]$ ), though in some cases in favor of reticulate rather than cladistic evolution (e.g., [27]). Reticulate evolution of genes implicates reticulate evolution of phenotypic traits, also.

OE [3] also addressed phylogenetic uncertainty by basing their analysis on a sample of 500 final trees generated in their Bayesian phylogenetic analysis. This would incorporate any conflicting topologies in those generations. While the method is better than no correction at all, like its conventional counterparts, the Bayesian method does not correct for all sources of statistical Type I and II error at all nodes. Some of these were described in [5]. In any case, the Bayesian approach itself is prone to Type 1 error, and these errors likely are locked in by the time of the 500 final tree generations. However, for the present discussion, I will assume that the procedures in $\mathrm{OE}$ [3] adequately address phylogenetic uncertainty. My discussion focuses on other assumptions.

$\mathrm{OE}$ [3] and Smith et al. [12] analyzed phylogenetic correlates of climate niche estimated as the calculated species mean of climate database values near collecting localities recorded in the GBIF database at the time their research was undertaken. Climate niche refers to the climate component of the total ecological niche. Climate niche is better interpreted as a complex of "niche traits," analogous to morphological traits. Environmental traits per se are not inherited genetically, but as niche traits, they are part of the extended organismal phenotype. In this sense they are inherited.

The approach of $[3,12]$ exemplifies one of the intended purposes of creating both climate and taxonomic databases. They are intended to facilitate machine-driven analysis and synthesis of massive global-scale data in fields such as ecology and evolution without requiring additional laborious, costly, and/or redundant data gathering. But the databases create uncertainty associated with data completeness and accuracy. Thus, the excessive ease with which these data can be machine-analyzed mindlessly creates the conditions for prominent publication and propagation of unwarranted or even demonstrably false conclusions. This always has been the 
Hershkovitz

Montiaceae Ecological Evolution

hazard of the development of technology that functions independent of the theoretical/practical knowledge and competence to apply it properly (as elaborated later).

While the GBIF taxonomic database is improving daily and has exceptional utility for taxonomic research its adequacy for applied ecological research remains questionable, more so at the time of data collection in [3, 12]. Taxon identification and range representation remain troublesome. This, in turn, partially reflects the "taxonomic problem," the global deemphasis on basic taxonomic and inventory research in favor of, ironically, emphasis on more "scientific" research yielding higher publication "impact factors." [28-30]. In other words, emphasis on development of PhCA, molecular phylogenetics, and other technological approaches to biodiversity study has been at least somewhat self-defeating.

Setting aside the question of taxonomic identity and range accuracy, [3, 12] introduced their own interpretative artifacts that may have influenced their conclusions. One is the recording of climate niche traits as the mean species value calculated for georeferenced collections. These mean values then were used to make intertaxon phylogenetic comparisons. This procedure probably reflects the emergence of the PhCA paradigm from the statistical evolutionary population genetics paradigm. The latter defines evolution as a "change in a mean value of a trait in a population" (see below). In PhCA, this becomes changes of the mean of means among taxa. Regardless of the technical accuracy of this mean, as elaborated in the final section of this work, this statistical definition of evolution has been thoroughly debunked by theoretical and philosophical biologists and it is rejected here. Evolution here is interpreted as change at the level of individuals, not populations, nor taxa.

This interpretative difference has severe consequences in the present context. In the statistical interpretation, similarity/difference of taxa with overlapping polymorphism values is determined by difference of their means/variance. In the PEI interpretation, differences between individuals are absolute. Thus, any difference between two taxa is an evolutionary difference. Moreover, polymorphism frequencies are irrelevant. More information is needed to determine the evolutionary relation of the taxa. Obviously, this interpretation will wreak havoc on PhCA as commonly applied, because evolutionary shifts will blur. For example, in the case of [3], if sister taxa differ in life history and mean of a climate niche trait, the difference in the latter may be attributed to the former, even if the climate trait is overlapping. In the PEI interpretation, the difference in life history cannot be attributed to the climate niche trait, because both life histories occur in the same climate niche. More information is needed to determine the relation between the variables, i.e., whether the life history transition coincided with climate niche shift, the overlap arising secondarily, or whether the transition occurred in the same climate niche, the mean niche difference arising secondarily.

$\mathrm{OE}$ [3] and Smith et al. [12] also appeared to assume that ambient climate niche traits analyzed are determined genetically and not epigenetically (including plasticity/elasticity). This assumption might be problematic in statistical analysis of climate niche based on empirical collecting locality data. Plants acclimate, and genetic components of acclimation plasticity vary at the population and species scales. Realized climate 
Hershkovitz

Montiaceae Ecological Evolution

incidence does not capture the entire climate niche breadth. Where a plant grows depends on many factors besides climate (e.g., substrate and competition). In fact, the collectively observed climate niche need not include the physiologically optimal conditions. The realized natural climate tolerances of a species reflect a compromise between climate traits and the myriad environmental factors. Also, climates change, generally radically and repeatedly at the mega-annual phylogenetic scale. Plant phenotype origin and/or subsequent incidence may reflect at least partially past and not current climates. Also, it is naïve to assume that climate traits at geographic localities and in the plant environment are the same or even functionally similar. As elaborated further below, climate parameters of the plant microenvironment may and often do differ from locality ambient means.

The specter of phylogenetic/genealogical uncertainty returns again in the species sampling strategy of [3, 12]. PhCA assumes that all analyzed operational taxonomic units are clades. This is because PhCA is a phylogenetic operation that, therefore, can be performed only on phylogenetic entities (clades and individuals). OE [3] and Smith et al. [12] recorded climate niche traits according to species (as identified in databases). Besides introducing the possibility for error due to misidentification and incomplete/underrepresented geographic ranges, this assumes that the operational species are monophyletic. But there is no evidence that Montiaceae operational species generally are monophyletic, nor, as implied above, that mean phenotypic values as determined from collection data are ancestral values. The species are type-referenced taxonomic entities (see [31]). As noted above, the PhCA also assumes that molecular marker gene trees and phenotypic trait trees are compatible.

The preceding assumptions in $[3,12]$ are technical. However, it must be reiterated that critical assumptions of PhCA applications are epistemological, and these affect the research in practical ways. Reviewing $[3,12]$ and PhCAs in general, it is important to avoid distractions of references to statistical/computational procedures and concepts, computer program packages, dazzling abstract graphics, burn-ins, number of millions of MCMC generations, and, most of all, alleged probabilities. More fundamental questions, such as those raised above and later in this discussion, cannot be answered by machines.

\section{PhCA of the pattern of temperature niche evolution}

OE [3] used locality climate data to assess taxon temperature niche. Two problems occur here. One is that the authors estimated species temperature niche as means calculated from collecting localities. But numerous Montiaceae species are widespread, a few, including naturalized populations, global in scale. Thus, as noted above, [3, 12] likely underestimated temperature niche evolution within species, hence within life histories. Moreover, especially for arctic/alpine plants [32], which include about 20\% of Montiaceae species, ambient air temperature departs considerably from plant environment temperature. Thus, some temperature niches estimated in [3] may be wildly inaccurate. 
Hershkovitz

Montiaceae Ecological Evolution

The PhCA found a marked increase in the rate of temperature niche diversification of Montiaceae relative to their outgroup. But they found no evidence for an abrupt shift into a different temperature niche regime. They attributed the expansion of Montiaceae into cooler environments to, effectively, stochastic phylogenetic drift (my term, not theirs). But the trend itself appears to be real.

Within Montiaceae, the trajectory towards cooler (and moister) habitats appears to elaborate with successive divergences. Phemerantheae are concentrated in relatively warm, arid environments in SW US and Mexico [33]. Subsequent diversification extended the range to the cool temperate habitats. Cistantheae origins presumably map to present day warm, arid northern Chile, with a secondary diversification in SW US (Calyptridium). But, although largely restricted to relatively low latitudes, many Cistantheae species are alpine. However, the historical ecological niche of modern Cistantheae species might be misleading. The lineage appears to be ancestrally arid-adapted, but it diverged before hyperaridization and the development of alpine habitats [3: Fig. 3; 4: Appendix S18, cf. 34, 35]

Among Montioideae, only Rumicastrum species are restricted to relatively warm environments [4]. However, this correlates with their restriction to Australia, which lacks the higher latitude/altitude extremes of other continents. Rumicastrum demarcates the basal split of Montioideae. All other Montioideae species concentrate at somewhat higher latitudes and/or altitudes. The exceptions prove the rule: the two technically tropical Calandrinia species are high elevation species. Hershkovitz [5] proposed that, following divergence of Rumicastrum, the ancestor of remaining Montioideae was situated in cooler, moister, and probably higher altitude southern Patagonia. Here would have originated South American Calandrinia, Antarctic Hectorelleae, and the ancestor of NW North American Montieae. A few descendants of Montieae extend (secondarily) into warmer arid S/SW North America, and then mainly into cooler, moister sites, whereas some Montiinae descendents are the only Montiaceae extended into in NE Siberia.

\section{PhCA of the pattern of life history evolution}

As noted, Montiaceae phylogeny manifests numerous transitions between annual and perennial life histories. OE [3] determined that, for Montiaceae and all of Portulacineae, the perennial species evolved from annual. In contrast, $\mathrm{OE}$ [3] determined that the annual species evolved from perennial. This is not an error. Different reconstructive methods yielded utterly contradictory results. The annual-to-perennial result emerged from a stochastic phylogenerative method. The perennial-to-annual result emerged from both maximum likelihood (ML)-based and parsimony phylogenetic reconstructive methods. OE [3] did not determine the source of the contradiction. They speculated that the annual-to-perennial result owed to inadequate statistical power of the method. They preferred the perennial-to-annual result because it was more "realistic." 
Hershkovitz

Montiaceae Ecological Evolution

OE [3] derived the perennial-to-annual pattern using a ML-based method (the "threshold method") that maps onto a phylogeny not the transition of the character state per se, but its "liability" of change, conditioned on the observed states of the tips. They demonstrated that, in the case of Montiaceae life history, the method yields results similar to that of maximum parsimony (MP) reconstruction. The difference is that MP reconstruction assigns discrete states to internal nodes. For example, in this case, MP reconstruction unambiguously assigned "perennial" to the base of the "Lewisia clade," (Lewisia + Lewisiopsis; monophyletic in [3] but not in [4]; see [5]) because all included taxa are perennials. The threshold method assigned to this node a small, but positive "liability" to evolve annuals, conditioned on the distribution of annuals in its outgroups.

Possibly not appreciated in this context is that the threshold method estimates evolutionary "tendency" (or its inverse, "constraint") as conceived by Wernham [36, 37]. Such inferred tendencies also appeal to the notion of orthogenesis, or "phylogenetic drive." In classical (intuitive) evolutionary taxonomy, such independently-derived traits were considered unobservable evidence of a tangible shared underlying characteristic, hence of phylogenetic relationship. Cantino [38] argued that such evidence could be introduced into formal MP cladistic inference, applying the term "apomorphic tendency," or underlying synapomorphy. But this notion is largely rejected for purposes of phylogenetic inference, because the origin of tendency is intangible. In particular, absence of trait does not signify absence of a tendency. One can assign the origin of trait tendencies to the progenote of all of life.

The threshold method, however, does not use liability to estimate phylogenetic relationship. Rather, it uses phylogenetic relationship to estimate liability of trait evolution, hence it estimates positive liability even in clades lacking corresponding traits. This is the same as statistical estimation of the origin of an apomorphic tendency. OE [3] noted that "liability is an unobserved, 'invisible' character, it may represent some other aspect of the organism's biology that would affect evolution of the observed discrete trait.” This paraphrases Cantino's [38] characterization. The method may have heuristic value in the search for such underlying traits, but otherwise it is merely a statistical implementation of Wernham's ideas and appeals no less to the notion of orthogenesis.

Encapsulated, the orthogenesis debate effectively is the same as the debate considering whether evolution is a first- or higher-order Markov process. In words, the former conceives evolution as a "random walk," not constrained by prior evolutionary steps. The latter means that evolution is predictably canalized by prior evolutionary steps. Earlier steps predictably canalize later ones. Thus, orthogenetic theory and the threshold method effectively interpret evolution as a higher-order Markov process. In this case, for example, the absence of annuals among species of Lewisia reflects chance failure to fully realize their inherent evolutionary tendency to evolve such. I return to the subject of orthogenesis in the context of chaos theory.

OE [3] emphasized annual versus perennial life history rather than growth form. Plant growth form comprises many and often ambiguous categories. As [3] noted, growth forms are difficult to diagnose. Indeed, among Montiaceae, life history appears to be a trait more discrete and accurately estimated than growth form. But the decision to analyze the former and not the latter seems to reflect at least partially analytical expedience. 
Hershkovitz

Montiaceae Ecological Evolution

1 Classification according to growth form increases the number of parametric categories, hence reduces effective

2 sample size, increases statistical variance, and reduces statistical power. It also introduces statistical complexity,

3 because it obligates derivation of an evolutionary transition matrix between all forms. This, in turn, assumes that

4 life forms are as objectively and consensually classified as are, e.g., nucleotides. Which is anything but true. But

5 even if growth form states behaved ideally, the statistical dilemma remains, because some growth form transitions

6 are rare among Montiaceae, e.g., involving rhizomatous, tuberous, or phanerophytic growth forms. Their

7 "transition probability" cannot be estimated with precision. Reducing growth forms to life history avoids this

8 complexity. But this is at the expense of blindness to these transitions and their implications vis-à-vis temperature 9 niche.

10 Reducing growth form to life history also homologizes life history transitions that are likely not

11 homologous. Several perennial Montiaceae are rosettiform-caudiciform hemicryptophytes that produce annually

12 relatively little and ephemeral shoot tissue. Some species are essentially acaulescent, producing nothing atop the caudex apex but a crop of leaves and associated inflorescence branches. OE [3] idealized this extreme as the quintessential Montiaceae perennial. Phylogenetic reconstruction has suggested that rosettiform-caudiciform is the ancestral growth form of Montiaceae [3] (but see below). O'Quinn [39] studied the anatomy of perennial species of Claytonia and determined that the subterranean perennating structure of tap-rooted species is a caudex, i.e., comprises a persistent radicle, hypocotyl and base of the epicotyl. Quite likely this applies to all tap-rooted perennial Montiaceae. Larger plants of several genera have multiple crowns arising from a branched caudex.

But not all Montiaceae perennials are of the rosettiform-caudiciform sort. Tuberous forms of Claytonia and presumably also Phemeranthus are caudex derivatives. O'Quinn [39] demonstrated that, tubers of perennial Claytonia species are essentially the epicotyledonous portion of a caudex, or a caudex lacking a perennial taproot, i.e., caudex-derived tubers. Other perennial Claytonia species have rhizomes, stolons, and bulbilliferous stolons. The sister-genus Montia, comprises mainly perennial species, none caudiciform. All are stoloniferous and one forms stolon bulbils. Non-caudiciform perennials also occur among other Montiaceae (Lewisia, Calandrinia, Rumicastrum, Cistanthe. and Lenzia; see below).

$\mathrm{OE}$ [3] also equated Montiaceae life history evolutionary lability with the capacity to shift between above-ground and below-ground mass allocation. It is to this effect that they idealized the Montiaceae perennial form as an acaulescent tap-rooted caudiciform whose above-ground mass is low and ephemeral. This morphology characterizes, e.g., many but not all species of Calandrinia sect. Acaules and Lewisia. But, as noted above, this mischaracterizes noncaudiciform Montiaceae perennials and even some caudiciform species. Some caudiciform perennial species of Calandrinia, Cistanthe, Lewisia, and Montiopsis evidently allocate massively to substantial above-ground structures. In the cases of Lewisia sect. Cotyledon leaves and Calandrinia caespitosa mat ramifications, these structures are not ephemeral. It is not clear that above-ground mass allocation in these 
Hershkovitz

Montiaceae Ecological Evolution

caudiciform taxa is proportionally less than in the more diminutive annuals. In fact, [3] provided no data on above/below-ground mass allocation, hence their speculation on its evolutionary significance is not evidential.

In summary, in order to qualify as a scientific method that proves something, PhCA seems to demand a preference for naïve interpretation of phenotypes as a more coin-like rather than a more complex dice-like or bingo ball-like parameter. But which categorization captures more biological information? Life history or growth form, which includes life history? The fundamental problem of growth form classification pertains to homology. Growth form classifications are typological. Different classifications classify similar forms hierarchically differently. As is the case with all typological morphological, taxonomic, and ecological classifications (e.g., "fruit types," species, biomes), often entities found in nature intergrade and resist classification. Moreover, classifications prejudice interpretation and/or obscure ontological relations between forms.

Besides reducing the number of parametric categories to two, [3] also explicitly preferred life history analysis because plants species usually are unambiguously classified and not polymorphic for this trait. However, the reduction of growth form to life history does not overcome ambiguity. As [3] noted, life history of a few Montiaceae species appears to be developmentally plastic (as discussed later), and a few bear either vestigial or rudimentary traits of alternate life histories. A few are ephemeral but effectively iteroparous, and others are perennial but with characteristics of semelparity (see below). Towards the objective of evolutionary explanation, these infraspecific "in the act" examples provide valuable clues [40] (see below). PhCA predicates to explain evolution, but statistical expedience discards evidence of evolution in the act in favor of interpretation millions of years after the exculpatory evidence has sublimed. Furthermore, as detailed below, ecological shifts emphasized by [3] have been achieved in some cases by change in growth form but not life history. Equating life history transitions involving different growth forms can be problematic.

Clarification of the denomination "annual" underscores the difficulty in classifying even an apparently coin-like character. Annual is a term applied to therophytes, which complete a monocarpic (semelparous) life history during the course of a physiologically favorable period within a single year. The annually repeating cycle is important from an ecological perspective, because the ecological proactivity of annual species fluctuates markedly during the course of a year and, especially in the case of several Montiaceae, year-to-year. But from a developmental standpoint, the duration of the growth period is arbitrary. Many monocarpic (semelparous) species persist for two to more than 100 growing seasons (e.g., Agave and Bambusa spp.), but they are not perennials in the same way as polycarpic (iteroparous) species. At the same time, some polycarpic species are ephemeral, behaving as annuals in seasonal climates but persisting several years in more uniform climates.

Most Montiaceae annuals appear to be of the monocarpic therophyte sort, but, as [3] noted, classification of some species is uncertain (see also below). I am not aware of studies measuring the maximum lifespan of nominal Montiaceae annual species under physiologically ideal conditions. For example, the genus Montia includes (nominal) annuals and stoloniferous perennials, the latter perennating via ramets formed at rooting 
Hershkovitz

Montiaceae Ecological Evolution

nodes. But two nominally annual species nodal adventitious roots and behave clonally. They are described as annual, not because they are monocarpic, but because they appear to be more ephemeral than other species.

Some Montiaceae perennials also may behave physiologically and ecologically as annuals. In particular, like many geophytes, the tuberous species of Claytonia can be described as "perennial therophytes" in that the above-ground growth is short-lived and dies following flowering. The tuber is developmentally seed-like, especially because of the well-known cytological instability in this group (11). Interestingly, many Montiaceae perennials have deciduous above-ground growth. Among Montiaceae, exceptions include the evergreen species of Lewisia sect. Cotyledon and, at least in cultivation, Lewisiopsis tweedyi [41, 42], and two phanerophytic species of Cistanthe (see below). Thus, life history might seem to be the most coin-like of traits among Montiaceae, i.e., they are either "heads" or "tails." But scrutiny reveals shades of gray: from polycarpic annuals to effectively monocarpic perennials to long-lived phanerophytic perennials. Ecologically, these are not all equivalent.

$\mathrm{OE}$ [3] attributed the "elevated lability in life history strategy [of Montiaceae] relative to other Portulacineae....to an increased flexibility in biomass allocation." Descriptively, this is accurate (though teleological), but trivial. All of development is differential biomass allocation. So lability of life history "strategy" is synonymous with flexibility of biomass allocation....strategy. Whether programmed or induced, any developmental shift is accompanied by a corresponding shift in biomass allocation. Whether one explains the other is unknown, hence a chicken-and-egg question. From a physiological standpoint, OE [3] presented no evidence that Montiaceae allocate biomass physiologically differently than other Portulacineae or other plants generally. Ultimately, the question is whether Montiaceae bear some characteristic that is causal to their realized life history and growth form lability.

Montiaceae life history and growth form evolution appears to involve homeosis, especially paedomorphosis. In particular, the perennial acaulescent form is essentially an oversized and long-lived germling. The perennating bulk of the plant is mainly radicle, hypocotyl, and a short epicotyl [39]. In many acaulescent Montiaceae, the leaves are ephemeral. One might suppose that much of the lateral root system is also, as this would not be expected to survive the extreme drought or cold conditions. In any case, the "juvenility" of the acaulescent form may relate to its evolutionary lability in life history/form. But this does not identify the causality of Montiaceae life history/form evolution. Acaulescence/hemicryptophyty is neither necessary (e.g., Portulaca), nor sufficient (e.g., Phemeranthus, Anacampserotaceae) to provoke the evolution of an annual life history.

\section{PhCA of the relation of temperature niche and life history transitions}

OE's [3] analysis purported to demonstrate a phylogenetic correlation between annual/perennial life histories and, respectively, warmer/cooler temperature niche. This is to say that the correlation of transition between life history and temperature niche, as circumscribed and recorded, was statistically significant and 
phylogenetically independent, and that its directionality was shared in most (but not all) instances. The authors noted that the phylogenetic correlation is shared in other herbaceous lineages diversified in western America, corroborating this "lawful" relation. Nuances discussed in preceding sections underscore the challenge of such reductionist analyses of macroevolution. For example, as noted, [3] recognized the difficulty of classification according to growth form and preferred analysis according to more discrete life histories. This ostensibly rendered classification simpler and less ambiguous, but also provided larger class sizes for statistical analyses. They concluded that annuals and perennials are adapted to, respectively, warmer and cooler environments.

More precisely, [3] concluded that ancestral Montiaceae were hemicryptophytic slow-growing perennials with substantial below-ground biomass and more limited and ephemeral annual above-ground growth. They associated these traits with colder environments, which would inhibit both growth rate and growing season, rendering maladaptive annual life histories (see below). Annuals, they concluded, evolved multiple times in response to evolution into warmer environments, in particular those with marked precipitation seasonality that thus facilitated more rapid growth, hence higher seedling survival, and likewise greater seed production. They argued that the frequency of this life history transition reflected the ease with which this herbaceous growth form could reallocate mass from below-ground to above-ground growth. OE [3] explained the infrequency of annuals in cooler environments (high latitudes/altitudes) as a consequence not of temperature, per se, but of growing season length. They argued that the growing season at high latitudes/altitudes is too short for annuals to complete their life cycle.

OE [3] did not explore more direct possible temperature effects, such as subfreezing summer night temperatures in the alpine zone. Caudiciform and other specialized growth forms and/or physiologies protect the plants (and their reproductive buds) from freezing, but annuals would tend to be exposed and not have a caudex full of solutes to pump antifreeze. Presumably, such protections come at the price of reproductive effort. However, some advantages of alpine perennials in the alpine zone may be liabilities in warmer environments. These may include physiological traits. In fact, many alpine species are notoriously difficult to cultivate in warmer environments. They rot.

OE [3] also did not dwell on possible secondary consequences of temperature that might underwrite success in warm environments and disfavor them in cooler ones. Warm, arid zones, especially in Chile, are open territories. Even in Chile's semidesert region, vast tracts of fine substrate lay bare most of the year and for several years continuously during regular droughts. Cooler regions are dominated by perennial vegetation that naturally resists annual invasion, especially in shrubby and forested habitats. But even in the herbaceous alpine zone, mat forming and/or rhizomatous perennials tend to monopolize available sites otherwise suitable for annual herbs. Thus, the effect of temperature on life history might be partially indirect, consequent to competition.

Substrate might be a factor, as well. The warm, arid zone has tracts of flat, fine, well-drained substrate, which make excellent flower beds for annuals. Substrate in the alpine zone tends to be rocky and steeply sloped. 
Hershkovitz

Montiaceae Ecological Evolution

Neither is optimal for short, fibrous annual root systems. Flatter substrates often are muddy or boggy owing to prolonged runoff from snowmelt.

None of the preceding negates the hypothesis of [3]. Rather, the misquoted "proof is in the pudding" applies. The plausibility of [3] requires critical evaluation of the phylogenetic and ecological evidence. Before proceeding with an analysis of Montiaceae, broader evidence for the plausibility of this scenario should be examined. Plant life history evolutionary direction and cause have been questions long debated in evolutionary biology [40, 43]. Prior to the development of molecular phylogenetics, the perennial form was presumed by default as ancestral. This may have reflected a precladistic and essentially Aristotelian notion of which angiosperm traits were inherently primitive and which were advanced. Prior to the molecular phylogenetic revolution, this notion was deeply and perhaps intransigently entrenched in narrative evolutionary interpretation, even of Portulacineae [e.g., 44]. Prior to [3], proof of derived perenniality had emerged in phylogenetic analyses of different lineages [e.g., 43, 45], including Portulaca [7] and Calyptridium [46, 47]. The polarity, however, does not negate a particular causal argument, as long as the causal factor also operates reversibly.

Multiple factors have been identified as possible evolutionary drivers of life history transitions. These include the climatic factors emphasized in [3]. Others include polyploidy, breeding system, and pollinating agents $[10,43,45]$. Ultimately, the question boils down to the survival adequacy of different life histories. The Darwinian adaptationist paradigm frames the question in terms of fitness. Perennials are presumed to invest more resources toward persistence, and annuals towards reproduction. Depending on the taxon in question and conditions, there is a point at which one life history becomes more fit than the other, facilitating transition. And presumably there are extremes where one is fit and the other not, obligating transition. OE [3] seemed to emphasize extremes, highlighting environments where annuals/perennials approach, respectively, 0\%/100\% incidence. This demarcated a temperature gradient, which they argued was the explanation. The present work rejects statistical interpretation, as elaborate later, and emphasizes adequacy rather than necessity of conditions and absolute rather relative fitness. Thus, the present work adopts a criterion of necessity/sufficiency of explanation.

Does cooler/warmer temperature provide a necessary and sufficient explanation for transitions between, respectively, perennials and annuals? OE [3] emphasized corroborating examples from other lineages, but did not elaborate as well counterexamples. Although [3] recognized that some annuals are alpine species, possibly they did not appreciate that the central/southern Andes harbor exceptionally many annuals [48.], and they did not note the five examples among Montiaceae (see below). And, more generally, both warm and cold environments, including arid and/or seasonally/episodically moistened ones, harbor many if not mainly perennials. While warm and arid environments harbor much annual diversity, warm, humid environments harbor exceptionally little. Thus, temperature alone does not pass the necessity/sufficiency test. 
Hershkovitz

Montiaceae Ecological Evolution

Even warm, arid environments, known for their higher proportion of annuals, including those blessed with Montiaceae, include herbaceous perennials, especially geophytes, i.e., plants with massive below-ground and ephemeral above-ground growth. Of special significance are taxa diversified into both colder and warmer habitats in the same region as Montiaceae, e.g., Chile and adjacent Andean territories. In these, there are numerous exceptions to the temperature/life history "law." As noted above, this region has exceptional annual diversity in the alpine zone, even if the balance favors the warm, arid zone. An example is Chaetanthera s. 1. [49], which includes, among others, a clade of five altiplano annuals sister to a sympatric clade of caudiciform hemicryptophyte perennials.

The central/southern Andean region harbors high Oxalis diversity, mostly perennials, with fewer annuals arising sporadically [34]. While the annual species indeed occur at lower elevation than most of their perennial counterparts, numerous perennials are sympatric with them at low elevation, Several are warm/arid-zone species, including a sizeable clade that includes the most sizeable phanerophytic species. Alpine species are caudiciform hemicryptophytes. These presented numerous unrealized opportunities to sire radiations of warm/arid annuals.

As [3] noted, only two other Portulacineae lineages are primarily herbaceous, Portulaca (Portulacaceae) and Anacampseros (Anacampserotaceae), and both include caudiciform species. Most species of Portulaca are annual. Being C4 plants, Portulaca is distributed in hotter and more tropical environments. But since Portulaca is closely related to other warm, arid adapted taxa, it is difficult to conclude that the annual life history evolved in response to warm habitat temperature. Most perennial species usually occur at higher elevations than their nearby annual relatives [7], supporting this correlation proposed in [3]. But two (?) annual species, especially (one or more forms of the evidently polyphyletic; [7]) P. oleracea, have spread well into the cool temperate zone and are considered invasive. $\mathrm{C} 4$ plants require adequate light and warmth to survive. $\mathrm{C} 4$ perennials generally do not survive at cool, high latitudes, but many C4 annuals do. Thus, among Portulacaceae (s. str.), it appears that the annual life history allows plants to survive colder environments with shorter growing seasons-exactly the opposite of what [3] proposed for closely related Montiaceae.

Anacampserotaceae includes succulent caudiciform hemicryptophytes, some essentially acaulescent, of warm, arid environments [8, 9]. But no annual species evolved. Except for the woody scrambler Grahamia, the species are slow-growing, perhaps related to their CAM photosynthesis. Although they evolved a growth form similar to some Montiaceae species, for physiological and developmental reasons, possibly they evolved themselves into a dead-end in terms of life history evolution. The same may be true of their phytogeographic compatriots and more distant cousins, the highly diversified Mesembryanthemaceae, which likewise includes many succulent acaulescent caudiciform hemicryptophytes and cryptophytes. Again, no annuals evolved, even though their environment is warm and arid.

Thus, out of the gate, the evolutionary scenario of [3] presented inadequately addressed nuances undermining its plausibility. Some angiosperm taxa might corroborate the scenario, but others do not, including 
Hershkovitz

Montiaceae Ecological Evolution

other Portulacineae and morphologically/ecologically similar Caryophyllales. Even though life history seems correlated with habitat temperature, high temperature lacks necessity/sufficiency to induce a transition to annuals, and cold temperature lacks necessity/sufficiency to induce transition to perennials. The exceptions to the temperature "rule" might be explained by taxon-specific circumstances, such as C4 photosynthesis. But that is the point of PEI. All taxa have taxa-specific circumstances. Still, the evidence does not negate the plausibility of the scenario of [3] for Montiaceae.

But, as elaborated in greater detail in the narrative reinterpretation below, additional evidence not mentioned/explained by [3] compromised the a priori and a posteriori plausibility of the elaborated scenario. For example, O'Quinn [39] suggested that the caudex-derived tubers of Claytonia sect. Claytonia species adapted them to warmer environments -- the same as OE [3] suggested for annuals. The tuberous forms complete their above-ground phase faster and with less growth than the deep-rooted caudiciform species, which are restricted to the coldest environments. Is it possible, therefore, that, in flagrant violation of the "law," a transition from annual to perennial can adapt plants to a warmer environment? As discussed below, yes.

In the meantime, the observation of [39] might suggest that ephemerality of organs rather than individuals (i.e., life history) adapted Montiaceae to warmer environments. But the reverse also has occurred. The species of Lewisia and Cistanthe with persistent above-ground organs occur in warmer environments than species with ephemeral above-ground organs (see below). Thus, reducing diversity to artificial phenotypic classes, however valuable heuristically, discards considerable evolutionary evidence and might mislead evolutionary analysis.

OE [3] explained the absence of annuals in the alpine zone as consequent to its shorter growing season. But many Montiaceae annuals occur in hyperarid deserts and, as [3] alluded, the effective growing season is extremely short -- shorter, in fact, than in the alpine zone. More importantly, the hypothesis is falsified directly by the occurrence of any annuals at high elevations. Two Montiopsis annuals are restricted to high elevations, and another extends to the alpine vegetation limit [50]. Calyptridium pygmaeum and Philippiamra minuscula (Cistantheae-Calyptridinae) are alpine [51, 52]. Finally, the growing season hypothesis does not explain the restriction of many Montiaceae perennial species to the alpine zone. Is the growing season at lower altitudes too long? These and other observations collectively indicate that, even if the statistical approach in [3] is epistemologically validated, the conclusions were counterevidenced a priori.

\section{Narrative re-interpretation of temperature niche and life history evolution among Montiaceae}

To summarize the preceding, global reconstructive analyses undertaken thus far $[3,6,8]$ indicate that Montiaceae ancestrally were perennial. This is the life history of the successive outgroups, as well as Phemerantheae, the outgroup of all other Montiaceae. The ancestral growth would appear to reconstruct as a rosettiform-caudiciform hemicryptophyte, the form characteristic of many Phemeranthus species [33] and many 
Hershkovitz

Montiaceae Ecological Evolution

1 perennial Cistantheae species. Successively diverged Montioideae (Rumicastrum, Calandrinia, Hectorelleae,

2 Montieae, and Montiinae) also have species that share this form. Other perennial growth forms also occur,

3 including rhizomatous (Lenzia, several Montiinae), tuberous (Claytonia, Lewisia), and pachycaul forms that are

4 chamaephytic to phanerophytic (Cistanthe). Reconstructive methods have suggested that annual species of

5 Montiaceae generally evolved independently many times from perennial. PhCA methods [3] indicate that the

6 annual-perennial transition correlates with temperature niche difference, implying a causal relation between the 7 two. hard to document definitively. Support for reconstructed life history evolution among Montiinae seems to base

The following narrative analysis summarizes evidence for numerous instances of the evolution of Montiaceae perennials from annuals. I show that instances of the more "realistic" reverse polarity are remarkably partially in unfounded assumptions imposed over yet-to-be-resolved phylogenetic nodes. I also argue that the inferred relationship between life history and temperature niche is more coincidence than a true causal relation. Support for the latter inference owes to, probably among other factors: missing data and/or inadequate phylogenetic resolution pertinent to several alpine annual species, discarding evidence for ecological diversification among perennials, and unjustified extrapolation of rates of life history evolution from those of DNA sequence evolution. There is also an element of phylogenetic chance....extant taxa document some differential frequencies of transitions that do not necessarily reflect their potential to occur.

\section{Phemerantheae}

Phemerantheae all are caudiciform hemicryptophytes and include the monotypic altiplano genus Schreiteria and the diverse genus Phemeranthus. Approximate locality data from the former suggests that it is adapted to fairly warm, subhumid summer conditions and cool winters. Species of the latter range into the cool temperate zone and one occurs in the altiplano. But most species occur in the semiarid S/SW US and Mexico, where winters might be cool, but summers are exceedingly hot. According to [33], some species of Phemeranthus are "legendary" for their capacity to flower under conditions of extreme heat and drought.

Thus, Phemerantheae provide examples of warm-adapted caudiciform hemicryptophytic Montiaceae, as well as Montiaceae of this form that diversified across temperature ranges without changing life history or even life form. There are caveats. Phemerantheae are the trans-cordilleran sister to cis-cordilleran Montiaceae. The trans-cordilleran climate has year-round, but especially summer precipitation and humidity. The cis-cordilleran climate has mainly winter precipitation and, in the arid zone, this can be scarce or absent for years at a time. Thus, from a statistical standpoint, these are apples and oranges.

Also, it is possible that the physical origin of life history lability of Montiaceae maps to the origin of cismontane clade. A caveat in this case is that, at the time of this origin in the latest Eocene or earliest Oligocene, the 
Hershkovitz

Montiaceae Ecological Evolution

physical and climatic conditions across this region were more uniform. The differentiation originated millions of years later. The modern conditions did not mature until perhaps latest Miocene or Pliocene [34]. And all genetic evidence indicates that most Montiaceae life history transitions occurred after that.

However, from the dogmatic statistical PhCA adaptationist standpoint, these observations provide, at best, "excuses" for why organisms do not behave as PhCA conclusions would oblige them. To wit, [3] generalizes that all Montiaceae have an evolved life history lability. Thus, all Montiaceae should manifest this. They generalize that annuals/perennials occupy warmer/cooler niches, and corresponding transitions occur, and thus ought to occur, in response to corresponding temperature changes/differences. This has not happened. The very notion of particularizing exceptions betrays not only the fallacy of the generalization, but the method used to derive it and its rationale. However, these shortcomings do not discredit the value of PhCA as a heuristic tool and in generating statistical systematic descriptions. Systematic descriptions, statistical or narrative, always have served a heuristic purpose.

\section{Cistantheae}

All polytypic Cistantheae genera are polymorphic for life history. Reconstructive methods indicate that the ancestral form was perennial, presumably a rosettiform-caudiciform hemicryptophyte. But in some cases, the perennial form seems to have been derived from annual, as elaborated below. This evidence along with a small degree of phylogenetic uncertainty renders at least questionable the life history of the ancestor.

Montiopsis. The rosettiform acaulescent perennial form characterizes (more or less) only one species of Montiopsis, M. (subg. Dianthoideae) cistiflora. All other perennial species have more elaborated and leafy stem systems. Some perennial species of M. subg. Montiopsis produce considerable above-ground growth [50]. With their woody, branched caudex, these species might be considered "hemi-suffruticose."

Reconstruction suggests that the ancestral form of Montiopsis was perennial. Phylogenetic resolution among Montiopsis subg. Montiopsis is inadequate to evaluate life history evolution in this subgenus. But all four species of Montiopsis subg. Dianthoideae are perennial. Moreover, their pollen is tricolpate, whereas all $M$. subg. Montiopsis share the presumably derived pantoporate morphology. The outgroup criterion would favor a perennial ancestor in subg. Montiopsis, but the high divergence separating Montiopsis from other Cistantheae and extremely low genetic divergences between annual and perennial species renders both logically and "statistically" possible an annual ancestor (see below).

Another caveat in interpretation of Montiopsis life history evolution a propos Cistantheae is certainty with respect to its inclusion in this clade [5]. In any case, its molecular divergence from other Cistantheae is rather high. These caveats create two possibilities. One is that Montiopsis does not belong in Cistantheae, hence life history evolution of the latter is irrelevant. This possibility is remote [5]. The other is that the long branch 
Hershkovitz

Montiaceae Ecological Evolution

separating this genus introduces inherent ambiguity with respect to its ancestral phenotype, analogous to the scenario of Oriastrum (Chaetanthera s. l., Asteraceae; [49]). Thus, the growth form and life history of modern $M$. subg. Dianthoideae might be consequent not to the original form of the lineage, but to the modern endemism to the recently developed alpine zone. By such scenario, it is possible that annual species of $M$. subg. Montiopsis retain an ancestral form. The long branch and life history polymorphism among other Cistantheae render this question unresolved.

Only annual species of $M$. subg. Montiopsis occur in the warmest portion of the generic distribution, i.e., low elevations of the Atacama Desert. This accords with the temperature niche correlation described by [3]. But closer inspection of distributions [50] reveals significant departures from the ecogeographic interpretation of [3]. Two of the nine annual species are alpine species: M. glomerata and M. modesta. Another, M. cumingii, is montane to alpine/altiplanic. Another, M. berteroana, which defines the southern cooler end of the annual distribution, extends from low elevations to nearly the alpine vegetation limit in that zone. Incidentally, the alpine growing season at this latitude (ca. 38S) is markedly shorter than that of most Chilean annual Montiaceae species, which occur mainly between 25-35S. Meanwhile, five of the six perennial species indeed are strictly alpine, but one, $M$. umbellata, has a range essentially completely sympatric with the annual M. berteroana, which with it does not seem to be especially closely related.

The significance of Montiopsis towards understanding of life history evolution among Montiaceae is thus two-fold. For one, it provides examples of species that buck the statistical trend. Four of nine annual species are restricted to or range into the alpine, and one of six perennial species ranges to lowest elevations. Secondly, the very low genetic divergence among species of $M$. subg. Montiopsis may be problematic for corroborating life history evolution, but it is not uninformative. It demonstrates that little divergence and little time is required to evolve from one morphological and, independently, ecological extreme to the other. The perennial species include robust plants with showy flowers, while the annuals include diminutive plants with inconspicuous flowers. This phenomenon reemerges in the analysis of other taxa below.

Cistanthe. Annuals and perennials occur in both sections of Cistanthe. Phylogenetic resolution is inadequate to pinpoint most evolutionary transitions. Cistanthe sect. Cistanthe include at least one annual species and another that might be facultatively annual [1]. The section is unique among South American Montiaceae in that it includes mostly low elevation perennial species: C. aegitalis, C. crassifolia, $C$. grandiflora, (incl. $C$. glauca; [1]) C. laxiflora, C. mucronulata, and probably others whose indentify remains tentative including $C$. speciosa [1]. The only high elevation perennial species are $C$. discolor and C. cabrerae. Perennial growth form in $C$. sect. Cistanthe ranges from caudiciform to phanerophytic pachycaul shrubs. But above-ground growth in the more caudiciform taxa is substantial. Ironically, the most "acaulescent" species is the hemicryptophytic annual $[1]$. 
Hershkovitz

Montiaceae Ecological Evolution

The perennial life history in Cistanthe sect. Cistanthe itself does not appear to correlate strongly with climate. Two perennial species are high elevation, but the remaining are lowland. Most occur in relatively cooler coastal habitats of the otherwise warm, arid zone, but some occur in or extend into warm to hot interior valleys [1]. Cistanthe crassifolia is notable, because some individuals grow into a sizeable pachycaul shrub commonly 1$2 \mathrm{~m}$ in height and breadth. It is endemic to dunes of the fog-laden coast north of Huasco Province (Region III), hence its size elaboration may indeed owe to cooler temperature. However, interior desert valley forms pertaining to this section, while chamaephytic, also attain considerable size. In C. grandiflora and sometimes in other species, the annual above-ground growth attains 1-2 meters in height, hence challenge the simplistic typological notion of "hemicryptophyte." Some plants attain two meters in height. The annual species [1] occurs usually towards or at the cooler coast environment of the warmer central latitudes of the sectional distribution.

The ecological pattern in $C$. sect. Rosulatae conforms partially to OEs [3] inferred correlation between life history and temperature niche. But there is no phylogenetic evidence that the annuals are derived from perennials. To the contrary, evidence suggests the reverse in most cases. All but six of the species of $C$. sect. Rosulatae subsect. Rosulatae [1] are annual, and these generally occur in warmer environments than the perennials. Four of the six perennial species are alpine plants at one time classified in Reiche's Calandrinia sect. Andinae [1]. Existing evidence does not resolve the phylogenetic position of these taxa within C. sect. Rosulatae subsect. Rosulatae. But, given that successive outgroups of $C$. sect Rosulatae among Montiaceae and Portulacineae are mainly warm-adapted lowland taxa, there is no a priori reason to presume that the temperate alpine habitat of Andinae species is ancestral among $C$. sect. Rosulatae subsect. Rosulatae. It is not impossible, but improbable, given morphological specializations of these species and recent origin of their alpine habitat.

The annual species of the Andinae affinity, $C$. oblongifolia, indeed occurs at elevations below that of $C$. humilis. However, the two species hybridize and the hybrids produce pollen and seeds. It seems reasonable to assume that $C$. oblongifolia and $C$. humilis are either sister or progenitor-descendent species. Phylogenetic data are inadequate to resolve this question. Nonetheless, evidence for hybridization underscores the weakness of the assumption of PhCA methods that evolution is strictly cladogenetic.

Other presumably perennial species of $C$. sect. Rosulatae subsect. Rosulatae belong to the historically misunderstood C. arenaria complex, which has the southernmost distribution of all of Cistanthe [1]. These resemble the annual species misidentified as C. arenaria of the warmer and more arid provinces further north [1]. Members of the perennial C. arenaria complex range from low valleys (ca. $550 \mathrm{~m}$ ) of the high cordillera (ca. $3000 \mathrm{~m}$ ) to warm lowlands of the central valleys, where summer high temperatures commonly exceed $30 \mathrm{C}$, to cooler fluvial sands along the coast. The interior forms are decidedly suffruticose at the base. The maritime form is more succulent and completely herbaceous.

As noted, existing molecular data do not resolve relations among species of $C$. sect. Rosulatae. But the outlying distribution of the $C$. arenaria complex suggest that it is derived within the section. Hence, their 
Hershkovitz

Montiaceae Ecological Evolution

1 perennial life history would be derived and temperature niche would be derived also. The former is contrary to

2 [3], but the latter is in agreement with the correlation between life history and temperature niche. At the same time, it must be appreciated that temperature niche is broad in this complex, ranging from cooler montane to warmer lowland to cooler maritime environments. Here, there is no shift in life history, although the evolutionary temperature niche trajectory in the complex is unknown. Being more herbaceous, it might seem that the coastal forms are developmentally "closer" to becoming an annual, but this is speculation.

The final perennial species of the section is $C$. guadalupensis, a succulent pachycaul shrub endemic to Guadalupe Island, off the coast of Baja Mexico. Phylogenetic evidence indicates that it is derived from an annual growth form. Cistanthe guadalupensis is the only perennial species of $C$. sect. Rosulatae subsect. Thyrsoidae, a clade of six species [1]. Phylogenetic relations among the species remain unresolved. A sister or even a progenitor-descendent relation with relatively nearby $C$. maritima (coastal northern Baja California to southern California) seems most likely on morphological and phylogeographic grounds. The inflorescences of both species have fewer and much larger flowers than other species of the subsection, and independent origins obligate two LDD events from South America [cf. 5]. Distribution of these species suggests similar climate niche. However, winter mean high temperatures of Guadalupe I. are somewhat warmer than those of most of the range of continental C. maritima.

Thus, the molecular data demonstrate something that evidently OE [3] did not anticipate. Montiaceae are a clade of mostly cooler-adapted hemicryptophytic perennials and mostly warmer-adapted (but still temperate zone) annuals. OE [3] thus detected a statistical correlation between corresponding evolutionary transitions of life history and temperature niche. But Cistanthe guadalupensis is a relatively warmer-adapted pachycaul shrub -similar to the Namibian Desert Portulacaria armiana (Portulacineae; Didiereaceae) -- but derived from an annual 22 herb.

This is just another example of manifest evidence that statistical correlations in ecology/evolution are not ecological/evolutionary (predictive) rules. As it happens, modern Montiaceae happen to include a number of annual-perennial and warm-cold transitions sufficient to "prove" a correlation. But this reflects partially the number of Montiaceae lineages that happen to exist and the geography of where most happen to occur. But the correlation is a "prediction" only in hindsight. Had historical circumstances resulted in more present day $C$. maritima-guadalupensis and C. crassifolia perennial examples, more alpine annuals, and fewer lowland annuals examples, the conclusions in [3] would have been less strongly or not at all statistically supported. This theme will resurface repeatedly in this discussion and will be summarized in the conclusion. taxa separated by many millions of years of divergence, viz., morphological/ecological divergence covaries with molecular divergence. Some PhCA methods extrapolate rates of phenotypic evolution according to phenotypes manifested among phylogenetic terminals. Phenotypic evolution is not necessarily directly scaled to molecular 
Hershkovitz

Montiaceae Ecological Evolution

1 phylogenetic branch lengths. As in [3], stochastic transition probabilities are conditioned on time-calibrated

2 molecular phylogenies. But the effect is similar. The phenotypic divergence between C. guadalupensis and other

3 species of its subsection is comparable to that between Cistanthe and Portulacaria, separated by about 50 million

4 years [3]. Were it not for the intervening taxa, PhCA methods would infer a remarkably slow rate of growth form

5 evolution. Yet the form of $C$. guadalupensis evolved -- in terms of geological time -- very rapidly from a

6 therophyte.

In summary, the only trait of the perennial species of Cistanthe that reconstructs as ancestral appears to be perennation itself [3: Fig. $5 c$ ]. But this reconstruction rests partially on lack of phylogenetic data and/or resolution. No other morphological traits seem to support independently perenniality of subclade ancestors. Thus, regardless of the ancestral state, I presented here evidence that additional phylogenetic resolution will show perennation to be derived multiple times in this section.

The genus as a whole might show a correlation but no fixed and predictable relation between life history and temperature niche. Perennial species of $C$. sect. Cistanthe span the entire temperature niche breadth of the section, and the annual form occupies an intermediate temperature niche. Most perennial species of $C$. sect. Rosulatae indeed occur at the colder end of the sectional temperature niche spectrum, but $C$. guadalupensis is a notable exception.

Calyptridinae. The ancestral life history reconstructs as perennial [3: Fig. 5c], but this possibly reflects inertia from reconstructions in the other genera, which I consider questionable (see above). The perennial taxon Lenzia is sister to the remainder of Calyptridinae, but its morphology is peculiar to this genus [1]. Its form of perennation remains subject to analysis, but I regard it as rhizomatous, hence not caudiciform as in other perennial Cistantheae. From its morphology [1], evidently extremely infrequent flowering, and distribution, I speculate here that ancestrally it was a mat-forming species that now forms only loosely associated patches of individuals originating rhizomatously. Overall, the morphological, geographical, ecological, and molecular divergence of Lenzia renders somewhat likely that its perennation is not homologous to that in other Cistantheae.

Ecologically, the perennial species of Calyptridinae accord with the correlation between life history and temperature niche, but alpine annuals also occur. Lenzia is an alpine taxon, and both perennial species, of Calyptridium, C. monospermum and C. umbellatum, occur at higher elevations than most of the annual species. Calyptridium monospermum is montane to subalpine, while $C$. umbellatum is subalpine to alpine. Perhaps correspondingly, C. monospermum evidently is shorter-lived. Its caudex is slender, and, in populations I have visited, most flowering individuals indeed appear to be current season germlings. Possibly it behaves facultatively as a winter annual. I have never seen an individual of "true" C. monospermum with a truly "old" caudex. The largest caudices I have seen in this species occur in zones of hybridization with C. umbellatum [53]. 
Hershkovitz

Montiaceae Ecological Evolution

1 Guilliams et al. [55] suggested that the latter taxon might behave as a biennial ("winter annual"). If so, possibly it

2 represents incipient evolution of a caudiciform perennial. Given that it is a high-elevation species, possibly it

3 documents the correlation between life history and temperature niche inferred by [3]. Similarly, Claytonia rubra

4 subsp. rubra also is reported to behave as a facultative winter annual (see below).

If Calyptridium parryi var. martirense and Claytonia rubra subsp. rubra indeed behave as facultative winter annuals in cold environments, they may provide evidence of life history evolution via plasticity-mediated genetic assimilation [56]. Presumably, this behavior in cold environments is a plastic response reflecting developmental genetic temperature sensitivity of inflorescence/floral development [57-59]. In colder environments, unrelated physiological mutations may occur that render obligatory what had been a plastic vernalization response, i.e., developmental delay at low temperature. In colder environments, the facultative and obligate vernalization phenotypes would be identical. Their distinction would manifest only in warm environments, where the obligate vernalization phenotype would be maladaptive. Thus, the obligate vernalization phenotype would be restricted to colder environments. They would be obligate winter annuals.

Survival of the vegetative rosette over one winter paves the way for additional mutations that lead to perenniality. Again, these mutations would not be per se adaptive, because both winter annuals and perennials are fit in colder environments. However, other contingencies may transpire that result in extinction of the winter annual form, leaving warm-adapted annuals and cold-adapted perennials. Yet, at instantaneous points in this history, there never was adaptive temperature "selection." To the contrary, the mutations resulting in obligate vernalization were not selected and were maladaptive, because they restricted these mutants to the colder portion of the ancestral range. But in the colder environment, the mutations were effectively neutral.

The preceding demonstrates a mechanism that could lead to evolution of perennials from annuals. It also demonstrates that the developmental temperature sensitivity that gave rise to the perennial was a constitutive plastic response of the annual ancestor. Indeed, this resulted in mass reallocation below ground, but this was incidental to the response. Light was adequate for photosynthesis, but temperature was inadequate to trigger reproductive growth. Where else could the plant direct growth except toward the caudex? It was not a "strategy" intended to effect life history evolution, nor the cause.

The preceding is useful for interpreting phylogenetic evidence [5] indicating that the rosettiformcaudiciform perennial life history of Calyptridium umbellatum and C. monospermum is derived within the genus. These sister-taxa are nested within an otherwise annual clade with successive annual outgroups. The conclusion is supported by both molecular and morphological data. In particular, the perennial species pertain to $C$. sect.

31 Spraguea, a clade of three species, the other species being annual [1]. These species share at least three 32 morphological synapomorphies relative to other Calyptridinae: inflorescence bracts singular, leaves markedly less 33 succulent, and leaf veins not sinuous or laminar [5, 60, 61]. 
Hershkovitz

Montiaceae Ecological Evolution

Thus, the evolution of $C$. sect. Spraguea clade provides important evidence for interpreting Montiaceae life history evolution. It documents in a local (as opposed to geographically disjunct) context the origin, from an annual, of a long-lived rosettiform-caudiciform perennial species. This is contrary to the polarity derived in [3]. Moreover, as with C. guadalupensis, it demonstrates the rapidity with which this marked life history transformation can occur, rendering moot the relation between evolution and "branch length."

Finally, less obvious, $C$. sect. Spraguea clade documents not only rapid evolution of a long-lived rosettiform-caudiciform perennial, but possibly a fair portion of the reversion back towards an annual life history. The annual species of this complex is C. pulchellum, which is similar in size and form to other annual Calyptridium species: a small, ephemeral rosette with numerous spreading leafy inflorescence branches bearing small axillary cymes. The perennial species have a prominent and persistent rosette. They differ in that, compared to C. umbellatum, C. monospermum has traits characteristic of short-lived perennials and annuals. While its habit is unlike that of $C$. pulchellum, it has a rather small, slender, unbranched caudex , a prominent leaf rosette, and numerous axillary scapes per rosette, each bearing copious flowers. Calyptridium umbellatum has a thick and often branched caudex, each with a single terminal inflorescence [64]. But single-seeded fruits are unique to $C$. monospermum, which indicates that this species is derived.

Notably, C. monospermum accords with the evolutionary tendency derived in [3], even though Calyptridium as a whole does not. In particular, it appears to be an "annual-in-waiting" with a warmer temperature niche than $C$. umbellatum, with which it is known to hybridize and introgress [53]. And compared to C. umbellatum, C. monospermum allocates biomass preferentially to sexual reproduction rather than perennation. If the plant dies after one flowering, operationally it is an annual. All that is needed to be a true therophyte is programmed senescence after flowering. However, perenniality clearly is derived in Calyptridium, and the most diminutive species, C. pygmaeum, is an alpine annual.

The analysis above underscores the difference between narrative and PhCA approaches. Friedman [40] reviewed evidence for theories of life history evolution based on macroevolutionary and macroecological evidence. They recommended testing these theories not at the macroevolutionary (i.e., PhCA), but rather the microevolutionary scale, specifically in species rudimentarily polymorphic for life history. In this case, evidence from an annual Calyptridium species plastic for life history traits helps explain evidence for evolution of perennial species. The corresponding PhCA [3], in contrast, predicated to explain evolutionary causal mechanisms, but it deliberately restricted analysis to evidence remaining millions of years after the causal mechanism operated. Towards this objective, it sanitized species polymorphism at numerous levels, including its definition of parameter states, arbitrary polymorphism coding, and using statistical means as effectively discrete and fixed states of continuously variable and unfixed polymorphisms. This accomplished, it proposed as truth that manifested in frequent outcomes and disappeared observations of infrequent ones. 
Hershkovitz

Montiaceae Ecological Evolution

To be fair, however, within the PhCA paradigm, such machinations are not vices but virtues. They root in the ideals of statistical epistemology, in turn a mathematical form of essentialism. In this mindset, what is frequent is interpreted as the ideal evolutionary objective. It is as though the unpredicted infrequent events took a wrong turn in evolution and have been ostracized. As with all of its pseudoscientific numerological soulmates, it seems PhCA dismisses failure to retroactively predict not as the failure of its epistemology, but the failure of organisms to behave as they should.

\section{Montioideae: Rumicastrum, Calandrinia, and Hectorelleae}

These three taxa appear to be successive outgroups of Montieae [3, 4]. Hancock et al. [4] reconstructed the ancestral phenotype of Rumicastrum as annual, with perennials evolving 2-3X in the genus. This is contrary to the polarity derived in [3]. They did not reconstruct temperature niche evolution, but Australia lacks the extreme cold environments associated with Montiaceae perenniality in the Americas. But perenniality evolved at least twice in Rumicastrum [4]. There is no indication from the distribution of the perennial species that temperature was a factor in their evolution, as recognized in [3]. However, such indication might emerge from paleoclimate analysis.

Calandrinia includes both perennial and annual clades. The perennials all are alpine species, and the annuals occur at lower elevations, hence have warmer temperature niches. There are no exceptions, so Calandrinia is completely consistent with the tendency derived in [3]. However, the genus documents only a single life history transition, and the polarity is not certain. The ancestral node reconstructs as perennial in [3], but, again, this possibly is a reconstructive artifact. Molecular evidence does not corroborate the origin of the annual form from a perennial form, as proposed in [6].

All of the perennial species except the rhizomatous C. compacta are caudiciform, indicating that this is the ancestral form among the perennials. Some of the caudiciform species (e.g., C. acaulis) have the idealized [3] below-ground caudex and above-ground ephemeral leaves/inflorescence, while others (e.g., C. caespitosa) are cushion plants, with the apical portion of the caudex above-ground, highly branched, and suffruticose. The ancestral form is not established. However, the cushion forms have a more southerly distribution, ca. 32S-50S, whereas the acaulescent forms are distributed from about $40 \mathrm{~S}$ northwards to southern Mexico. This may be significant, because the southerly range was cold/cold since the Oligocene, whereas the alpine habitat in the northerly range developed towards the late Miocene to Pliocene [34, 35]. Interestingly, Hectorelleae also are cushion plants (see below).

Hershkovitz [5] proposed an evolutionary and phylogeographic scenario that does implicate ancestral perennial life history in Calandrinia. The clade comprising Calandrinia is sister to a clade comprising Hectorelleae, and Montieae, both of which are ancestrally cool/cold-adapted perennials. The perennial species of 
Hershkovitz

Montiaceae Ecological Evolution

1 Calandrinia are alpine plants distributed from Central America to Tierra del Fuego. The annual species are

2 distributed in environments rather cooler than annuals of the outgroups, Cistantheae and Rumicastrum. Thus, the

3 ancestor of "cool" clade of Montioideae may have been a tap-rooted caudiciform perennial situated towards

4 southern Patagonia during the Oligocene or Miocene. annuals in Calandrinia and Montiinae that is consistent with the conclusions in [3]. But caution always is advisable. As demonstrated above, multiple Montiaceae taxa demonstrates that the transition between diminutive annual and large perennial can involve little time and divergence. In fact, several such transitions appear in poorly diverged/resolved clades of extant species at the termini of relatively long branches. What went on during the 10+ million years prior to the diversification of the extant species cannot be ascertained. The evolution of alpine perennial Oriastrum provides a case in point [49]. As parsimonious as the life history evolution scenario elaborated above might appear, the distribution of life histories among the extant cladelets might well be luck of

\section{Montioideae-Montieae: Lewisia and Lewisiopsis}

These tap-rooted caudiciform taxa (with one exception) form a clade in [3] but are the successive outgroups of Montiinae in [4]. In [1, 5], I proposed that Lewisiopsis is a "living fossil" of an ancient hybridization between the precursors of Lewisia and Montiinae. This, however, does not affect the discussion here. Because Lewisiopsis and all Lewisia species are perennial, a perennial ancestor reasonably is inferred. The absence of annual species in this group is "statistically" peculiar, given its pedigree and geographic distribution. This taxon effectively is ignored in two-parameter analysis of the evolutionary relation between life history and ecology. Yet growth forms and climate niches are diverse among Lewisia species, hence provide useful information that must not be ignored.

Lewisia/Lewisiopsis species occupy temperature niches generally cooler that those of most annual Montiaceae, and many species are alpine dwellers. In the preceding section, I attributed this, with caveats, to their cool Patagonian ancestry. But, just as alpine species occur among more commonly warm-adapted annual Montiaceae, largely cold-adapted Lewisia includes relatively warm-adapted perennial species. One of them coexists in habitats overwhelmingly dominated by annuals (see below).

As mentioned previously, Lewisia sect. Cotyledon includes caudiciform "evergreen” perennials, i.e., their leaves persist year-to-year. They include the largest and largest-flowered species in the genus, and the leaves of most species are coriaceous rather than succulent. Their above-ground growth is much greater than in the ephemeral-leaved species. Most taxa are restricted to relatively cool, moist environments of the extreme northwest US, but these habitats are generally lower in elevation and decidedly warmer in winter than those of most alpine 
Hershkovitz

Montiaceae Ecological Evolution

Lewisia species. This suggests that, as in Cistanthe guadalupensis, shift to a warmer temperature niche can associate with different, even contrary, shifts in growth form traits. In the case of L. sect. Cotyledon, the aboveground organs are perennial, whereas in colder-adapted Lewisia spp., they are ephemeral ("annual").

The ecologically most remarkable species of Lewisia is the acaulescent ephemeral-leaved L. rediviva, the bitterroot, which can form large, long caudices. The species includes two varieties, L. rediviva var. minor, which is an alpine denizen, and L. rediviva var. rediviva, which spans elevations from subalpine to $60 \mathrm{~m}$. The latter has one of the broadest distributions of any North American perennial Montiaceae, extending from the northern and central Cascade and Rocky Mountains to southwestern California [41, 42, 63]. In California, at elevations below $500 \mathrm{~m}$, it occupies exposed rocky outgroups that can become are griddle-hot and bone-dry in summer.

The ecological restriction of bitterroot seems to be not so much temperature, aridity, or elevation as availability of a suitably rocky substrate. And this requirement itself might be indirect. In an edaphically mosaic site near Oroville, California, L. rediviva occurs in a habitat dominated by about 200 species of annuals of diverse lineages [64]. But it only occurs in tight crevices of flat rock outcrops. Presumably the long sinewy root permits access to deep moisture. It is not clear why the plants do not occur in the less rocky adjacent soil dominated by forbs and grasses. But this is beside the point, which is that evidently cold-adapted acaulescent caudiciform perennials indeed can evolve into warm, arid habitats dominated by annuals.

Although Lewisia includes no annuals, one species, L. triphylla, is diminutive and presumably shortlived. Superficially, above ground, it resembles Montia linearis. The species is tuberous, probably anatomically similar to tuberous Claytonia species. It occurs in montane to alpine habitats in seasonally wet microenvironments in gravelly substrate. It also counters the trend in [3] in that it demonstrates evolution towards ephemerality without evolving into a warmer or more arid temperature niche.

\section{Montioideae-Montieae-Montiinae}

Reconstructive methods are somewhat conflicting regarding the life history evolution among Montiinae. The threshold method, as applied in [3] inclines towards a perennial ancestor of Montiinae and both of its genera. This is in agreement with cladistic interpretation of morphology [6, 8]. But perenniality in Montia is rhizomatous/stoloniferous, and the ancestral form of perenniality in Claytonia seems to be most likely either rhizomatous or tuberous rather than tap-rooted caudiciform (see below). The latter result is unexpected.

All but three species of Montia are either stoloniferous/rhizomatous perennials or at least form adventitious roots at the nodes. Two stoloniferous/rhizomatous species of $M$. sect. Montia also form bulbils at the nodes. In one of these, $M$. parvifolia, the node-derived offspring superficially resemble acaulescent caudiciform plants. The strictly annual species are $M$. (sect. Montia) diffusa and the sister species M. linearis and $M$. dichotoma of $M$. sect. Montiastrum. Parsimony suggests that the annuals are derived. 
Hershkovitz

Montiaceae Ecological Evolution

There appears to be a precedent for rhizomatous perennials evolving from caudiciform in Calandrinia, a Montieae outgroup. The subaquatic rhizomatous species Calandrinia (sect. Acaules) compacta appears to have been derived from the morphology of $C$. caespitosa, a mat-forming cushion plant that differs from other perennial calandrinias in having a highly branched caudex. I cannot confirm whether or not its epicotyledonous caudex branches form adventitious roots. Calandrinia compacta and $C$. caespitosa occur alongside each other, with the latter species growing on the drier banks of bogs supporting the former. They occasionally hybridize [46]. The other Montieae outgroup, Hectorelleae, also is mat-forming. Whether Montiinae or all of Montieae originated as caudiciform-derived rhizomatous perennials is a matter of speculation. But indeed stolonifery and rhizomes among Montiinae species are associated with cool/cold boggy habitats.

Tap-rooted caudiciformy in Claytonia is derived at least once and possibly twice from epicotyledonous tuberous forms [27, 65]. One rhizomatous perennial species, $C$. washingtoniana, may have evolved from an annual, but might be a hybrid with rhizomatous $C$. sibirica [65]. There is no evidence demonstrating the origin of any annual from a caudiciform perennial, unless the root of Claytonia is thusly reconstructed. The evidence suggests that ancestral Claytonia was either tuberous or rhizomatous. Data from the sister group, Montia, corroborate better a rhizomatous ancestor. Again, this raises the question of whether the ancestor of Montiinae may have been a rhizomatous perennial derived from a caudiciform. But it must not be ignored, either that some Montiaceae perennials and biennials evidently also evolved from annuals. Presumably, an annual also could give rise to a stoloniferous/rhizomatous perennial.

Otherwise, there is evidence among Montiinae for the evolution from annual towards perenniality. OE [3] noted the occasional presence of small tubers in two (divergent) annual species of Claytonia (see also below). They characterized these as "vestigial," but they could as well be "rudimentary." Furthermore, Miller \& Chambers [66] remarked that the rosette of Claytonia rubra var. rubra may overwinter, analogous to that reported for Calyptridium parryi var. martirense [54]. Similarly, it possibly represents incipient evolution of caudiciform perenniality. This supports phylogenetic and empirical evidence from other Montiaceae demonstrating that perenniality can evolve rapidly in an annual species, which casts doubt on prevalent notions of Montiaceae life history evolution (see above).

The evidence introduces at least some question of the notion of ancestral caudiciformy in Montiinae. This perhaps is unexpected, given the prevalence of tap-rooted caudiciformy among Lewisia/Lewisiopsis. If the Montiinae ancestor was caudiciform, the trait was lost multiple times and rederived at least once. Regardless of the ancestral state, the (one or two) annual-perennial transitions in Claytonia involve rhizomatous, and not caudiciform or (caudex-derived) tuberous, perennials. The ramets are or may behave as, effectively, opportunistic annuals. Thus, rhizomatous and, more so, bulbilliferous forms are not equivalent ecologically to long-lived caudiciform perennials. In fact, as noted above, ecological equivalence even of developmentally-related 
Hershkovitz

Montiaceae Ecological Evolution

caudiciform and tuberous perennials is questionable. O'Quinn [39] hypothesized that the latter facilitated evolution of tuberous Claytonia species into warmer temperature niches.

Ecologically, Claytonia contributes significantly to the evolution of the overall Montiaceae temperature niche breadth. Several species extend or are endemic to subarctic zones. Phylogenetically, Claytonia has extended to these latitudes at least five times [65]. Outside of Claytonia, only three Montia species and Lewisia pygmaea s. 1. extend to this zone (none endemic; $[63,65]$ ). At the other extreme, species of $C$. sect. Claytonia extend to warm, arid SW US and humid arid SE US [27, 67]. Two species of Claytonia extend into Mexico [67].

Notably, Claytonia diversification takes Montiaceae ecological evolution almost full circle. On one side, Claytonia culminates the phylogenetic trend of Montiaceae towards cold Arctic environments. But on the other side, the range of species of $C$. sect Claytonia and the annual $C$. perfoliata subsp. mexicana in SW US and Mexico bring Claytonia back into the range of Cistantheae and Phemeranthus, the outgroup of cis-cordilleran Montiaceae. In fact, these taxa take Montiaceae to more humid S/SE US, bringing Montiaceae into the range of other Portulacineae. Although the local niche of Claytonia species in these regions might not be shared with the other warm-adapted lineages, the geographic overlap creates the potential for greater niche convergence possible.

Also notably, Montiinae includes six of the eight Montiaceae species that have spread naturally or anthropogenically beyond their native western North American range [1, 5]. These include the sub-cosmopolitan Montia fontana, M. chamissoi, M. linearis, M. parvifolia, Claytonia perfoliata, and C. sibirica.

Returning to the analysis and conclusions in [3], the data show general agreement, but with caveats. All species of the colder high altitude/latitude environments are perennial. Annual species of both Claytonia and Montia indeed occur at lower elevations than perennials, hence presumably in warmer environments. However, it appears that the few long-lived tap-rooted caudiciform perennials evolved from shorter-lived tuberous ones. At the same time, the tuberous forms, while characteristic of cooler environments, did evolve also into warmer environments. In addition to presenting evidence for secondary derivation of tap-rooted caudiciformy, Montiinae also present evidence of incipient perenniality in annuals. Both phenomena are documented in other Montiaceae.

\section{Summary}

Applying a stochastic phylogenerative method, OE [3] found that perennial Montiaceae species in various genera of Montiaceae all evolved from annuals. Applying reconstructive methods, they inferred the reverse, which they accepted as "more realistic." The perennial to annual scenario is the conventional interpretation $[6,8]$. A case-by-case analysis demonstrates that the annual-to-perennial transition occurred several times. Just as importantly, current phylogenetic evidence does not demonstrate unequivocally that the acaulescent rosettiformcaudiciform perennial growth form is ancestral in any genus with both perennial and annual species, or even in perennial taxa polymorphic for growth form. To the contrary, the phylogenetic evidence indicates that this growth 
Hershkovitz

Montiaceae Ecological Evolution

form evolved from an annual form in three genera (Calyptridium, Rumicastrum, 3-4X in Cistanthe sect. Rosulatae). Except for Montiopsis subg. Montiopsis, all species of clades presumably ancestrally caudiciform (Phemeranthus, Montiopsis subg. Dianthoideae, Calandrinia sect. Acaules, Lewisia/Lewisiopsis, and Claytonia sect. Claytonia) are entirely perennial. Verification of the ancestral life history of Montiopsis awaits additional phylogenetic resolution.

Why, then, did the perennial life history repeatedly reconstruct at ancestral nodes of Montiaceae genera with both annual and perennial species? One reason is phylogenetic irresolution in minor clades with both life histories. Notably, however, resolution of some minor clades may not be possible [3,5]. Another is the assumption of homology among developmentally different manifestations of perenniality. Distinguishing annual, rhizomatous, acaulescent, and caulescent caudiciform growth forms as unordered states likely would have affected reconstruction of the Montiinae ancestral node with consequent affects on deeper nodes.

Without verifying per se the theoretical/technical bases of the phylogenerative analysis, its conclusion is more "realistic" than it appears. First, some of the diagnosed cases of annual-to-perennial transition probably are correct. Second, it optimally fitted the Portulacineae phylogeny with a "two-rate" stochastic model of life history evolution, one fast, one slow. This yields four qualitative rates, i.e., fast versus slow rates of annual to perennial and vice versa, depending on the phylogenetically incidental life history. The assumption of slower evolution in perennials [10] yields an expectation of less frequent perennial-annual and more frequent annual-perennial transitions. This seems to be corroborated. Moreover, the annual-perennial transition appears to have occurred over short evolutionary time, and some annuals manifest incipient perenniality.

In multiple instances among Montiaceae, modern life history/form transition coincides with modern temperature niche transition. Annual species commonly distribute in temperature environments warmer than their most closely related perennial relatives. This observation emerges independent of evolutionary polarity. The phylogenetic correlation between life history and temperature niche gradient led [3] to conclude that phylogenetic life history lability itself allowed Montiaceae to rapidly diversify into and among temperature environments.

Montiaceae indeed manifest a broad temperature niche distinct from that of other Portulacineae, and indeed the incidence (as distinct from "rate") of phylogenetic temperature niche diversification is likewise higher. But the narrative analysis demonstrates that the putative correlation between life history and temperature niche is more complex than it appears. Perennials and annuals as collective categories each span much of the total temperature niche range observed among Montiaceae. One tap-rooted caudiciform perennial species alone (Lewisia rediviva) spans a broad swath of this breadth. Another, Montiopsis umbellata, ranges from warmest low elevation to coldest alpine elevations at its latitude. With no change in life history - or growth form -- species of Claytonia sect Claytonia spread across much of the North American continent, coast-to-coast and from central Mexico to the Arctic Circle. Perennial Phemerantheae diversified among cool temperate and warm tropical habitats without changing life history. In at least two phylogenetic instances, annuals gave rise to perennials that 
Hershkovitz

Montiaceae Ecological Evolution

occupy a warmer temperature niche. As many as five times, annuals evolved into the alpine zone. Other annuals range from warm temperate to cold temperate environments. These one-off observations demonstrate that nothing intrinsic to Montiaceae life histories restricts their temperature niche.

As [3] noted, interpretation of the relation between life history and ecological evolution among Montiaceae is contingent upon the inferred directionality of life history evolution. Derivation of annuals indeed implicates novel evolution into warmer habitats. But the reverse implicates retention among annuals of the warmer temperature niche shared widely among Portulacineae outgroups. In this case, the derived perennials would have expanded the niche into colder habitats. Or, at least, this scenario would have created the biogeographic pattern in which considerable perennial species diversity occurs in colder (including alpine) habitats.

Narrative analysis supports a notion, contra [3], that a life history transition better explains the diversification of Montiaceae into colder habitats than the reverse. Even though Portulacineae and Phemerantheae are perennial, the first and most pleisiomorphic lineages of cis-cordilleran Montiaceae, Cistanthe and Calyptridinae , are mainly warm- and arid-adapted annuals. The life history transition from the ancestor was "neutral" with respect to temperature niche, although many Phemerantheae evidently are more cold-tolerant than annual Cistantheae. In the latter, multiple cases of an annual-perennial and associated temperature niche shift are well-supported.

Meanwhile, Montiaceae present evidence of annual-perennial evolution via genetic assimilation. This involves flowering-time cold temperature-sensitivity, leading to fixation of a vernalization requirement in winter annuals, leading to the elaboration of perennials. This is evident in cases in Calyptridium and Claytonia. But obviously this specific mechanism cannot explain the de novo evolution of warm-adapted perennials, such as Cistanthe guadalupensis. Likewise, modern evidence does not implicate frigidity in the evolution of perennial Rumicastrum species, although frigidity might have played a role at the time these taxa originated.

Narrative analysis suggests that a key transition facilitating temperature niche diversification of modern Montiaceae may have been the evolution of a cool-adapted perennial that situated in southern Patagonia during the Oligocene [5]. From this ancestor emerged the cool-adapted taxa Calandrinia, Hectorelleae, and Montieae, Phylogenetic evidence suggest that this ancestor may have been mat-forming and possibly rhizomatous rather than strictly tap-rooted. This may have been a key factor in subsequent diversification.

Montieae and, especially Montiinae, then succeeded conquering a grand tract of the cool temperate northern hemisphere (since there was little area in Patagonia to conquer). And from there, Montieae reentered their ancestral warm, arid habitat, especially in California. This evolution involved both annuals (Claytonia and Montia) and tap-rooted caudiciform perennials (Lewisia). And they spread to southern and eastern North America in the form of tuberous plants. Montieae also returned to the southern hemisphere apparently $2-3 \mathrm{X}$ in the form of node-rooting Montia species. 
Hershkovitz

Montiaceae Ecological Evolution

Whatever characteristics might seem to seem to coincide with Montiaceae diversification, one or another exception will demonstrate that that characteristic is neither necessary nor sufficient to cause diversification. For example, as noted in [3], Montiaceae diversified in regions of temperate America whose ecological gradients are steep seasonally, diurnally, and spatially, and have been exceptionally dynamic through recent geological history. Did this, by itself or in conjunction with morphological/developmental characteristics, "cause" Montiaceae diversification? Why, then, are Lenzia and Lewisiopsis monotypic?

The narrative analysis also permits appreciation that transitions in both life history and temperature niche occur across (in several cases unmeasurably) short evolutionary divergences. In several cases, temperature niche and life history/form appear polymorphic within species. The significance is two-fold. Firstly, it indicates that evolution of these traits does not distribute along "branch lengths," as many PhCA methods presume. Evidently, the trait states in more divergent taxa offer little or no power to predict diversity of these traits among closely related taxa or even within species. This is ironic, because the diversity of these traits at low divergences among Montiaceae evidently precisely vetted their suitability for PhCA (see also below). Secondly, the narrative analysis predicts a pattern of both broader and narrower temperature niches within and among closely related species, with no change of life history or growth form.

The preceding is corroborated narratively for both annual and perennial species. The widespread Lewisia rediviva provides an excellent example, because 13/17 species in this genus have locally restricted ranges [41, 42, 63]. Likewise, a few Montiinae species have especially broad distributions while closely related species are narrowly endemic. And some Montiaceae species, especially Montiinae, have become adventive more globally (see above), likewise extending their original temperature niches. Also, subspecific taxa, presumably recently derived, generally have differing climate niches. Examples include subspecific taxa of the relatively rare species Lewisia cantelovii, L. columbiana, and L. cotyledon [41, 42, 63], of Claytonia spp. [27], and of Calyptridium parryi $[54,55]$. Probably no less than a dozen cosmopolitan angiosperms weeds have temperature range phenotypes inclusive of nearly all Montiaceae combined. The significance of this observation with respect to PhCA will reemerge in the final discussion.

These narrative observations impugn the applicability of statistics in evolutionary analysis. In particular, [3] calculated much higher rates of temperature niche and life history diversification of Montiaceae relative to their outgroups. From a descriptive perspective, this is accurate at this scale. But unlike conventional statistical estimates, this is not an estimate of an underlying "true" rate whose precision increases with increased observations/trials. Within Montiaceae, some taxa have experienced markedly less cladogenetic and ecological diversification than their Montiaceae sister taxa. These include the monotypic and ecologically restricted genera Lenzia and Lewisiopsis, and the ditypic Hectorelleae. Sister groups of these taxa have at least 23, 23, and 85 distinct (sub-)specific taxa, respectively [1] and correspondingly broader niche breadths and growth form 
Hershkovitz

Montiaceae Ecological Evolution

diversity. And reiterating from above, cladistic diversification evidently is not prerequisite for ecological diversification.

The narrative analysis (above) suggests that life history evolution among Montiaceae has been idiosyncratic, i.e., lineage and circumstance specific. Thus, in a lineage- and circumstance-specific ("one-off”) manner, life history changed or it did not. Lineages diversified cladistically and/or ecologically or they did not. Alternative historical contingencies would have generated alternative histories. The calculated overall "rate" of life history evolution is a statistical artifact of historical contingency. The narrative analysis also indicates that, when life history transforms, it can do so rapidly, and that, in several, if not most, cases, the transformations among Montiaceae have been in both directions.

Montiaceae demonstrate that, while the conclusions of PhCA are not necessarily "wrong," they can be misleading and are, in any case, deeply impoverished. This owes to the necessary optimization for statistical precision, which necessitates artificial conversion of complex parameters into coins and minimizing sampling of variability. In the case of Montiaceae, this manifests in conversion of multiple growth forms and their intermediates into two somewhat arbitrarily coded life histories. And it manifests in the reducing of temperature niche data to a mean of a continental- or global-scale distributions.

PhCA [3] concluded that temperature niche transitions generally accompany perennial-annual transitions, with annuals occupying warmer and perennials occupying cooler niches. Narrative analysis corroborates this generally, but also demonstrates exceptions. And infraspecific variability ignored in $[3,12]$ demonstrates that life history transition is not necessary to incur such shifts. Does the PhCA statistical significance provide anything other than a precise but not especially accurate estimate of relative frequency? Indeed, the PhCA might beg an explanation as to why one transition is more common than another, but the frequency data evident from narrative analysis seems to serve this function equally well.

The narrative analysis reveals data nuances not accommodated in the PhCA of [3]. This includes recognition of the variability of temperature niche in widespread species, obviously monomorphic in life history. By making only interspecific comparisons based on somewhat arbitrarily assigned temperature niches, $\mathrm{PhCA}$ might have overestimated interspecific temperature niche shifts and hence the overall phylogenetic correlation of temperature niche shifts with life history shifts.

The narrative analysis also recognized the difference between plant microenvironment an ambient air temperature. This notion was not incorporated into the present interpretation, because no data are available. But it was not considered at all in [3]. It seems reasonable to presume that both summer/winter temperature minima/maxima would differ between exposed rocky outcrops and boggy or otherwise temperature-buffered plant microenvironments. Then again, it would seem reasonable to presume that accomplished and well-funded plant ecophysiologists would know this. Thus, it is possible that the present discussion completely missed the point of the analysis of the evolutionary relation between plant traits and ambient temperature data in $[3,11,12]$, and 
Hershkovitz

Montiaceae Ecological Evolution

hopefully these authors will clarify this point in the future. In any case while PhCA found a significant correlation between life history and temperature niche shifts, it bases on incomplete and probably inaccurate temperature data. Is mathematically sophisticated and computationally intensive but inaccurate precision better than accurate but unsophisticated narrative analysis?

Ultimately, the objective of PhCA is to explain evolution causally. The PhCA in [3] explained the correlated increased rate of Montiaceae cladistic and ecological diversification as a consequence of lability of above/below-ground biomass allocation implicit in life history lability. No biomass allocation data were provided, but the hypothesis is testable. The narrative analysis indicated that transitions in above/below-ground biomass allocation associated with transitions between particular growth forms often are not coincident with transitions in life history. The PhCA did not notice this, because Montiaceae growth form evolution lacked the foresight to optimize itself for its eventual statistical analysis.

\section{Moisture niche evolution among Montiaceae}

In addition to temperature niche, OE [3] and also Smith et al. [12] analyzed evolution of "precipitation niche" in relation to life history and plant succulence across the Portulacineae phylogeny. In contrast to temperature niche, [3] found no difference in phylogenetic correlation of precipitation niche and life history diversification between Montiaceae and the rest of Portulacineae. They found some phylogenetic correlation between precipitation niche and evolution of succulence, which will be analyzed further below.

OE [3] did not equate precipitation niche explicitly with moisture (or hydrological) niche, though it seems implicit. The implication is more pronounced in [12]. There seems little other reason to analyze precipitation niche except as a moisture niche proxy. But $[3,12]$ they did not note that precipitation niche is a very crude and often inaccurate predictor of moisture niche. For example, even aquatic plants live in desert aquatic environments with very low precipitation.

The approach in $[3,12]$ and similar PhCAs has several limitations, of which I list three below and others later:

(i) Precipitation does not necessarily predict the hydrological environment of the plants. In the high cordillera near Santiago, Chile, succulent species of Cistanthe occur on dry northern exposure slopes while perhaps 100 meters away Calandrinia compacta and Montia fontana grow in bogs. Even if life history and/or succulence are tightly coupled with moisture availability, which they are not, these observations demonstrate that moisture availability varies at a microecological scale somewhat independent of the local precipitation scale. Again, it seems that successful ecological researchers would know this. So again, it is possible that the present discussion completely missed the point of analyzing the relation of plant trait 
Hershkovitz

Montiaceae Ecological Evolution

evolution with precipitation data in [3, 12], and hopefully these authors will clarify this point in the future. In any case, the method of $[3,12]$ seems to assume that moisture niche and precipitation. are tightly coupled.

(ii) Modern precipitation levels likely are not indicative of past precipitation levels and fluctuations. The method of [3] effectively assumed two possibilities: (a) modern taxa dispersed into their modern precipitation niches, evolved phenotypes accordingly, and maintained their precipitation/phenotype status ever since; or (ii) phenotypic evolution occurred in situ in response to precipitation evolution, and taxa maintained this phenotype/precipitation relationship ever since. A rudimentary understanding of the multiple temporal dynamics in play renders this scenario naïve. These dynamics are evident in [59], which overlaid historical diversification of Chilean Oxalis with historical environmental development. This should render clear that the (supposed) precision of modern climate locality data emphasized in $[3,12]$ and similar PhCAs hardly compensates for the lack of precise distribution/climate data during the course of diversification. To the contrary, this approach seems more likely than not to misinterpret evolutionary history, precisely because of the mega-annual-scale data gap between explanandum and explanans.

(iii) Even ignoring both of the above, [3] did not note that annual precipitation means are misleading in areas affected by El Niño, which encompass much of Montiaceae diversity. Especially in central and northern Chile (and to a lesser degree, California), precipitation is bimodally distributed. Relatively few rainy years are separated by often decade-long droughts. This affects all plants, but perennials more than annuals. Annuals in arid Chile tend to germinate only in infrequent wet years.

However, the motive for the modern wave of PhCA analysis of precision precipitation locality data might owe less to its plausible utility than its amenability to statistical manipulation and ready availability in a database. $\mathrm{PhCA}$ is a part of a paradigm rooted more in statistics than in biology. This facilitates a predilection to analyze statistically any readily available data, even if they are biologically meaningless.

Ironically, while [3] found no significant departure of Montiaceae from Portulacineae moisture niche evolution, narrative analysis shows that Montiaceae exhibit a range of moisture niche markedly and remarkably different from other Portulacineae. To be fair, [3] were concerned primarily with differences in rates of evolution, especially with respect to life history evolution, but their results like include analytical artifacts, especially from using precipitation as a moisture proxy and from using means as proxies for phenotypes in widespread species. Narrative analysis indicates that these sophomoric errors indeed obscured what is likely significantly higher moisture niche diversification among Montiaceae relative to Portulacineae.

Montiaceae exhibit a phylogenetically successive trend towards a more humid environment and eventually back again towards a more arid environment. Phemerantheae, the sister group of cis-cordilleran Montiaceae, also are mainly arid-adapted. As noted above, [33] described Phemeranthus species has having 
Hershkovitz

Montiaceae Ecological Evolution

"legendary" drought resistance. But other species distribute in areas of moderate precipitation, and one species, $P$. humilis, is subaquatic [68].

Cistantheae, especially Cistanthe and Calyptridinae, are, like their Portulacineae outgroups, xerophytic. Narratively, in my travels, I have found Philippiamra celosioides to be the last plant encountered when approaching "the driest place on earth" in the Atacama Desert in southern Region II in Chile. This holds whether approaching from the northwest or southeast. Retreating from here, other Philippiamra species also are among the few plants encountered. Species of Cistanthe occur somewhat further away. Thus, $P$. celosioides is a candidate for the most drought-resistant of all herbaceous angiosperms.

Even in environments with higher precipitation, Cistantheae prefer drier sites compared to more derived Montiaceae. For example, the central Chilean/Argentinean alpine species Cistanthe frigida and C. picta are restricted to dry slopes, whereas nearby Calandrinia species occur in bogs and pools. Several Montiopsis species are present, and these also prefer drier slopes and well-drained flats.

At the other extreme, Montiaceae, especially Montiinae, include many true hydrophytes, a characteristic otherwise absent among Portulacineae. Montia fontana sometimes grows as a floating aquatic, not rooted in soil [69]. Other species of Montia, Claytonia, Lewisia, and Calandrinia grow in bogs. Many species of Montieae indeed inhabit higher precipitation zones in the NW US and SW Canada, which comprise the wetter end of the Portulacineae precipitation spectrum. But the hydrophytic species of these and other Montiaceae occupy wet sites even in zones considered xerophytic by precipitation measurements. At the same time, Montiinae genera with hydrophytes also include closely related more arid-adapted taxa. Arid-adapted species (or subtaxa thereof) include Claytonia exigua, C. perfoliata, C. rosea and other tuberous Claytonia species [27, 67], Lewisia brachycalyx, L. maguirei, and L. rediviva [42]. Consequently, some Montiinae have returned to their ecological ancestral roots, becoming sympatric with species of Phemeranthus and Cistantheae.

There does appear to be a partial correlation between life history and moisture niche among Montiaceae and seed plants in general. In particular, while both annuals and perennials occupy xeric and mesic environments, (sub-)aquatic plants usually are perennials. But the relation is not absolute, evidenced by Montia fontana, which, if not an ideal annual, at least appears to be ephemeral. Otherwise, wetland- and bog-inhabiting Montiaceae, like hydrophytic angiosperms generally (including mangrove species), are perennial. However, annual species may be associated with vernal pools, emerging and reproducing along their receding margins [64]. Calandrinia menziesii and Claytonia perfoliata subsp. perfoliata are naturally a vernal pool margin species that have spread as adventives into irrigated cultivated areas, as well as areas of moderately high precipitation [1].

OE [3] also attempted to explain the evolution of succulence among Montiaceae in terms of precipitation. The same authors had published previously an excellent review of plant succulence phenomena, noting the various anatomical and physiological forms and functions of succulence [70]. In that work, they appeared to recognize that succulence was determined by factors in addition to aridity. In particular, they illustrated what they 
Hershkovitz

Montiaceae Ecological Evolution

believed was marked infraspecific plasticity in succulence in plants of Cistanthe grandiflora growing meters apart. They attributed the difference not to precipitation or even moisture per se, but exposure to salt not in the drier, but in the wetter and more maritime microenvironment. As it turns out, the difference was not plasticity. The more succulent plant illustrated is C. laxiflora. Confusion of the latter with C. grandiflora owes to historical misinterpretation, which I also had propagated [1]. But indeed the illustrations demonstrate differences in succulence of closely related plants growing nearer and further from seawater.

Notwithstanding the phenomenologically pluralistic vision of succulence offered in [70], [3] proposed that phylogenetic transitions towards succulence among Montiaceae should be negatively correlated with mean annual precipitation, but positively correlated with precipitational seasonality (i.e., wet and dry seasons). The premise of the latter was that succulence allowed Montiaceae to store episodically available water. Their analysis confirmed the predicted correlation between succulence and annual precipitation, but not precipitation seasonality.

Factors overlooked in [3] (even if acknowledged in [70]) mitigating an expected phylogenetic relation between (modern) succulence and (modern) precipitation those are listed above, e.g., demonstrating why how succulent and nonsucculent plants might coexist in different hydric microenvironments within the same precipitation niche. The list of overlooked factors continues below:

(iv) As OE noted in [70], the premise that succulence must be associated with aridity is unwarranted. They illustrated an example from Montiaceae. Succulent species too numerous to list of Portulacineae (especially Cactaceae), other Caryophyllales, and other angiosperms thrive in conditions of moderate to high precipitation. Some succulent species, including Phemeranthus humilis and Lewisia triphylla are relatively hydrophytic. In halophytes like Salicornia (Chenopodiaceae), succulence is associated with excess, albeit saline, moisture.

(v) Part of the arid region concentration of succulents may owe as much or more to their slow growth/reproduction, hence competitive disadvantage in wetter climates dominated by more rapidly growing plants. Overcoming this handicap, phanerophytic succulents like Opuntia spp. (Cactaceae) hold their own in regions of moderate to moderately high precipitation. Thus, the relation between succulence and aridity may be more an indirect effect of growth rate than adaptive advantage per se of succulence.

(vi) Some succulent taxa, including some succulent Montiaceae and other Portulacineae, are restricted to fog desert formations. Precipitation data capture inadequately or, in the absence of weather stations, completely fog moisture data and its physiological effects. Fog deserts and fog-influenced beach microenvironments in Chile support several Montiaceae and other Portulacineae (esp. Cactaceae) taxa, while nearby adjacent arid areas are devoid of vegetation. Yet, their precipitation data may be identical or very similar. Obviously, precipitation data alone can distort the apparent phylogenetic relation between 
Hershkovitz

Montiaceae Ecological Evolution

phenotype and environment, in this case misleadingly suggesting that succulent plants occupy habitats much more arid than they actually are.

(vii) Even to the degree that succulence among Montiaceae adapts to aridity, other Montiaceae exhibit alternative adaptations, some of which were recognized in [70]. In above-ground growth, these include dense foliar trichomes (Montiopsis spp.) and epidermal specializations such as thick cuticles and sunken stomata (Cistanthe spp., Lewisia spp.) and broad, scarious leaf margins (Lenzia). Such specializations adapt plants to arid regions by mechanisms other than water conservation [71]. In addition, below-ground structures in Montiaceae, other Portulacineae, and many lineages of plants, adapt plants to aridity, as O'Quinn [39] proposed for tubers in Claytonia. Consequently, plants may transition phylogenetically between succulence and other xerophytic adaptations within the same aridity environment. Analogous to the reduction of multiple growth forms to two life history traits, classifying plants only as succulent or nonsucculent may mislead evolutionary analysis of the ecological evolution.

Before summarizing, one particular example of succulence vs. aridity evolution in Chile stands out. While Tropaeolum is not considered to have succulent stems/leaves, the term has been used in some taxonomic description of stems/leaves of some species [72-75], and I use the term here to demarcate notable differences within the genus. Tropaeolum subg. Chilensis includes ca. 25 basically cis-cordilleran species distributed from the Atacama Desert to Tierra del Fuego [74, 75]. The "arid region clade" comprises three (taxonomically problematic) entities that are patently nonsucculent. Their lengthy vine stems, small leaves, and flowers are as thin, delicate, and "dry" as can be. The outgroups include similar but slightly meatier-stemmed vines of moister lowlands extending south to the Chilean cool rainforest, and a clade of central/southern Patagonian montane/alpine taxa that have more markedly succulent stems and leaves/flowers rather meatier than other species. The more diverse Tropaeolum subg. Tropaeolum is distributed from semiarid eastern South America northeastwards through the altiplano region and along the cordillera to Central America. One species, T. majus, has become an invasive species in adequately humid frost-free habitats throughout the world. Well-irrigated plants of this species can have markedly succulent stems and at least somewhat fleshy leaves. Thus, Tropaeolum appears to demonstrate the opposite of the trend presumed in [3]: evolution of succulence in inverse relation with precipitation!

I do not predicate to explain here the evolution of succulence among Montiaceae. Rather, I show how expedient statistical oversimplification, second-hand data, and inadequate field knowledge can mislead PhCA, whether the results appear to corroborate dogma (e.g., correlation of succulence and total precipitation) or not (e.g., succulence and precipitation seasonality not correlated). The results yield, at best, a crude systematic description, not an evolutionary explanation. Most succulent Montiaceae indeed are also xerophytes, so a correlation with precipitation total and seasonality would not be unexpected. However, Montiaceae manifest 
Hershkovitz

Montiaceae Ecological Evolution

adaptations to aridity other than succulence, and these intersperse with succulence phylogenetically. By lumping mesophytes with non-succulent xerophytes, [3] could not have detected phylogenetic transitions between different xerophytic adaptations, e.g., between succulence and hairiness among Cistantheae. However, diagnosis of all adaptations to aridity would have complicated the PhCA technically and added parametric categories. This, in turn, would have reduced the statistical significance with which correlates of succulence might have been detected.

In summary, PhCA of precipitation data failed to capture the complexity Montiaceae moisture niche evolution. It is not clear that the latter was the objective of the PhCA. But the parallel comparative analysis of temperature and precipitation renders apparent no other objective. Precipitation breadth does not discriminate between Montiaceae and Portulacineae as whole [3], but moisture niche departs markedly. Moisture niche among Montiaceae is much broader than among the remaining Portulacineae combined, and practically spans that of all angiosperms combined. Indeed, at lower phylogenetic levels, Montiaceae do appear to manifest more rapid moisture niche diversification than other Portulacineae. Montiaceae manifest a strong phylogenetic trend from xerophytic towards hydrophytic, but some derived taxa reversed towards xerophytic. PhCA of precipitation versus plant succulence seems logical. But xerophytic species do not always manifest succulence, and succulence does not always associate with xerophyty. Thus, even if PhCA had detected phylogenetic correlations, these would reflect incidental frequency and not causality.

Thus, narrative analysis demonstrates both a marked phylogenetic shift and enhanced evolutionary lability in moisture niche among Montiaceae compared to other Portulacineae. PhCA [3] found no shift and no enhanced lability owing to analysis of precipitation data rather than the ecology of the taxa. And, as with the temperature analysis, this may reflect partially the conversion of infraspecific precipitation breadth of widespread species to means,. This demonstrates the pathology of computational approaches to ecological/evolutionary analysis dating back to the implementation of such methods throughout the past half century ago (e.g., [76: 64, 77, cf. 78]. In particular, computational evolutionary analysis requires no familiarity with the organisms per se. It only requires organism-tagged data. Now, thanks to Bayes-turbocharged supercomputers fueled by bottomless global data pumps, computational approaches can generate meaningless, misleading, and absurd conclusions faster and, unlike narrative analysis, with greater statistical certainty than ever.

\section{Polyploidy and ecological diversification among Montiaceae}

Using a similar approach as [3] and exploiting the same climate databases, McIntyre [11] and Smith et al. [12] studied the relation between polyploidy (whole genome duplication) and climate niche diversification among Montiaceae. McIntyre [11] studied the relationship in three subspecific taxa of the Claytonia perfoliata species complex. Smith et al. [12] studied the relationship across the order Caryophyllales, including evidence from 
Hershkovitz

Montiaceae Ecological Evolution

Montiaceae, especially Claytonia. The premise, articulated in numerous theoretical essays and reviews (e.g., [12]) is that doubling of all genes in a genome enables genomic functional flexibility and specialization of duplicated genes, leading to increased phylogenetic diversification. The reviewed essays also note, however, potential deleterious effects of polyploidy (see below).

Notably, [12] did not distinguish between autopolyploidy and allopolyploidy. The premise of polyploidyinduced diversification rests on the supposed consequence of genome duplication. It is best tested in autopolyploids and not allopolyploids. This is because the former essentially are otherwise identical to diploid progenitors. The latter, which result from hybridization, are novel biological entities not equivalent to either diploid parent. Allopolyploidy instantaneously creates new genotypes without divergence and specialization of duplicated genes (although this may occur as well). Smith et al. [12] analyzed ancient polyploidization events, in which an auto- versus allopolyploid origin cannot be discerned, as well as recent ones. The difference is important, however. Hybridization itself, with or without polyploidy, is widely supposed to facilitate phylogenetic shifts into new habitats [79-83]. Because of subsequent genotypic segregation, it also can increase the rate of phylogenetic diversification. Furthermore, allopolyploidy is considered to be far more common than autopolyploidy [80].

The present work rejects the premise of [12] on both technical and epistemological grounds (see below). But even without these objections, the failure to distinguish between allopolyploidy and autopolyploidy invalidates a test of a hypothesis of phylogenetic consequences of, specifically, polyploidy, without considering the effects of hybridization. Given the vast literature on both hybridization and polyploidy, it is puzzling how the significance of this distinction could have been overlooked among all authors and reviewers. It further exemplifies the pathology (see above and below) spawned by the trend towards scientific analytical automation, which privileges method over reason and artificial intelligence over human intelligence.

A more notable point, in the present context, is that the analyses of $[3,11,12]$, attempt to interpret the same evolutionary phenomenon in the same plants in terms of different traits, i.e., life history and polyploidy. OE [3] did not mention polyploidy nor note that life history is constant in C.perfoliata, while its cytotypes differ in climate niche [84]. Polyploidy occurs in many Claytonia species [85], both annual (2-10x) and perennial (2-12x). But $[11,12]$ likewise did not consider in detail other possible explanations for observed ecological diversification; [12] mentioned one life history shift coincident with polyploidy among Nyctaginaceae, but did not mention the frequent change of life history among Montiaceae.

Another notable point is that polyploidy represents the most extreme form of genetic duplication mutation (whole genome duplication in whole progeny), whereas all forms yield both potentially positive and deleterious effects on phenotype and diversification. At the other extreme are single nucleotide duplications; intermediate are replication- and transposon-induced duplications of loci and whole or partial chromosomes. And all forms can be found throughout the organism or just in individual cells (e.g., developmentally regulated transposition 
Hershkovitz

Montiaceae Ecological Evolution

1 duplications and genetically programmed endopolyploidy). Thus, the category "polyploid" is arbitrary, analogous

2 to but less well justified than the category "annual life history" (see above). The contrary state, "not polyploid,"

3 includes all manner of genetic duplication except whole genome duplication in whole organisms. Thus, PhCA of

4 the relation of polyploidy to diversification only partially partitions the underlying characteristic supposed to

5 correlate with the latter.

Evidence from [11,12] is inconclusive with respect to the theoretical prediction that polyploidy causes ecological diversification, though the conclusions of [12] invalidate themselves in any case for failing to discriminate between genome doubling and hybridization effects. But even ignoring this, inconclusiveness is not surprising. In their review of evidence, Soltis et al. [82, 83] defend this premise, but the studies they cite sometimes do and sometimes do not support it. More broadly, the eukaryotic phylogenetic spectrum is not wanting of examples of highly polyploid organisms that have "gone nowhere" evolutionarily, not morphologically, not ecologically. These examples include some of the most extreme of polyploids, 100-1000x (e.g., the fern genus Ophioglossum).

In the context of Montiaceae, it is notable that diploid chromosome numbers of Hectorella (Hectorelleae; 2 spp.) and the monotypic Lewisiopsis are approximately 96 [41, 86], suggesting at least octaploidy. Both taxa are reasonably close relatives of (though considerably older than) Claytonia [3], a genus showcased as an example of polyploid-induced diversification [11, 12]. Yet Hectorelleae and Lewisiopsis manifestly are under-diversified and, moreover, they are highly restricted geographically and ecologically [41, 42, 86]. On the basis of uniformitarianism, these data falsify the premise of polyploidy-induced diversification. In fact, Hershkovitz [1,5] suggested that Lewisiopsis is a "living fossil" of ancient allopolyploid origin, and that allopolyploidy actually sequestered subsequent phenotypic evolution.

Contrapositively, there is no shortage of examples of taxa that have diversified morphologically and ecologically without the benefit of numerous independent and successive polyploidizations. I cite the example of the Hawaiian silversword alliance (Asteraceae; Madiinae), regarded as one of the most spectacular examples of morphological/ecological adaptive radiations. The group evidently evolved from a single allotetraploid and probably unspectacular "damn yellow composite" perennial herbaceous founder, but without subsequent polyploidization [87]. However, tetraploids (axiomatically) are pervasive throughout angiosperms, and I believe it is safe to say that generally tetraploidy has not yielded such dramatic morphological/ecological diversification. Diversification of the silversword alliance must owe to other factors.

Thus, narrative analysis demonstrates that, as for any other character, polyploidization is neither necessary nor sufficient to potentiate evolutionary diversification. Polyploidization (like all mutations) axiomatically causes organisms to differ, but (like all mutations) it does not impose any particular qualitative/quantitative morphological/ecological distinction, nor does it preordain subsequent polyploid survival, proliferation, or diversification. 
Hershkovitz

Montiaceae Ecological Evolution

The question remains, however, as to why phylogenetic mapping studies render an impression of the evolutionary potentiation of polyploidy. Smith et al. [12] found both correlated and uncorrelated instances of polyploidization and ecological diversification. They suggested that the latter partially might owe to the broad scale of their analysis, hence inadequate precision and data at finer scales. But they also invoked an earlier proposal that ecological diversification axiomatically must transpire after the diversification stimulus, in this case polyploidization. Hence, departures from coordination between polyploidization and diversification may represent evolutionary lag times. However, Smith et al. [12] appeared to recognize the inherent speciousness of such a proposal, as it cannot be falsified on the basis of the historical sequence.

It must be emphasized (whether or not a novel observation) that both polyploidization and diversification are historically co-linear geometric growth functions. Indeed, both trace to the same cause: the imperfect process of DNA replication during cell reproduction. Strictly speaking, neither process is reversible, though other processes counteract them (i.e., extinction and polyploid diploidization, respectively). Over time, lineages derived from polyploids inevitably will increase, and eventually all lineages are likely to have polyploidy in their pedigree. Meanwhile, obviously all lineages are products of diversification events. Varying degrees of temporal coincidence is to be expected of historically co-linear processes. Thus, alignment of polyploidization/diversification events might not be positive evidence for causal relation, but failure to align is negative evidence.

Indeed, polyploidization represent a major mutation, so it is not to be unexpected that phylogenetic shifts are temporally associated with polyploidy. But this correlation is somewhat artificial, because the effect is observed only when polyploid lineages indeed diversify. There may be a determination bias in play in interpretation of polyploidy consequences, because macroevolutionary researchers tend to focus on more rather than less diversified taxa, hence less diversified polyploid lineages will be ignored. Also, it must be emphasized that genome duplication, like macromutation generally, usually is maladaptive. Both auto- and allopolyploid mutations result in inviability or sterility. Otherwise, it is unlikely that polyploids are instantly fitter in their incident environment than their adapted diploid progenitors. Arguments for the phylogenetic superiority of polyploids, therefore, are somewhat specious, basing on the relatively few successes and oblivious to the true magnitude of failure. It is analogous to touting the effectiveness of a medical treatment based on the cure rate of the small proportion of patients it did not actually kill.

As a final note, a relevant twist on the relation between life history evolution, climate niche evolution, and polyploidy emerges in [45]. These authors concluded that the perennial life history in the genus Castilleja (Orobanchaceae) evolved from the annual. This evolution appeared to be associated with polyploidy. The authors believed that this observation corroborated earlier suggestions that deleterious effects of polyploidy favored vegetative rather than sexual reproduction, hence prolongation of the vegetative growth phase, hence perenniality. One consequence is an increase in generation time. Contemporaneously, Smith \& Beaulieu [10] found 
Hershkovitz

Montiaceae Ecological Evolution

correlations between herbaceous/woody and faster/slower rates of both molecular and climate niche evolution, and also found that herbaceous lineages evolved into a broader climate niche space than woody lineages. They attributed these observations to the faster generation time of herbaceous lineages. But Smith et al. [12] subsequently argued that polyploidy promotes climate niche evolution -- even though polyploidy is associated with increased generation time, which they had argued results in slower climate niche evolution. Go figure.

\section{CONCLUDING REMARKS: STOCHASTICITY/DETERMINACY VERSUS IDIOSYNCRATICITY OF EVOLUTION}

In the decades following Felsenstein's [15] seminal paper, PhCA has developed into the principal paradigm of macroevolutionary theory (e.g., $[13,14]$ ). This is consequent not only to its theoretical logic, but especially to the increasing availability of fine-scale molecular phylogenies across the tree of life, increasing availability of biodiversity data in public databases, increasing development of computational theory and methods, and increasing computational power. PhCA now is applied routinely to study biological mechanisms in paradigms ranging from ecology to cell biology.

The motivation of PhCA generally appears to be discovery of Darwinian determinants (“drivers") underlying biological diversity and diversification. But the conclusions are coupled tautologically to theoretical and methodological assumptions and biases, such as those described earlier in this work. Also, as demonstrated in this work, different theoretically justified PhCA methods yield different, even diametrically opposed, evolutionary conclusions. Exceptions to statistical correlations are not reported or not explained or explained ad hoc.

In this section, I recapitulate and expand discussion of how myriad assumptions and biases of PhCA generate a misleading, if not erroneous, interpretation of the nature of evolution. For example, one unappreciated bias that PhCA introduces into evolutionary interpretation is consequent to the requirements of statistical analysis generally. Requisite for PhCA include, first of all, adequate phylogenetic "replicates." It also requires sufficient phylogenetic polymorphism of multiple homologous traits. PhCA cannot be applied to traits that are invariant or hardly variable across the phylogeny. It cannot either be applied to traits that are hypervariable (polymorphic within terminals). Nor can it be applied to traits with too many trait states of uncertain biological and/or evolutionary relations, or traits whose states cannot be unambiguously defined/diagnosed. In cannot be applied in such cases, but it is. In such cases, as I described above, PhCA tends to resolve polymorphism and define/diagnose trait states artificially and arbitrarily for statistical expedience, yielding misleading conclusions.

Consequently, the PhCA paradigm inherently overestimates the degree of correlation between trait evolution, environmental variability, and phylogenetic diversification. This is partially because the paradigm emphasizes studies of taxon/trait data sets which potentially conform to its premises. Phylogenetic trait correlations emerge from a subset of these. PhCA excludes analysis of taxon/trait data where it is inapplicable or 
Hershkovitz

Montiaceae Ecological Evolution

poorly resolvable. Thus, the theoretical/empirical importance of particular traits as essential drivers of evolution may reflect a combination of statistical cherry-picking and data distortion. It is to be expected that evolution does not optimize itself for statistical analysis. And there does not appear to be any organismal trait or environmental influence both necessary and sufficient to determine the course of evolution. Yet, no trait or influence can be considered evolutionarily irrelevant. The instantaneous viability of organisms is a function of the instantaneous integration of their traits with their environment. Instantaneous phenotypic function thus decouples from phenotypic evolutionary trajectory. Metaphorically, organisms can evolve and chew gum at the same time.

To illustrate the preceding, [3] selected Montiaceae for PhCA specifically because of their phylogenetic variability in the analyzed traits, including climate niche traits. The premise was to study the relation of climate niche and phylogenetic diversification. I suggested above that at least a dozen cosmopolitan angiosperm "weeds" representing diverse lineages each occur throughout most of the combined climate range of Montiaceae (and then some). Diversification of nearly 300 species over 40 million years was not necessary for these species to achieve the same climate niche breadth. Yet, because such species are not phylogenetically diversified, their climate niche breadth is not apportioned discretely among separable lineages. Thus, evolutionary analysis of their niche breadth is not amenable to PhCA. Their phylogenetic rate of climate niche diversification is undefined. Their variability is out of the PhCA paradigmatic radar range. At best, their "mean" climate niche trait values will appear in PhCA (as in $[3,12)$.

Likewise, angiosperms offer examples of taxa phylogenetically diversified but more or less monomorphic for climate niche. Consequently, PhCA-thinking renders more conspicuous patterns such as that of Montiaceae, in which trait and taxonomic variability appear to apportion. Yet, taxa "invisible" to PhCA indicate that phylogenetic diversification is neither necessary nor sufficient to cause climate niche diversification, and that climate niche diversification is neither necessary nor sufficient to cause phylogenetic diversification.

$\mathrm{PhCA}$ also inherently focuses on taxa and trait combinations only when the trait polymorphism appears positively correlated phylogenetically. However, other taxa lack those phylogenetic trait correlations (one polymorphic but not the other) or even inverse correlations (polymorphism increases phylogenetically for one trait while the other decreases). For example, reproductive morphological polymorphism generally is associated with adaptive radiation. Among Montiaceae, variability in reproductive morphology is maximal among the ca. 23 species and subspecific taxa of Lewisia/Lewisiopsis. The species vary considerably in inflorescence morphology (form, branching, size, flower number, floral formulae), floral size, sepal morphology, petal color, and ovule number [41, 42]. Yet, as noted above, geographic ranges of most Lewisia species are especially small, and the plants are not especially common. No adaptive value is obvious. Possibly PhCA practitioners focus on evolutionary "successes" rather than "failures," hence might overlook this apparent increase in polymorphism and decrease in ecological range and frequency. It does not fit the narrative of reproductive morphological adaptive radiation. 
Hershkovitz

Montiaceae Ecological Evolution

A contrary example is the Lewisia/Lewisiopsis sister-group, Montiinae. Montiinae life history/form and

possibly also karyotypic diversity potentially accords with the narrative of the relation between these and temperature niche breadth/diversity. Moreover, many of the species have ranges an order of magnitude or more greater than most Lewisia species. One is cosmopolitan and five others spread anthropogenically. They are ecologically "successful." But reproductive morphology of Montiinae compared to other Montiaceae is notably monotonous. Thus, paradoxically, in this case, PhCA logic would suggest inverse correlation, hence negative causal relations, between reproductive morphological diversity and temperature niche breadth/diversification. But evolutionary analysts might be biased against perceiving and testing such inverse correlations. Constant characters do not call their attention to statistical analysis.

Thus, the PhCA paradigm is prone to generate analytical epiphenomena. It conditions a search image for trait distributions that conform to its premises. Analysis of such biased trait distributions confirms its premises. But a key observation is that narrative analysis finds exceptions to the rules that PhCA tends to impose. I explain this observation in the context of what I term the principle of evolutionary idiosyncraticity (PEI). As I elaborate below, this principle bases on evolution as a stochastically-perturbed chaos-like process driven by organismal determinism as conceived in the theory of non-Darwinian evolutionary ND [23]. PhCA, however, roots in the statistical Darwinian evolutionary paradigm founded in the theory of natural selection (NS). Conventional PhCA and PEI are epistemologically incompatible, although PhCA can be applied heuristically under PEI.

However, to appreciate the utility of PhCA under PEI, it is necessary first to appreciate the roots of statistical PhCA and essentially the entire contemporary systematics paradigm from within the statistical Darwinian paradigm. Then it is necessary to reiterate that the statistical Darwinian paradigm is pseudoscientific. And so goes the entire contemporary systematics paradigm.

\section{The roots of PhCA and contemporary systematics in the statistical Darwinian paradigm}

The thesis presented here emphasizes cultural rather than philosophical/epistemological origins of the contemporary PhCA and systematics paradigms. The latter are deliberately glossed over, because it is reasonably evident that ideological radiation has as much or more to do with political/economic history as philosophy/epistemology. In practice, the latter are applied dogmatically according to political/economic expedience. And this thesis should reasonably self-evident to contemporary plant systematists whose careers date back to the early 1980s or earlier, when phylogenetic taxonomy was just emergent, when word processors were pencils and typewriters, and when the entire DNA and protein sequence database fit onto a few pages of text.

Classical "evolutionary" taxonomists of this age professed to be Darwinists, but this denomination was misleading. Evolutionary conceptualization was not phylogenetic, but Aristotelian, i.e., according to Scala Natural [88]. Organisms were classified according to whether they were intrinsically "primitive" or "advanced," and taxa 
were referred to as being "lower" or "higher." The only difference was that evolutionary taxonomists professed that taxa were related by genealogical descent, even if their evolutionary arguments were utterly incompatible with this premise. Likewise, many classical taxonomists professed to pertain to the Darwinian Modern Synthesis paradigm. The latter incorporated both physical and statistical evolutionary genetics and the new paradigm of "biological systematics," which was supposed to bridge genetics with macroevolution.

But the more quantitative/experimental biosystematics focused on species-level taxonomy and had little practical impact on macrotaxonomy. In fact, biosystematic data itself could be and often was incorporated seamlessly into classical taxonomy. Thus, the alliance of classical taxonomy with Darwinian evolutionary biology largely was mutualism/commensalism. Taxonomy provided evolutionary biology with taxon identifications and names. Evolutionary biology often provided taxonomists with phenotypic and geographic data. But otherwise, the paradigms were fairly segregated. Even within the field of systematics, the more statistical biosystematics and classical taxonomy segregated. For example, the official journal of the American Society of plant Taxonomy, Systematic Botany, spun off in 1978 from its predecessor, Brittonia. The former was more quantitative/experimental and the latter was more classical taxonomic/floristic. More revealing, the former was ISI indexed, the latter was not.

The paradigm of phylogenetic taxonomy, or cladistics, emerged in the 1960s-1970s from taxonomy and not from the Modern Synthesis. This is reasonably evident especially in the criticisms of cladistics by Modern Synthesis patriarch and patently biosystematical Ernst Mayr, and the broader eschewing of cladistics by most of the contemporary plant taxonomic and biosystematics community up until at least the mid-1980s [89-91]. This was ironic, because Darwin clearly conceived of evolutionary diversification as a cladistic process. Thus, the then-hegemonic evolutionary taxonomy school was betrayed as non-Darwinian. This hardly helped the esteem of taxonomy in the biological discipline more broadly.

But the establishment of phylogenetic systematics owed mainly to factors other than epistemological enlightenment. One was simply generational turnover. As simple as was cladistic logic, it was incomprehensible to many conceptually classically-schooled taxonomists. But unindoctrinated students learned cladistic language and logic easily. A second factor was more political. Unlike narrative classical taxonomy, cladistics explicitly appealed to scientific method. It was quantitative and computational and predicated to discriminate between true and false taxonomies. A third factor was technological/economic. Prior to the mid 1980s, computational analysis in taxonomy was carried out on mainframe computers. By the end of the 1980s, fully graphic user-interfaced cladistics software permitted easy point-and-click cladistic analysis and even publication-quality illustration preparation. This advance, as much as or more than epistemology, sealed the future of phylogenetic systematics.

The preceding account underscores an important principle. Science advances as a popular front, influenced only indirectly by theory and epistemology, but mainly by socioeconomic and political factors far removed from these. Indeed, scientific evolution itself is idiosyncratic. As a case in point, early in the 
Hershkovitz

Montiaceae Ecological Evolution

1 epidemiology of cladistic taxonomy, the mere implementation of a computer validated taxonomic work as

2 scientific and credible, no matter how incompetent and nonsensical they were. Perhaps more so if the author was

3 especially politically influential, e.g., a program director of a national science funding agency.

Part of the credibility afforded emergent research results owes to the "naked emperor effect." Especially in systematics, the broader research community is not sufficiently educated to evaluate newly emergent research theory/methods, especially applied to taxa which with they are not familiar. But scientists do not like to betray their ignorance, so they will tend to lavish praise on such works and accept whatever conclusions they offer as highly credible if not true. They will not heed the evaluation of a competent critic because, if they do not understand the new research, neither do they understand the criticism. Quite possibly, pseudoscience has a selective paradigmatic advantage in systematic and evolutionary biology.

This was demonstrated by a 1980s - vintage computational phenotypic cladistic analysis of Caryophyllales [92], its theoretical and taxonomic execution thoroughly invalidated in [77], and its phylogenetic conclusions, if anything, negatively correlated with current evidence [2]. Yet, it was cited authoritatively and uncritically in subsequent Caryophyllales research. Remarkably, even 30 years later, the familial circumscriptions applied in [92] were considered referential because they emerged in the "first" cladistic analysis of Caryophyllales. But, as pointed out in [77], the familial circumscriptions applied in [92] were not derived or validated cladistically. They were noncladistic circumscriptions used in classical references. Evidently distracted by the cladistic and computational aesthetic of [92], none of the 22 Caryophyllales systematics/phylogenetics experts authoring [2] noticed this discrepancy. In the institution of biodiversity (pseudo)science, scientific style often trumps scientific substance.

In the meantime, another school of phylogenetic systematics emerged from within the statistical Darwinian paradigm. This based on ML, which, like PhCA, was introduced to phylogenetics by Felsenstein [93]. The cladistic paradigm emergent from taxonomy was quantitative and computational, but generally it eschewed statistics, because phylogeny was considered a unique event, not a sample from a population of phylogenies. The taxonomic school advocated the criterion of parsimony. In the age of primarily morphological data, the statistical phylogenetics approach did not viralize. The exception that proved the rule was widespread use of the bootstrap in parsimony analysis. Again, this test did not emerge from the taxonomic school, but the statistical school by, once again, Felsenstein [94].

But ML required considerable statistical training to understand. It was implemented in software, but not in point-and-click graphic software, and it was extremely slow. And it was derided by the taxonomic cladistics school. More importantly, ML analysis requires prior estimation of the probability of trait transitions, which in turn requires large amounts of polymorphic data and coin-like traits. Most morphological cladistic data emphasized traits unique to small numbers of taxa, and many traits were complex and not easily modeled statistically. 
Hershkovitz

Montiaceae Ecological Evolution

All of this changed with advances in DNA sequence based phylogenetics in the 1990s. Maximum likelihood models of DNA sequence evolution had been developed within the statistical paradigm beginning 20 years earlier. By the mid-1990s, a particular ML implementation had been incorporated into point-and-click software and otherwise identical command-line versions were portable to multiple operating systems, including those of high speed computers and computer servers. Development of Bayesian estimation afforded lightening speed to likelihood analysis with the added advantage of resolving phylogeny with apparently very high certainty.

Researcher preferences shifted in favor of statistical phylogenetics. Not only was statistical phylogenetics now fast, easy, and apparently surefire, its users became kindred with the broader statistical evolutionary biology paradigm. The Modern Synthesis now was fully consummated and consolidated. Meanwhile epistemological arguments of the parsimony school were forgotten, if they ever were understood in the first place. Researchers adopted MP when it was the most expedient, and abandoned it when something else assumed this distinction. Researchers are much more pragmatic than philosophical. By the time ML was popularized, the parsimony school had essentially exiled itself out of mainstream academic evolutionary biology.

This brief history helps explain why PhCA is essentially statistical evolutionary population genetics applied to "populations" of taxa, observing trait polymorphisms and testing hypothesis of Darwinian determinism (i.e., NS), but correcting for genetic relatedness of the "individuals" manifest in their phylogeny. But exactly as in the statistical evolutionary population genetics paradigm from which it is derived, evolution is measured as a nonrandom shift in the mean value of a dependent phenotypic trait value, and its cause is interpreted in terms of covariation with one or another independent variable.

It is important to appreciate that issues raised in the present work and in $[16,25]$ are not especially relevant in the statistical PhCA paradigm, just as they are not relevant in statistical evolutionary population genetics. The statistical paradigm recognizes only statistical arguments, not narrative ones. The statistical PhCA paradigm is not concerned with: (i) extreme phenotypic values in observed in analyzed taxa, because taxa, as individuals, can be represented by their means; (ii) phylogenetically contrary observations, because these are axiomatic variance having no evolutionary significance; (iii) inadequate or even biased sampling, as long as the bias is not systematic and/or systematic bias (e.g., phylogenetic relatedness) is corrected; and (iv) expedient parametric dimensional consolidation (e.g., growth forms to life histories), because the objective is to discriminate phenomena statistically rather than ontologically. Statistical epistemology is intended to provide a means to test reasoned hypotheses in terms of probability distributions. It does not, nor predicate to, adjudicate the a priori reasonability of hypotheses. Reasonability is adjudicated tautologically by statistical support.

\section{Natural selection and statistics: dumb and dumber}


Hershkovitz

Montiaceae Ecological Evolution

The subtitle of this section is a deliberate double entendre. Literally and scientifically, it is true. NS was and has remained an explanation of naturally improbable biological engineering and diversity by means of "unintelligent design." Hence, the NS is literally "dumb design." Statistics, meanwhile, posits that the default explanation of phenomena is random chance, i.e., it has no cause at all. A random outcome presumably is even dumber than one selected, however unintelligently.

However, the more cynical meaning is the intended one. Firstly, mainstream evolutionary analyses generally presume that the adaptive NS narrative is true. The failure of empirical observation to conform with its expectation generally does not engender epistemological challenge. Secondly, over the past three decades, the macroevolutionary biology paradigm embodied in PhCA has become increasingly technology-dependent, exploiting ever larger data sets ever more automatically generated/mined and analyzed. These technological trends reflect a deliberate shift towards artificial intelligence approaches to evolutionary biological research. A consequence of epistemologically sterile artificial intelligence is that it appears to obviate the need, perhaps even the desirability, for natural human intelligence [cf. 78].

In any case, this work takes the view that Darwin's theory of adaptive NS, at least in the simplistic form popularly conceived in texts and empirical research, has been discredited theoretically/empirically so thoroughly as to obviate any need for further rumination. Especially discredited are the conventional statistical Darwinian paradigm and, independently, the applicability of Darwinian adaptationist interpretations at the macroevolutionary level. Thus, popular applications of PhCA, as in [3, 12], synergize the most discredited aspects of evolutionary theory. However, the Darwinian paradigm remains predominant in empirical population-level and, with $\mathrm{PhCA}$, macroevolutionary biological research.

Criticisms of Darwinian theory are referenced below in minimally necessary detail. Justice cannot be done to the theme here. Those familiar with the debate need not review the evidence. Those unfamiliar would be wise to investigate. Several criticisms are summarized and referenced in [95: 4]. Additional salient criticisms include [22, 23, 96-100]. The listing is not exhaustive. Collectively, these cover the gamut, theoretical and empirical, from the demonstration of non-Darwinian evolutionary mechanisms to arguments that adaptive NS as envisioned by Darwin is rare to nonexistent and/or theoretically implausible given the vicissitudes of phenotypic and environmental complexity and dynamics. Other works criticize, specifically, naivety of statistical approaches to evolutionary analysis. Notably, some criticisms originated long ago within the Darwinian paradigm, some dating back to Darwin himself, e.g., whether NS was the exclusive evolutionary mechanism.

Sometimes, fundamental concepts, such as the meaning of selection, are debated within the NS paradigm. An example is Franks [101], addressing criticism of the value of the Price Equation in NS theory. This equation sometimes has been cited as effective proof of NS (e.g., [102]). Franks [101, and earlier works] has emphasized that the Price Equation is a trivial yet crucial and widely applied means of bookkeeping. It calculates demographic changes in one or more trait frequencies across a single population generation in a way that permits estimation of 
fitness of ancestral individuals according proportions of descendent individuals possess those traits. This corresponds to presumptive selection. Essentially, it detects and quantifies allometric or biased demographic change.

But the general equation applies to all forms of presumptive selection, including artificial and nonbiological selection, e.g. annual changes in the frequency of automobile models sold or continuous variables, such as the change in average fuel efficiency. The equation only infers whether "selection" has resulted, not whether it has actually occurred in the Darwinian sense (see below). To appreciate this, consider the automobile example. A change in the frequency of a particular model or in overall fuel economy might reflect customer demand (presumptive deliberate selection). But it could reflect myriad factors and not reflect presumptive selection at all, even though the net result is the same. Economics of industrial production is complicated. So is evolution. Gould [26] made equivalent points in criticizing the tautological equation of evolutionary change with proof of adaptive NS. The Price Equation may have been a brilliant innovation [101], but it does not explain its results. Functionally, it is just a "dumb" equation.

A underappreciated semantic issue emerges here, and its importance is discussed below. Because the Price Equation measures only allometric or biased demographic change, its denomination as a measure of selection is meaningful only in the context of presumed Darwinian NS. It is now widely appreciated that other evolutionary mechanisms can have the same consequence. In fact, Divine intervention can cause the selection effect. Price happened to have conceived of his solution to selection measurement in the context of evolutionary biology. But he recognized that his equation was applicable to all forms of demographic change and sought to develop a general theory of selection [101]. This effort was terminated by his psychological demise and untimely death. Given the evidence, I speculate that this, in turn, owed to his realization that "selection" obligates a "selectivity," as there cannot be such a thing as "nonselective" selection. This, in turn, would have revealed to him that Darwinian NS theory was a tautology having no scientific basis. It also raises the question of whether his death indeed was a suicide. Maybe he knew too much.

The simplistic summary conclusion here bases on evidence that NS is neither necessary, nor sufficient to explain evolutionary change. It also bases on the observation that the statistical NS paradigm, which purports to prove NS, appeals to statistical and not biological epistemology. Statistics summarize biological descriptions, but offer no biological explanations. In the case of NS, being statistically dependent, statistics obligate the incarnation of replicates, conceived as individuals in abstract populations that are considered analogous to physical biological populations. The latter itself is a concept as troublesome as the species concept.

In particular, empirical NS analysis necessitates populations of conspecific individuals that are independent and identically distributed and presumptively equivalent except for one or more polymorphisms of interest. This presumes, among other things, a stable hierarchical relation between tangible species and populations, as well as tangibility, functional integrity, and continuity of natural (as opposed to 
Hershkovitz

Montiaceae Ecological Evolution

artificial/experimental) populations. These assumptions are unrealistic. In the end, theories must be proven empirically. Evidence for NS derives not from empirical evidence, but NS theory-biased/constrained/contrived assumptions and observations.

Some of the reviewed criticisms NS-based PhCA (see below) are reflected in the narrative analysis of Montiaceae evolution here and in [5]. These include interpretation of causal origin of phenotypes in terms of their apparent function millions of years later and the interpretation of statistical covariance as explanatory rather than descriptive, a tendency in evolutionary biology that annoyed even Sewell Wright [103]. Also included is the conception of taxa as, after phylogenetic correction, equivalent independent and identically distributed individuals in populations whose polymorphisms can be proxied arbitrarily and/or by arithmetic means. I also suggested that observed phylogenetic correlations could have been annulled or reverted in other possible phylogenetic histories.

This refers to the dilemma of statistical analysis of a phylogeny, a singular historical event, conditioned only itself [17]. PhCA does not consider the spectrum of possible outcomes generated by hypothetically replaying the evolutionary tape, as suggested by [104] and demonstrated empirically by [105].

Ironically, the most definitive rejection of NS emerged in its own defense in a barrage of criticism of antiDarwinian evolutionary arguments [106-110]. Pigliucci [109] wrote:

“....contrary to popular belief, natural selection is not an optimizing process - [which explains] why [natural selection] makes mistakes and is inefficient, yielding whatever outcome is good enough for survival and reproduction."

This is a remarkable assertion because it describes not Darwinian, but effectively neutral evolutionary theories, including those that Darwinists historically derided (see below). Evidently, Darwin himself "misunderstood Darwin" [cf. 106], lest the subtitle of his Origins [111] would have been "...The preservation of 'good enough' races in the struggle for life." To render Darwin's position clearer, he subsequently favored the term "survival of the fittest (and not "survival of the adequate")" as better conveyance of his theory [112]. With NS (and axiomatic common descent), Darwin predicated to solve two outstanding questions of the origin of biodiversity: the Malthusian paradox of population stability and the remarkably complex adaptations of organisms to their lifestyle. The only alternatives at that time were Lamarckian inheritance of acquired adaptive traits or intelligent design.

\section{Natural selection as scientific truth: based on science or global politics/economics?}

Why did [109] propose a definition of NS that accommodates practically every evolutionary theory except Darwin's? And why did [106] assert that a non-Darwinian evolutionist misunderstood what Darwin could not 
Hershkovitz

Montiaceae Ecological Evolution

1 have made clearer? No scientific explanation seems plausible. A more plausible explanation has to do with

2 balance of global political power, and to some extent the immediate socioeconomic interests of evolutionists.

A scientific explanation of the "good enough" definition might refer to the difference between Darwin's explicit definition of fitness in terms of survival capacity and the modern definition based on reproductive success. But that is no help, because Darwin linked survival to phenotypic superiority in order to solve the Malthusian paradox. This necessarily links NS to reproductive success. Dead organisms have zero reproductive fitness. The same applies if fitness is defined in terms of reproductive success, because reproductive success cannot increase in all lineages, unless resources infinitely increase. That is why pyramid investment schemes do not work. In fact, reproductive success ranges between 0 and $\infty$, so asymptotically it indeed converges on the survival criterion. Moreover, there is no possible way to define selection nontrivially except in terms of optimization. If organisms differ in their heritable traits, what could selection be selecting if not those most optimal per the selective agent?

Actually, the explanation of Pigliucci's [109] assertion is simple. Pigliucci evidently understood that Darwinian theory is obsolete, if not wrong [e.g., 113]. Its defenders increasingly concede larger roles to nonDarwinian mechanisms [114], moving NS towards the margin but never off the page. But Pigliucci evidently understands the magnitude of the political dilemma consequent to recognizing that NS is false. Indeed, it has the potential of being apocalyptic.

For more than a century, Darwinists have debated ideologists promoting Biblical creationism. More precisely, have promoted Darwinian adaptive optimization NS as the scientific explanation for the origin and design of biological diversity, including humans. Other evolutionary mechanisms are recognized, but these are explicitly segregated from NS in evolutionary research and education literature and debate.

The debate is politically polarizing worldwide and of no small consequence. The alternative viewpoints are deliberately shaded with political overtones. Evolutionists associate creationism with right-wing extremism, which is illogical, since nothing in right-wing ideology implicates Biblical creationism or even deism. In fact, Nazi leaders, including Hitler, were anti-Biblical. Creationists link evolution with political Marxism, which is highly logical, since Marxism is atheist, which inherently negates creationism. But, political philosophy and the evolution question aside, aside, deism and atheism induce natural political animosities.

Not to be overlooked is the considerable degree of socioeconomic comfort/prestige afforded by statefunded scientific research. With institutional infrastructure included, exceptionally few researchers could finance their activities and lifestyle personally. Especially in abstract sciences such as evolutionary biology, adhesion to popularized, hence marketable, epistemologies is socioeconomically a more conservative strategy than venturing into more exotic theory.

Thus, power politics and economics, and not science, fuel the evolution debate and the defense of NS. Otherwise questions of origins and evolutionary mechanisms have little practical value in the course of human 
lives or history. Any moral posture can be accommodated by any theological/humanist/scientific philosophy. Racist/sexist/homophobic policies have been defended on Darwinist grounds, and creationists do not deny the principles of artificial selection or genetic organismal modification. Political policies are manufactured in their respective factories and marketed under whatever ideological brand sells best. Political strategy never has been inhibited from coopting popular religious doctrines.

Thus, in the bigger picture, the evolution/creation debate is known popularly as the science/religion debate. And indeed the evolutionist argument emphasizes Darwinism and statistical evidence for NS. The demise of Darwinist dogma leaves evolutionists in a compromised position. They would have to admit to creationists that their "science" was wrong all along. Then they would have to defend their belief in evolution with no empirical or even cohesive and comprehensible logical argument to demonstrate its mechanism.

And this underscores the fallacy that the debate pits religion against science. It is a debate between religions. Perhaps up to a billion adults worldwide would concede to the notion that humans are descendents of “apes." Perhaps no more than $0.001 \%$ of evolution adherents worldwide are sufficiently knowledgeable and experienced in evolutionary theory and empirical research to critically evaluate alternative evolutionary theories. Even within the biodiversity and general biology disciplines, I have known more than a few colleagues whose deist convictions are much stronger than their Darwinist. Thus, >>99\% of evolution adherents base their belief not on their own scientific experience and knowledge, but on a largely indoctrinated faith in the religion of humanistic scientism. I suspect that many more deists have experienced "miracles" than evolutionists have experienced "evolution."

At the same time, while the Darwinists' attitude towards creationists has been condescending, their attitude towards non-Darwinian evolutionists has been derisive. Non-Darwinists have been ridiculed and labeled as "outsiders" [102, 114]. Non-Darwinian evolutionary theory has been considered a social menace [102], perhaps even more so than creationism. But this animosity is not uncommon in science, even among the most qualified of experts. But most scientific controversies have little or no political consequence, whereas no issue in science is at once so politically sensitive and scientifically intractable as evolution. Unwittingly, the Darwinist intransigence has rendered vulnerable not only its own position, but possibly that of science in general, as explained below.

The demise of NS presents ominous possibilities. Especially thanks to PhCA, the statistical Darwinist paradigm now dominates the entire state-funded biodiversity research industry, both economically and politically. I emphasize that PhCA does not inherently obligate NS adhesion, as I demonstrate later. Rather, the paradigm inherited notions and methodology from the statistical Darwinist paradigm, and this canalizes its orientation operationally. The demise of NS, especially the statistical incarnation, raises the specter of (justifiable) betrayal of considerable biodiversity research as pseudoscience and consequent undermining of credibility of the scientific institution and the justification of its funding. 
Hershkovitz

Montiaceae Ecological Evolution

As it happens, because of its disciplinary hegemony, the statistical Darwinist paradigm has intersected with what has become the other currently most politically sensitive scientific debate: global climate change and its political and economic ramifications. As a consequence, considerable biodiversity research has come to be justified economically under the umbrella of climate change science and other conservation issues of practical importance. This, in turn, has fomented a degree of economic opportunism among researchers, as they struggle to retool/rebrand their research according to prevailing funding opportunities [115]. It is not uncommon to find in PhCA publications supposed consequences for climate change. PhCA by the most prominent of researchers published in the most prominent of journals have been enveloped in spurious climate change theses, especially blatant examples being [116, 117].

This scenario might seem far removed from PhCA of Montiaceae, but it is not. It explains why [3, 12] even exist. PhCA is a product of the Darwinian NS paradigm. NS is bundled into its epistemology and its methodology, as though no other evolutionary interpretation was possible. Consequently, it canalizes how evolutionary research is executed -- how evolutionary data are collected and analyzed. And it canalizes interpretation - adaptive or not adaptive. Few researchers criticize this approach, and their criticisms are ignored. But it is not because NS is true or even plausible. It is because of the cascade of political and economic ramifications that its abrupt rejection might engender.

Pigliucci [109] recognized the problem. Pure science appeals to pure reason. Institutional science appeals to institutional reason. Aligning the two is a challenge. Presumably with noble intentions, [109] resolved the problem by discretely swapping a non-Darwinian definition of NS for the widely accepted Darwinian one, and hoping nobody notices. Why not? Rebranding contrarian ideologies as "democracy" historically has been politically effective. This approach has apparent advantages at all levels and for all intents and purposes. It allows existing research to carry on under the Darwinian brand, whether or not it is Darwinian. A propos the creation/evolution debate, it avoids the revelation that "science" has been wrong all along and does not have yet the answer to what is right ("Watch this space?"). So the definition switch maintains scientific institutional credibility. And it repels outsider auditing of the scientific institution and the consequences of whatever this might reveal.

The approach also avoids discarding the baby with the bathwater. It allows pupation and metamorphosis of the evolutionary biology discipline, so that its mass can be digested and reconstructed in a new epistemological form and with new operational methodology. When the imago emerges, obviously it will be no longer the Darwinian NS larva. But hopefully it will be sufficiently spectacular that nobody will care or even remember. At that point, the term NS will become obsolete. In the meantime, the preferred strategy is to not destroy the larva.

I suggest a different approach. With all due respect for weaning evolutionary biology off NS, if anything threatens the credibility of science, it is not inaccuracy, but disingenuousness. In particular, creation science might be branded as pseudoscience, but ultimately it unapologetically founds in faith, which, by definition, is beyond 
Hershkovitz

Montiaceae Ecological Evolution

the scientific realm. NS pseudoscience, alternatively, bases on a scientifically falsified theory. Science based on or appealing to theory/evidence known, or even suspected, to be scientifically inadequate or false goes beyond pseudoscience. It creates the conditions whereby the principal obstruction to scientific advancement is none other than the nominal scientific institution itself.

The viewpoint taken here is that, while NS is dumb and its statistical verifications dumber, many researchers themselves are not so dumb. For those motivated more by the passion, if not obsessive-compulsion, to "know," than by their institutional socioeconomic standing, the demise of NS presents tremendous opportunity to discover, especially in the realm of PhCA. This is because evolutionary research at the mechanical level takes time to percolate to the level of systematics and macroevolutionary biology. A propos, it took more than 50 years for Modern Synthesis statistical principles and methods to become broadly implemented in the realm of systematics. But these methods need not be focused anachronistically. As I argue below, PEI needs no percolation. It can be applied now. Meanwhile, everyone else can continue to apply the obsolete paradigm and methods. It still pays. Just do not call it evolutionary biology. Call it evolutionary bookkeeping.

\section{Evolution by natural drift: an operationally useful non-Darwinian conceptualization}

PEI is consilient mechanically with the evolutionary theory of ND, described by Maturana and Mpodozis [24]. This theory is among those non-Darwinian theories viewed contemptuously by Darwinian dogmatists (e.g., [102]), if it they dignify its existence at all. ND maintains several premises that might be described as lemmas:

1. The only constraint on organismal survival/evolution is death. Any organism that is viable theoretically can live and reproduce, and its germline can continue to evolve.

2. Organismal survival/evolution is determined causa sui and does not owe to environmental stimuli. In other words, it is not the stimulus that determines organismal fate, but itself the organism's response, i.e., to survive or die.

3. Organismal survival/evolution owes to no particular trait, but to its entire ontogenetic phenotype, the continuous historical function of all phenotypes expressed during its existence. No phenotype considered favorable at a later stage of life counteracts the presumed defects of an earlier one, and none act retroactively to restore life to a dead organism.

4. As organisms diverge not only genetically, but also spatiotemporally, so do the contingencies for their continued existence. Consequently, statistical equivalency of organisms/traits for comparative analytical purposes decreases.

5. Statistical correlations between particular traits and organismal survival/evolution might exist, yet such correlations do not explain organismal survival/evolution, nor the origin of those traits. 
Hershkovitz

Montiaceae Ecological Evolution

PEI reflects deterministic evolution as conceived in ND, not NS. NS conceives of organismal evolution determined by nonliving (inanimate) forces, often the environment, but including even traits of the organism (e.g., ploidy level or life history). This can be termed Darwinian determinism. This contrasts with evolution conceived as a purely stochastic, hence indeterministic, process [96, 103], as in purely neutral theory.

In practice, the statistical NS paradigm, which includes PhCA, tests deterministic evolutionary hypotheses not mechanistically, but probabilistically. This might seem to be a contradiction in terms, because if evolution is deterministic, it is not a function of probability [103]. But the paradigm only uses stochastic evolutionary null models to test Darwinian deterministic hypotheses [16, 25]. Thus, this stochasticity is contrived for purely epistemic purposes in order to perform epistemologically constrained quantitative induction [103]. Rejection of the stochastic null model is supposed to render highly probable the proposed deterministic hypotheses. At least for linear processes, whether or not the underlying process is truly deterministic is unimportant. Highly probable outcomes axiomatically include absolutely determined ones [103].

Determinism of PEI reflects that of ND theory [24]. Here, the determinism function is assigned tautologically to where it belongs -- the living organism - and to no nonliving factor. In particular, the course of evolution ultimately is determined by the survival and reproduction of organisms. Environmental/organismal traits might challenge or influence the course of evolution, but they do not per se determine it. As long as the organism persists, its fitness remains absolute, not relative. If it dies, regardless of the presumed relative fitness associated with its phenotype, it is out of the game. Thus, both ND and NS theories are deterministic, but they differ in appealing to, respectively, organismal and Darwinian determinism. Parenthetically, the denomination "natural drift" was intended to elicit comparison between the deterministic Darwinian NS and indeterministic genetic drift, and not the contrast between organismal and Darwinian and determinism. ND and PEI both accommodate indeterministic intervention, but PEI appeals explicitly to determinism inherent in chaos theory (see below).

As suggested by its denomination, ND represents not merely an evolutionary mechanism as alternative but coexisting with NS (like genetic drift), but an explicit rejection of NS. Notably, it is not merely consistent with Pigliucci's [109] definition of NS, it is its very incarnation. Its empirically corroborated theoretical prediction is that organisms need to be just "good enough" to survive/reproduce. This is precisely its argument against the necessity, if not existence, of selective filtering.

Special mention must be made of the case of various forms of organismal co-evolution, including socalled kin selection. It can be said that the organisms unilaterally or mutually select, and it is natural, so it is NS. But ND offers a different interpretation. According to ND, the organism determines its fate, and this is nowhere more evident than in the case of organisms selecting other organisms. But this does not constitute "being" selected, because such choices do not themselves determine organismal fate. This depends not on whether the 
Hershkovitz

Montiaceae Ecological Evolution

1 choice was optimal, but merely "good enough." This is ND, or "Pigliuccian selection," not Darwinian NS.

2 However, this may lead to a more organized/obligate coevolutionary relation. Here, there occurs a hierarchical

3 state transcendence, where historically independent organisms behave like single organisms and codetermine their

4 fate accordingly. Accounting for this transcendence, the ND mechanism remains.

\section{Idiosyncraticity of evolution: of chaos, determinism, and stochasticity}

7

Nonlinear chaotic functions render moot the question of evolutionary determinism versus indeterminism, and render untenable the probabilistic adaptationist heuristic. Chaotic mathematical functions, which can model both population and phylogenetic evolution, are fully deterministic [120-123]. By definition, chaotic functions are absolutely orthogenetic and, effectively, infinite-order Markov processes. But, unless the exact function and initial conditions are known, their course and outcomes cannot be calculated. Axiomatically, once underway, the function and initial conditions cannot be calculated, either. Infinitesimal differences in initial conditions radically alter the course of chaotic functions,

More importantly, statistics are of no use in predicting the course of chaotic functions. Even knowledge of exact initial conditions does not improve their statistical predictability. As a chaotic process proceeds, the initial conditions become irrelevant to its incremental evolution [103, 118].

Evolution manifests characteristics of mathematical chaotic functions. These include developmental and evolutionary self-organization and fractal (self-similarity) qualities and consequent emergent properties [118123]. However, these chaotic signatures do not implicate that evolution conforms to a particular chaotic mathematical function. In fact, such seems unlikely, because chaotic function patterns emerge without stochastic intervention/interference. Evolution evidently is intervened/influenced by stochastic processes both within and external to organisms (reviewed in [95]). Thus, evolution can be characterized as a nonlinear and poorly or not predictable process bearing intriguing similarities to chaotic functions. Nothing short of a mathematical "God function" could explain it deterministically or render it predictable.

Idiosyncraticity is not a mathematical function. Formally, it can be defined as the quality of the outcome of a process combining both the unpredictability and deterministic pattern-generating characteristics of a chaotic process while not excluding the effects of stochastic perturbation. This obviates the debate over evolutionary determinism versus stochasticity. The latter debate restricts to "either/or" without considering the possibility of "neither." Accordingly, probabilistic analysis does not improve predictability of an idiosyncratic process or reduce its idiosyncraticity. Note that idiosyncraticity does not apply to a purely chaotic function. Although unpredictable, a chaotic function is determinate, therefore not idiosyncratic. Idiosyncraticity is introduced when it is perturbed. 
Hershkovitz

Montiaceae Ecological Evolution

Note also that the definition of idiosyncraticity refers to the outcome of the evolutionary process and does not attempt to define precisely the process itself. It deduces from empirically observable local/global outcomes the notion that, whatever is the process, it must generate idiosyncraticity. Idiosyncraticity thus characterizes both unique/rare and apparently patterned/frequent outcomes of the process. Thus, Darwinist determinism is not required to explain the latter. Under PEI, patterned evolutionary outcomes might be explained by default as consequence of local chaotic self-similarity in a broader historical context that has generated both self-similarity and nonsimilarity. Self-similarity, in turn, is consequent to the relation between chaos and orthogenesis. However, it is possible also that an apparent pattern is a statistical illusion contrived methodologically, as in [3].

If Darwinian determinism exists at all, the criterion for its detection must discount organismal determinism per ND. Organismal determinism can be compared to a transitory value in a chaotic evolutionary function. The subsequent state is unpredictable, yet it is determined historically (and sensitive to its initial value). The trajectory might be perturbed stochastically by a Darwinian factor, but this factor only influences the trajectory. It does not determine it. This is determined by the organism: whether it lives or dies, and, in the former instance, its mechanism of adaptation to perturbation. This, in turn, returns to its evolutionary history. Its adaptability (or evolvability; see below) is an historically cumulative function. But the response to perturbation is determined by the organism and not per se by the Darwinian factor. However, if the Darwinian factor is historically recurrent (e.g., temperature shifts), the organisms' adaptability/evolvability may reflect learning [95]. An outstanding question is the degree to which historical reconstructions can distinguish stochastic perturbation effects from natural chaotic jump effects. But I suggest that this will be possible by examining all lines of evidence, e.g., from historical biogeography to developmental genetics.

But even if evolution is not mathematically a chaotic function, the existence of the latter bears on evolutionary analysis. In particular, chaotic functions demonstrate that evolutionary processes generating apparently regular patterns can be nonetheless nonlinear, intrinsically deterministic, and unpredictable. This is significant, because the statistical paradigm of evolutionary analysis rests on the plausibility and accuracy of predictable linear functions, and often simplistic ones at that. For analytical purposes, evidently nonlinear evolutionary processes sometimes are approximated by paralinear functions that linearly transform nonlinearity for the purpose of statistical analysis. These include relaxed-clock methods for dating divergences in molecular phylogenies. Chaos theory demonstrates that such approximations may be locally accurate but globally ineffective or erroneous. OE [3] and Smith et al. [12] did apply certain methods that statistically detect localized nonlinear shifts in otherwise linearly-modeled evolution, but they did not appreciate the significance of such detection in terms of chaos-like evolutionary behavior (see below). 
Hershkovitz

Montiaceae Ecological Evolution

DNA-based phylogenetic reconstruction helps clarify the preceding discussion. Most current molecular phylogenetic reconstructions, including those of Montiaceae [5], model DNA sequence evolution as a linear stationary (time-reversible) stochastic process of base substitution. Other sorts of DNA mutations usually are ignored (e.g., methylation and insertions/deletions). Initial (ancestral) conditions are approximated statistically based on alignment of modern DNA sequences. The phylogenetic tree emerges as the branching pattern that, via genetic inheritance, most likely/probably connects the modern sequences with the approximated ancestor given the estimated model. But if DNA sequence evolution were a chaotic process, this approach would be invalid: the process would be nonlinear, the initial conditions could not be estimated reliably, and, even if initial conditions were known, the phylogeny could not be estimated reliably statistically.

However, even if not mathematically chaotic, DNA sequence evolution manifestly is not a linear stationary process, as demonstrated for Montiaceae [5]. It also manifests chaos-like behavior. Unfaithfulness of DNA replication, even if stochastic, creates a fractalization process. The process itself yields base substitution and other mutations (e.g., [124]; ignored in statistical phylogenetic estimation) that alter the functionality of the sequence and influence the succeeding pattern of base substitution. Genome evolution itself manifestly is selforganizing and yields emergent properties. These are properties of chaotic systems.

If DNA sequence evolution is chaos-like rather than purely stochastic, probabilistic phylogenetic analysis should yield statistical anomalies. This appears to be the case. Probability theory dictates that estimation precision and accuracy increases with increasing sample size and converges on the true value of the estimated parameter. As discussed in [5], increasing DNA sampling evidently reduced certainty of Montiaceae phylogenetic estimation at several nodes. This example is not isolated [19]. Unfortunately, phylogenetic analysts in practice rarely challenge the fundamental assumption of stochasticity imposed by standard methods, nor search for or explain statistical anomalies. Admittedly, such anomalies cannot be attributed to chaos without fitting to a mathematical chaotic function (cf. [120]). Given the complexity of evolution, such a function would be difficult or impossible to derive.

But idiosyncraticity can be detected and modeled. Bayesian estimation methods have rendered computationally feasible the incorporation of dynamical (nonstationary) models in evolutionary analysis. These, for all of their faults, at least facilitate heuristic detection of parameter rate changes during phylogeny, including rates of phylogenetic diversification as analyzed in [3, 12]. But these analyses failed to note apparent idiosyncraticity discussed narratively in this work. One approach (applied in [3]) models Lévy or "jump" processes during the course of phylogeny (e.g., [20,21]). These, along with methods to detect statistical outliers and fat-tailed probability density functions, can aid in describing an idiosyncratic evolutionary history and facilitate its evolutionary analysis. But computational sophistication may not be necessary. I detected purely by inspection the idiosyncraticity described narratively in this work and in [5]. 
Hershkovitz

Montiaceae Ecological Evolution

There are caveats to statistical approaches to detection of evolutionary nonlinearity. One is that tests of Lévy and other nonlinear events themselves appeal to stochasticity. Jumps are not determined locally, as in a chaotic, chaos-like, or idiosyncratic process. In practice, they are diagnosed according to their Bayesian-optimized stochastic probability distribution, in turn based on posteriorly estimated prior probabilities (i.e., approximate and not exact initial conditions). Another caveat is that, in practice, Bayesian estimation methods have been shown to be systemically overconfident and even to converge on high probabilities of zero-probability events (e.g. [125, 126]). Adding parametric complexity to Bayesian models exacerbates this problem, because each (latent) parameter is modeled as a stochastic process. Finally the application of the jump and multi-rate tests in, e.g., [3], is essentially as a correction for process nonstationarity. Nonlinear behavior is recognized only if it is "statistically significant." Once corrected for, the PhCA proceeds otherwise under an assumption of process stationarity. Under PEI, a different approach is advocated (see below).

PEI sheds new light on an old debate, viz., the applicability of ML versus MP in phylogenetic and evolutionary reconstruction, as exemplified in $[16,127]$. MP is defended on philosophical grounds, while ML counterarguments appeal to both philosophy and empirical evidence. In particular, it is proposed, based on misleading evidence, that ML yields more accurate reconstructions and is more robust than MP to violations of its assumptions. This evidence is based on phylogenies simulated under linear stochastic models that ML presumes. An erroneous ML model may recover such a phylogeny correctly, because its error is correspondingly linear. Also, comparisons of the performance of ML and MP on such simulated phylogenies tend to focus on single nodes and not each node on large complex trees. In practice, this is unrealistic. Improved accuracy at one node might be at the expense of accuracy at another.

At the same time, PEI partially explains the functionality of ML in practice. Statistical analysis likely would show significant fit of a localized chaotic oscillation to a nonrandom linear stochastic model, and that model likely would reconstruct much of the oscillatory history. Whether such a process would lead ML to outperform MP is not clear. But it does demonstrate that changes along adjacent branches in a phylogenetic tree may be correlated, and that statistical models derived from these correlations indeed might recover phylogeny accurately. While this does not guarantee performance of ML generally, it does provide a theoretical justification for its application.

In any case, as discussed in [5], the failure of ML models in the long run owes to evolutionary idiosyncraticity, which makes organisms different, not the same. With increasing sampling, ML models might appear to be estimated more precisely, but this is an illusion. Not easily detectable statistically, substitution dynamics along individual branches should become different from adjacent branches and do so in nonlinear and unpredictable ways. Because MP is agnostic towards substitution model, it may perform better than ML at some nodes and worse at others. PEI thus provides a theoretical argument corroborating the epistemological argument in favor of MP in both phylogenetic analysis and PhCA. The point might seem moot, given that ML and ML- 
Hershkovitz

Montiaceae Ecological Evolution

based Bayesian methods now dominate in phylogenetic practice. But as I discussed above, this convention reflects not epistemology, but pragmatism. ML is the method that is prescribed institutionally and best facilitated operationally, and this convention traces its history back to a statistical Darwinist paradigm that persists for reasons other than scientific ones.

\section{Idiosyncraticity: perspectives from phylogeography}

Phylogeographic interpretation of Montiaceae and other Portulacineae disjunct distributions was reviewed in [5] in the context of general phylogeographic notions. Even though Montiaceae and Portulacineae distributions conform to patterns of ancient land arrangements, it was concluded that trans-maritime disjunctions owed entirely to recent long distance dispersal (LDD). Given that Montiaceae possess no morphological specialization facilitating LDD, this conclusion yielded two axioms. One, proposed in [128], was that LDD has an intrinsic high likelihood and therefore merits no penalty as an explanation for trans-maritime phytogeographic patterns. The other is that the likelihood of LDD rendered its plausibility even in explaining terrestrial disjunctions. The first axiom, at least, has been substantially corroborated by phylogenetic studies of numerous lineages.

The phylogeographic conclusions above had been framed in probabilistic terms, and indeed multiparametric probabilistic approaches to phylogeography have been proposed more recently [129]. These endeavor to take into consideration every conceivable factor that might have impacted the phylogeographic history of species since their localized origin and throughout the development of their modern distributions. But in [5], I both welcomed and criticized this approach, especially for its reliance on statistics, and especially Bayesian estimation. In particular, idiosyncrasy cannot be predicted statistically.

This conclusion is supported, oddly enough, by statistical modeling of phylogeographic dispersal [130]. "Standard" dispersal mechanisms of organisms yield approximately linear dispersal patterns, where the dispersal distance is a function of intrinsic (standard) dispersability mechanisms/adaptations, whether that distance is short or long. The probability of dispersal distance increases linearly with distance and this distance does not increase with increasing time. This yields a predictable geographic range that might be affected otherwise by likewise "standard" ecological factors that impact survival of the organism in its potential standard dispersal range.

However, the theoretical prediction for "nonstandard" dispersal mechanisms is another matter. A nonstandard mechanism may be any historical occurrence by which the organism disperses by other than its adapted means. The relation between intrinsic dispersability and distance, and between achieved distance and time, are fat-tailed. The do not trend linearly to zero. The distance is theoretically limitless, and the probability that nonstandard distance will be achieved accumulates with time, in relation to the unconstrained probability of an unpredictable historical occurrence. And this provides insight into how, over the course of millions of years, plants with no apparent dispersal adaptation achieve LDD with high likelihood. This principle can be extended to 
Hershkovitz

Montiaceae Ecological Evolution

include rare ecological occurrences and even rare mutations. For example, establishment of a species in a remote location might be improbable because of competition in that location. But an unpredictable ecological disaster might render more likely establishment of exotic arrivals [130].

The patterns predicted by these results are bimodal phylogeographic histories. Standard events yield standard ranges. These are fractal-like, reflecting self-similarity in distribution. Offspring are not identical to their parents or to each other. But their minor dissimilarities are not patterned. This is fractalization consequent to reproduction. Likewise, offspring cannot occupy the exact same spatiotemporal location of their parents, but will generally occupy a very similar one. But the dissimilarities of these new locations likewise are unpatterned, so that no two are exactly alike. This also is a fractal pattern. Both patterns are consequent to a chaos-like process. Both chaotic jumps and perturbation of this process can yield jumps into locations normally not accessible to that species, as well as unique offspring that are capable of jumping into nonstandard locations. In other words, the phylogeographic process conforms to PEI.

\section{Idiosyncraticity: Evidence from NS experiments}

Idiosyncraticity of evolution can be appreciated in view of numerous experimental results, but I will review here only two. Travisano et al. [131, 132] found that monophyletic bacterial clones cultivated separately under identical and nominally suboptimal media all exhibited initially reduced fitness but later recuperated. However, while the fitness of phenotypes converged, the genotypes were different, i.e., different mutation sets achieved the same phenotypic effect. But the authors interpreted the results in terms of Darwinian evolutionary determinism (the effect of the stressful media on bacterial evolution), whereas they demonstrated evolutionary idiosyncraticity. In particular, even though subjected to identical conditions, the clones actually diverged and diversified idiosyncratically. Their evolved phenotypic similarity under the experimental conditions could not have been anything other than coincidental. The suboptimal media did not cause the clones to adapt. They were adapted to begin with, otherwise they would have died. Their subsequent evolutionary trajectories were not and cannot be the same.

Notably, evolutionary idiosyncraticity emerged unrecognized in an experiment optimized to conform to the premise of the Darwinian statistical paradigm. In particular, the paradigm minimizes the importance of hidden/unmeasured variables that yield unexplainable experimental variance and error. Using bacterial cultures maximizes population size and minimizes environmental variation. But this strategy, in turn, renders impossible anything but statistical characterization and analysis of the fate of individuals. Rare genotypes/phenotypes in the original colony were undetectable. Moreover, while uniformity of conditions seems advantageous statistically, it is unrealistic ecologically. And even if uniform conditions were maintained indefinitely, the fitness of a particular genotype is transitory, unless no other genotypes are present and God turns off evolution. 
Hershkovitz

Montiaceae Ecological Evolution

An ND perspective would interpret the results differently. Evidently all colonies originally contained one or more individuals (up to and including all of them) adapted sufficiently to the nominally suboptimal conditions. Nonlethality of these conditions was established experimentally beforehand, which is unrealistic in natural evolution. Subsequent statistical increase in clone fitness owes to the capacity and propensity of evolution to increase organismal fitness in its milieu. In other words, it is determined by the organism and not the milieu.

Evidence for the ND interpretation and PEI emerges from other experimental evidence using bacteria [133]. Here, under constant conditions, initially less fit genotypes eventually could achieve higher fitness than initially more fit genotypes. The authors attributed the finding to higher evolvability of the initially less fit genotype [133]. Evolvability is supposed to correlate with longer-term fitness and, under some circumstances, may be inherently higher among initially less fit individuals $[95,134,137]$. The key observation remains the ability of offspring of less fit individuals to eventually outperform those of the more fit under constant conditions, which supports the notion that fitness is determined by (trajectory of) the organism and not the conditions.

The significance with respect to PEI is that the experimental results above support a chaos-like model of evolution and conflict with premises of the Darwinist statistical paradigm. The latter conceives of evolution as a linear displacement of the phenotypic fitness curve. Better phenotypes reproduce more, such that fitness of offspring of individuals on one side of the curve decreases as that of those on the other side increases. The experimental results of [133] indicate that offspring jump to new fitness curve values such that, over time, the correlation between current and ancestral phenotypic fitness values becomes nonlinear and eventually unpredictable. This is exactly a property of chaotic attraction functions [123]. This suggests that what is perceived as a biological population may be conceived in terms of the population of a chaotic attractor basin. There are many paths to instantaneous optimality.

However, as noted, biological evolution is not known to conform to a particular chaotic function, and probably it does not. The properties of chaotic functions are self-generated and emerge under conditions of kinetic constancy (e.g., growth rate) and without external intervention/perturbation. This is why evolution is characterized better as a chaos-like process whose behavior is idiosyncratic.

\section{Idiosyncraticity: evidence from PhCA of Montiaceae ecological evolution}

The significance of this discussion in the present context is that PEI, if correct, contradicts a principle of prevailing PhCA practice. PhCA seeks to induce macroevolutionary determinism in the framework of epistemic indeterminism, i.e., stochastic evolutionary models. As with DNA phylogenetic estimation, initial parameter values are estimated using current values. The approach is invalid if evolution is chaotic, and probably invalid if evolution is idiosyncratic. The distinctions between chaos and stochasticity and idiosyncraticity are subtle, but not trivial. To paraphrase Lorenz, the patriarch of chaos theory, under chaos, the approximate past does not predict 
Hershkovitz

Montiaceae Ecological Evolution

the approximate (much less exact) present [119]. Presumably this applies also to idiosyncraticity, but idiosyncraticity includes a stochastic component.

PEI bears on ecological evolutionary interpretation of Montiaceae. The PhCA investigations analyzed here conform to the Darwinist statistical paradigm. This posits that Darwinian evolutionary determination (i.e., selection or adaptiveness) is induced by detecting departures from random trait distributions. As noted, PhCA differs from conventional population-level analyses in that the individuals are taxa that might be statistically equivalent from a cladistic standpoint but are nonequivalent by every other tangible measure. The stochastic model parameters and initial conditions themselves are induced rather than measured empirically, and the analyses extrapolate across not single observable, but tens of millions of unobserved generations.

The approach to PhCA is as valid as its premise. Its premise is that evolution proceeds stochastically (a random walk) except as determined by particular inanimate forces/constraints. Nonrandom evolutionary patterns identify these forces/constraints or, contrapositively, the hypothetical forces/constraints can be identified by the patterns they are believed to determine. Meanwhile, organisms are conceived as passive actors in this process. The validity of the premise, however, does not validate the technical assumptions or accuracy of particular PhCAs. But if the premise is valid, the approach itself is valid and should facilitate accurate macroevolutionary interpretation.

Therein lay the problem. Indeed, PhCA corrects for phylogenetic nonindependence in statistical analysis of macroevolutionary causation. But the validity of statistical induction of macroevolutionary causation was problematic, with or without such correction. Statistical analysis of macroevolutionary patterns and correlations are useful for heuristic purposes. But as explanations in themselves, correlations, corrected or not for phylogeny, are probabilized Panglossisms [26]. Indeed, PhCA has reincarnated and reinvigorated classical quasi-logical pseudoscientific Darwinian debates as arcane and byzantine as whether the virtue of a proverbial light beer owes to its "great taste" or (literally in the case of nonentire leaves [136]) its being "less filling," This underscores that, contrary to what [112] might intimate, the popular misconception of evolution as an optimizing process is an orthodoxy of the mainstream Darwinian evolutionary biology establishment and proselytized nowhere else.

Consilient with [16], theoretical and empirical evidence for idiosyncratic evolution invalidates stochastic null models commonly applied in PhCA, both objectively and epistemologically. Such a process can generate patterns whose correlations may be decoupled historically from cause. Instantaneous phenotypic/genotypic frequencies under such a process are not reliable indicators of those of the phylogenetic past or future. The unpredictability of the process owes to the determinacy intrinsic to organisms. As long as organisms survive, their instantaneous relative fitness does not predict future lineage proliferation or diversification. Hence patterns of phylogenetic correlations can change markedly.

Perhaps ironically, idiosyncraticity manifests in OE's [3] own interpretations of their PhCA results. They posit that: (i) Montiaceae depart from other Portulacineae clades in their ecological and morphological 
Hershkovitz

Montiaceae Ecological Evolution

1 phylogenetic lability and evolutionary dynamics; (ii) the inferred "connection of life history and climate

2 variability is specific to Montiaceae;" and (iii) other Portulacineae clades such as cacti "have their own lineage-

3 specific means of occupying a wide range of temperature regimes." These assertions effectively betray weakness

4 of the principal assumption underlying PhCA, which is that phylogeny renders trait evolution predictable.

If, as [3] assert, lineage specific trait evolution manifests among Portulacineae, it should manifest as well

among Montiaceae -- within its clades, species, and populations. Narrative analyses above demonstrate manifold that it does. Some Montiaceae taxa have traits not predicted by observed correlations, e.g., warm-adapted perennials. But manifestly they are fit. Also, narrative analysis demonstrates that the statistical tendencies estimated for Montiaceae collectively do not hold for particular taxa. For example, polyploid Lewisiopsis and Hectorelleae did not diversify phylogenetically/ecologically as expected.

The monotypic and geographically restricted Lewisiopsis is notable: it possesses the growth form and genomic traits predicated to have promoted Montiaceae diversification. It occurs near the geographical and ecological epicenter of Montieae diversity, a heterogeneous landscape that itself is supposed to have facilitated adaptive niche opportunism. Moreover, evidently it is a product of hybridization, another reputed niche diversification catalyst. And yet, there it remains, like Excalibur, alone, unmoved from its Cascade bedrock, unchanged since its internment. Metaphorically, Lewisiopsis is like a presumptively heads-biased coin that has flipped tails successively for ten million years. This suggests a flaw in statistical assumptions, and it evidences evolutionary idiosyncraticity. This and other presented evidence also demonstrate that, under other historical circumstances, calculated correlations between life history, genome duplication, and ecological diversification might have been null or reversed.

Lewisiopsis and other statistical aberrations highlighted here present no difficulty to PEI. Logistic mapping of chaotic attraction functions [123] demonstrates that nearest map neighbors can derive from divergent functional evolutionary trajectories and, contrapositively, similar evolutionary trajectories need not converge to nearby map loci. PEI, which presumes chaos-like evolution, similarly permits unperturbed abrupt divergent evolutionary trajectories of lineages sharing evolutionary and ecological histories. It also suggests that convergence of trajectories need not reflect shared history. approaches appear to anticipate, hence also pursue, evolutionary lawfulness in biodiversity patterning. Note that the very invocation of statistical methods idealizes a coin-likeness of organisms -- individuals, species, and even phyla. This cannot help but introduce systematic determination bias, because the epistemology itself justifies, 
Hershkovitz

Montiaceae Ecological Evolution

even obliges weighting similarities over differences. Thus, the approach imposes a degree of the lawfulness that it seeks to discover.

The current PhCA approach first selects phenomena and data precisely for their predisposition to quantitative inductive analysis. Then, parameters and parameter states tend to be contrived for statistical simplicity. Parameter values often are averaged, which is legitimate only to the degree that such averages indeed reflect optimal or true parameter values, and that deviant values are suboptimal or sampling errors. Under PEI, all observed parameter values are significant regardless of their frequency.

Often, PhCA applies Bayesian methods misleadingly. As discussed in [5], Bayesian estimation in phylogenetics is essentially an algorithmic tool. It renders computationally feasible otherwise computationally unfeasible complex likelihood analyses. Conventionally, with each subsequent empirical observation, Bayesian PPs iteratively improve on estimated prior probabilities. But prior probability optimization in Bayesian phylogenetic estimation is achieved not empirically, but algorithmically via iterative observation of the same set of posterior observations. For this and other reasons [5], Bayesian PhCA is vulnerable to be a form of "induction on steroids."

PEI suggests that the quantitative precision in PhCA, often involving intricate layers of statistical machinations, cannot help but be illusory. Ultimately, the approach appeals to, even if only for epistemic purposes, a notion of coin-likeness of organisms and taxa. But organisms and, much more so, taxa could not be more different from coins, which are minted to be asymptotically identical. Evolution (teleologically speaking) axiomatically mints organisms to be precisely and asymptotically not only different, but different from anything thus far evolved. This innovative capacity is what makes evolution interesting. Otherwise, we would not study evolution, nor be here to study it. Thus, increasing phylogenetic sample size inherently causes statistical inconsistency, or convergence on error. This may be a property of statistical evolutionary analysis generally [23].

An aggravating artifact of the statistical paradigm, noted by no less than Sewell Wright, is a tendency to interpret (or predicate) statistical results as resolute rather than descriptive [103]. Widely applied statistical methods do not merely marginalize nonconforming observations, they effectively obligate and justify their marginalization. While statistical researchers often fastidiously detail their analytical protocols, generally they do not explain or justify in the first place the epistemological applicability/adequacy of the method in an evolutionary biological context. As explained above, this reflects the historical entrenchment of the statistical paradigm in evolutionary biology combined with technological advances (including software and databases) that have facilitated methodological implementation. Consequently, much of the body of empirical evolutionary biology literature is characterized better as technical reporting rather than science [137].

Notably, epistemological issues such as those above (and many others) have been raised (for many decades) in the context of Darwinian population genetic research, where evolution can be observed (more) empirically/experimentally. Empirical/experimental evidence discussed above validates these epistemological 
Hershkovitz

Montiaceae Ecological Evolution

concerns. It cannot be excessively reiterated that PhCA methods, including those applied in Montiaceae research, extrapolate the Darwinian statistical paradigm to the phylogenetic scale, in which the past and future cannot be observed or manipulated. And, as described narratively for Montiaceae, nonconforming observations encompass not merely individuals or population subsamples, but entire species or clades.

PEI suggests that PhCA hypotheses are tested better against an idiosyncratic rather than a linear stochastic default. Mathematically, this is impossible currently (though not inconceivable), given the lack of a mathematical model of idiosyncraticity. But this is not as problematic as it appears. Idiosyncraticity is an epistemological model validated in empirical observation of organismal determination and nonlinearity of evolutionary outcomes. It must be reemphasized that stochastic models applied in evolutionary biology likewise are epistemic. Notwithstanding their quantitative precision, their objectivity roots in statistical and not biological, theory. No statistical procedure nor computer program nor CPU can calculate its way out of this conundrum. Statistics do not, in any case, adjudicate autonomously the validity or accuracy of model assumptions, analyzed parameters, or parameter values.

It is critical to recall that a chaotic process is not a stochastic process. Unperturbed, its outcome is neither predictable, nor random, yet it is determined exactly. Idiosyncraticity is conceived in terms of a stochastically perturbed chaos-like process. Thus, while its outcome is not determined exactly, the outcome distribution is not the same as nor linearly related to the outcome distribution of a linearly parameterized stochastic process. But while existing statistical methods can detect significant nonlinearity in evolutionary processes, the Darwinian paradigm presumes that the failure to detect significant nonlinearity validates the assumption of linear stochasticity. Under the PEI criterion, this assumption is unsupported and untenable. There is no evidence that biological evolution ever is a linear process, not at the population, nor phylogenetic level.

However, in the context of PEI, existing PhCA methods can be applied heuristically. This is not a problem as long as quantitative results are interpreted not resolutely, but merely as biased/nuanced statistical descriptions. The key is to appreciate that PEI predicts exceptions to any statistical generalization, and that quantitative propensities in evolution are transitory. Thus, rather than rounding off values, forcing conformation, and discarding exceptions into the dustbin of residual variance, the exceptions must be sought and their exceptionality highlighted and valued as discoveries and pursued accordingly.

Conceptually, a PEI-based analysis is analogous to searching for new biological species. New species are discovered while searching among known, hence axiomatically statistically predicted species. But known species do not predict unknown species. New species are recognized by their idiosyncrasy. Supposed phylogenetic patterns and correlations, alternatively, better are interpreted as methodologically and conceptually canalized epiphenomena. They essentially are higher taxon descriptions, e.g., for Montiaceae, "perennials often rosettiformcaudiciform hemicryptophytes of cooler environments.....but sometimes not." 
Hershkovitz

Montiaceae Ecological Evolution

Another approach is shotgun application of different PhCA methods in order to look for the patterning conflicts that they might reveal. Lawful evolution predicts that differently theoretically-justified methods should discover the same "reality" and converge on the same explanations. This is the principle of consilience [138]. Methodologically-induced conflicts suggest that one or the other or both results are analytical artifacts. Unfortunately, application of single methods yields no such conflict, hence yields false confidence in the lawfulness of the patterns they reveal.

For example, fortuitously, [3] applied two very different methods to reconstruct Montiaceae life history evolution. The results diametrically opposed. Narrative analysis revealed considerable accuracy of the rejected results. More significantly, the analysis suggested two critical observations: (i) phylogenetic reconstructive methods do not reconstruct the past as reliably as believed; and (ii) phylogenetic relatedness does not predict evolutionary destiny as reliably as believed. Both observations accord with PEI.

As a generalization, typical null and alternative hypotheses of Darwinian PhCA would benefit from inversion and cross-checking. Null and alternative hypotheses often invoke a criterion of random versus patterned data, respectively. But a randomness criterion is not always appropriate. While randomness of data indeed discredits an alternative deterministic hypothesis, departure from randomness does not support it necessarily [23]. More to the point, a proper null hypothesis embodies the default expectation of the investigator.

Scrutiny of some PhCAs suggests that the explicit or implicit alternative hypothesis is, in reality, the default expectation. For example, Smith et al. [12] begin their conclusion by asserting that polyploidy is "almost certainly among the dominant processes that contribute to major evolutionary events within plant lineages." In rhetorical context, the assertion appears to be an alternative hypothesis, affirmed by rejection of a null model. But given the evidence (see above) and Smith et al.'s [12] own correspondingly cautious interpretation thereof, the assertion reveals itself to be an unrejected prior expectation. It is the null hypothesis. The distinction is not trivial. As an alternative hypothesis, its affirmation requires only nonrandom relations between polyploidy and diversification. As a null hypothesis, it can be rejected given significant departures from the predicted correlation.

PEI provides a more apt null hypothesis. PEI implicates unpredictability, but not randomness or unlawfulness of evolution. PEI conceives the default evolutionary process not as a random walk, but rather an "idiosyncratic walk," with a random walk being improbable. However, in Darwinian practice, rejection of random patterns owes partially to contrived nonrandom constraints. In other words, possible steps are constrained to highest-frequency observed states and not to all observed states, nor unobserved states. Ironically, such contrivances do not afford to evolution its quintessential ability to innovate. At the same time, PEI permits, even obligates, emergence of nonrandom patterns, without implicating Darwinian determinism or other thusly derived deterministic lawful relations (e.g., between life form or polyploidy and ecological diversification) that constrain and therefore render evolution predictable. The null model is that such discernable patterns do not contradict PEI. 
Hershkovitz

Montiaceae Ecological Evolution

PEI also provides an alternative interpretation of evolution in terms of Markov processes. The Darwinian paradigm interprets evolution mainly as a first-order Markov process, whether a random walk or determined one. The paradigm also concedes local residual higher-order influence (orthogenesis), such that, e.g., the ancestral Montiaceae life form yielded similar diversification patterns in all descendants. Evolution so conceived is thus mainly a first-order Markov process with incrementally decreasing influence of nested incrementally higher-order processes. PEI idealizes evolution as a continuous superimposed first- and infinite-order Markov process driven by organisms as they navigate their milieu. The first-order process reflects stochastically-encountered obstacles perturbing an otherwise infinite-order chaos-like Markov process. Apparently statistically significant evolutionary patterns recurring at intermediate phylogenetic levels are interpreted as fractal-like, reflecting the infinite order process and not intermediate-order processes.

A protocol for PEI analysis is to presume idiosyncraticity and then use statistics to evaluate both stochasticity and statistical patterns implicating Darwinian determinism. For example, presumed stochasticity of latent parameters can be explored in order to detect biases that in turn percolate to other levels of the analysis. Apparent statistical patterns can be explored more rigorously to determine if (or how) they might arise without invoking any laws other than those inherent to biological existence and reproduction. Thus, indeterminism and Darwinian or other determinisms can be invoked only after idiosyncraticity has been ruled out thoroughly.

Note that a PEI approach obviates the violations of model assumptions of PhCA and phylogenetic reconstruction articulated here and in [5]. This is because they no longer are "assumptions," i.e., taken to be true. They merely are orientation parameters that explicitly impose a presumed evolutionary lawfulness for epistemic purposes, e.g., a substitution model or Darwinian determinism. Again, this lawfulness is the null and not the alternative hypothesis. What are "truths" in a PEI approach are all observations, including those not predicted by lawfulness. Conventional PhCA explains unpredicted biological truths ad hoc (e.g., "hidden variables," [103]) or as "statistical error." Epistemological error is not suspected. In a PEI approach, violations of statistical assumptions are not problematic. To the contrary, the discovery of assumption violations is precisely the objective.

Thus, unlike a Darwinian determinist model, a failure to reject PEI does not render fruitless the analytical effort. To the contrary, and aside from its value in challenging pervasive evolutionary notions, it facilitates biological discovery. Analysis founded in PEI permits unraveling of mechanisms that appear to yield similar, yet ingeniously unique, evolutionary products. In this way, PEI views biological evolution as analogous to computational hacking. Evolutionary analysis, therefore, is best approached the same way that computer hacking is studies. 
Hershkovitz

Montiaceae Ecological Evolution

In summary, PEI is a useful and powerful concept for biodiversity research. In an informal sense, idiosyncraticity is comprehensible intuitively. But its intuitive definition qualitatively approximates the formal definition described here. Thus, it facilitates quantitative analysis and argument and it motivates analytical rigor. PEI subsumes and integrates -- and predicts -- all empirically-evidenced concepts of nonlinear evolutionary phenomena, spanning the population (e.g., shifting balance, evolvability) to phylogenetic (e.g., punctuated equilibria) scales. It also describes nonstationary molecular evolution, which undermines both phylogenetic reconstruction and molecular dating [5]. And it describes historical phylogeography. PEI is thusly functional because it refers to the outcome of the process that yields it. In this sense, it is a nonmathematical analogy of the Price Equation (see above).

Most important, the process that yields idiosyncratic (and not chaotic or stochastic) outcome seems to correspond to empirical biological reality. NS and its PhCA methodological derivatives approximate biological fantasy forced upon reality. And PEI reality likewise can be explained intuitively, borrowing an analogy from [5]. Observing automobile traffic patterns, one might compare the autos with individuals in a population being selected to move towards a destination and competing among themselves to arrive there in minimal time. But only if one watches a particular stretch of highway. In fact, the autos have different origins and destinations. The origins/destinations might be mostly similar, but not identical, and some are radically distinct, similar to a chaotic function. Stochastic events, whether a statistically predictable disabled vehicle or an unpredictable Boeing 787 landing on the roadway, might perturb the trajectories. But the trajectories are dispersed likewise chaotically, and not linearly. The single most important observation is that, as in ND [24] and not NS, ultimately the drivers, and not the traffic pattern or the origin or the destination or the perturbations, take the actions that determine their destinies.

In this way, PEI facilitates a priori perception and conceptualization of evolution as nonlinear and unpredictable, even as it is analyzed linearly and probabilistically. It facilitates understanding that evolution itself is not an evolutionary model, and that the utility of models is heuristic [139], but limited [140]. Idiosyncraticity is not a model, but an anti-model or, perhaps, a "model that cannot be modeled." Yet, it can be detected and described by pitting models against each other. Its unpredictability is predictable, model failures are its success, and noise is its signal.

PEI bridges traditional empirical evolutionary research with vanguard theoretical research on chaos and complexity [12]. This is significant because the latter provide evolutionary models more theoretically/empirically realistic than the Darwinian model. In fact, it is from these and other theoretical realms that have emerged empirically-validated criticisms and even rejections of the Darwinian paradigm [95: 4]. However, these theoretical lines of research barely have percolated into empirical evolutionary biology, which has been dominated for 80 years virtually hegemonically and intransigently by the statistical Darwinist paradigm. 
Hershkovitz

Montiaceae Ecological Evolution

A PEI approach provides an avenue for direct collaboration between theoretical and empirical evolutionary biology. The Darwinian approach does not. As described throughout this work, at successive analytical stages, the Darwinian approach selectively sequesters critical observations and smoothes over the remainder. It does not facilitate interpretation under anything other than the Darwinian model. A PEI approach can apply Darwinian methodology in a way that renders singularities more evident. I reiterate that PEI focuses on evolutionary outcomes. It is epistemologically ambiguous/agnostic, and therefore permissive, towards physical evolutionary mechanisms. In this way, empirical information can be presented a way that invites multiple epistemological analyses and interpretations.

PEI also justifies institutional reintegration of the divergent paradigms of quantitative and narrative biodiversity research. It implores correction of the epistemological poverty of the former and institutional marginalization/elimination of the latter. PEI demonstrates that, in evolutionary biology, the sophistication of mathematics cannot overcome descriptive incompleteness, imprecision, and inaccuracy. To the contrary, PEI demonstrates that the abstract reductionism spawned and epistemologically justified by statistical Darwinism cannot help but converge on pseudoscience. Alternatively, the lasting contributions to biodiversity research result from acute natural historical observation and critical description.

As in all of science, the objective of evolution research must be discovery. But discovery lies as much in the realm of the conceptual as it does the material. In particular, it requires escape from that which has been thus conceived. Modern evolutionary research is materialistic. It emphasizes ever more sophisticated computational analysis of ever larger masses of data. But the epistemology bundled into software and data rarely is appreciated. This is why much of the body of modern scientific publication amounts to "technical reports" [137]. Much of empirical phylogenetic and PhCA research probably falls into this category. But not all of it. Gerhold et al. [141] challenged conceptually conventional application of PhCA to the study of ecological community assembly. Perhaps not coincidentally, Ricklefs [142], following decades of prolific ecological community research, challenged the conceptual notion of community. In both cases, the advances are not material, but conceptual. Perhaps also not coincidentally, both advances conform to PEI.

\section{Abbreviations}

MP: maximum parsimony; ML: maximum likelihood; ND: natural drift; NS: natural selection; PhCA: phylogenetic comparative analysis; PEI: principal of evolutionary idiosyncraticity.

\section{Acknowledgements}

Elaboration of the present work would not have been possible except for the generous support and dedicated efforts of Katia Chechelnitzky, Sandra Steuermann, and Gabriela Feldman of Fundación Reshet in Chile (www.reshet.cl). I gratefully acknowledge Mary Endress for helpful review of an earlier version of this 
Hershkovitz

Montiaceae Ecological Evolution

work and suggestions. I also gratefully acknowledge the many Montiaceae researchers who generously shared with me (via their journal publications) their data and interpretations. This article was inspired by the life work of my late father, Philip Hershkovitz, especially [76: 64]: "Evidently, the more extensive the mathematical interventions, the more complicated become the statistical analyses and the more sterile, futile, and unrealistic their results." My enlightenment in evolutionary theory owes to members of the Laboratory of Neuroscience and Biology of Knowledge, University of Chile, to whom, despite their Stalinist orthodoxy, I remain grateful. PEI was conceived and developed while contemplating horse races at Club Hípico de Santiago and Hipódromo Chile (Santiago, Chile) and Valparaiso Sporting Club (Viña del Mar, Chile).

\section{References}

1. Hershkovitz MA. Synopsis of a new taxonomic synthesis of Montiaceae (Portulacineae) based on rational metadata analysis, with critical new insights on historically poorly understood taxa and a reevaluation of historical biogeography. Preprints 2018; 2018080496. https://doi.org/10.20944/preprints201808.0496.v1

2. Hernández-Ledesma P, Berendsohn WG, Borsch T, von Mering S, Akhani H, Arias S, Castañeda-Noa I, Eggli U, Eriksson R, Flores-Olvera H, Fuentes-Bazán S, Kadereit G, Klak KC, Korotkova N, Nyffeler R, Ocampo G, Ochoterena H, Oxelman B, Rabeler RK, Sanchez A, Schlumpberger BO, Uotila O. A taxonomic backbone for the global synthesis of species diversity in the angiosperm order Caryophyllales. Willdenowia 2015;45:281-383. https://doi.org/10.3372/wi.45.45301

3. Ogburn RM, Edwards EJ. Life history lability underlies rapid climate niche evolution in the angiosperm clade Montiaceae. Mol Phylogenet Evol. 2015;92:181-192. https://doi.org/10.1016/j.ympev.2015.06.006

4. Hancock LP, Obbens F, Moore AJ, Thiele K, de Vos JM, West J, Holtum JAM, Edwards EJ. Phylogeny, evolution, and biogeographic history of Calandrinia (Montiaceae). Amer J Bot. 2018;105:1-14. https://doi.org/10.1002/ajb2.1110

5. Hershkovitz MA. Perspectives from Montiaceae (Portulacineae) evolution. I. Phylogeny and phylogeography. Preprints 2018; 201809.0096. https://doi.org/10.20944/preprints201809.0096.v1

6. Hershkovitz MA. Revised circumscription and sectional taxonomy of Calandrinia Kunth and Montiopsis Kuntze (Portulacaceae) with notes on phylogeny of the portulacaceous alliance. Ann Missouri Bot Gard. 1993;80:333-365. https://doi.org/10.2307/2399789

7. Ocampo G, Columbus JT. Molecular phylogenetics, historical biogeography, and chromosome evolution of Portulaca (Portulacaceae). Mol Phylogen Evol. 2012;63:97-112.

8. Carolin RC. A review of the family Portulacaceae. Austral J Bot. 1987;35:383-412. https://doi.org/10.1071/BT9870383

9. Rowley GD. Anacampseros and allied Genera - a reassessment. Bradleya 1994;12:105-112. https://doi.org/10.25223/brad.n12.1994.a8 
Hershkovitz

Montiaceae Ecological Evolution

10. Smith SA, Beaulieu JM. Life history influences rates of climatic niche evolution in flowering plants. Proc R Soc London B. 2009;276:4345-4352. https://doi.org/10.1098/rspb.2009.1176

11. McIntyre PJ. Polyploidy associated with altered and broader ecological niches in the Claytonia perfoliata (Portulacaceae) species complex. Amer J Bot. 2012;99:655-662. https://doi.org/10.3732/ajb.1100466

12. Smith SA, Brown JW, Yang Y, Bruenn R, Drummond CP, Brockington SF, Walker JF, Last N, Douglas NA, Moore M J. Disparity, diversity, and duplications in the Caryophyllales. New Phytol. 2018;217:836854. https://doi.org/10.1111/nph.14772

13. Mouquet N, Devictor V, Meynard CN, Muñoz F, Bersier L-F, Chave J, Coutron P, Dalecky A, Fontaine C, Gravel D, Hardy OJ, Jabot F, Lavergne S, Leibold M, Mouillot D, Münkemüller T, Pavoine S, Prinzing A, Rodrigues ASL, Rohr RP, Thébault E, Thuiller W. Ecophylogenetics: advances and perspectives. Biol Rev. 2012;87:769-785. https://doi.org/10.1111/j.1469-185X.2012.00224.X

14. Pennell MW, Harmon LJ. An integrative view of phylogenetic comparative methods: connections to population genetics, community ecology, and paleobiology. Ann NY Acad Sci. 2013;1289:90-105. https://doi.org/10.1111/nyas.12157

15. Felsenstein J. Phylogenies and the comparative method. Amer Nat. 1985;125:1-15. https://doi.org/10.1086/284325

16. Wenzel JW, Carpenter JM. Comparing methods: adaptive traits and tests of adaptation. In: Eggleton P, Vane-Wright RI, editors. Phylogenetics and Ecology. London: Academic Press; 1994. p. 79-101.

17. Yang Z, Goldman N. Maximum likelihood trees from DNA sequences: a peculiar statistical estimation problem. Syst Biol. 1995;44:384-399. https://doi.org/10.1093/sysbio/44.3.384

18. Podani, J. Different from trees, more than metaphors: branching silhouettes -- corals, cacti, and the oaks. Syst Biol. 2017;66:737-753. https://doi.org/10.1093/sysbio/syx039

19. Pagel M. Inferring the historical patterns of biological evolution. Nature 1999;401:877-884. https://doi.org/10.1038/44766

20. Landis MJ, Joshua G, Schraiber JG, Liang M. Phylogenetic analysis using Lévy processes: finding jumps in the evolution of continuous traits. Syst Biol. 2013;62:193-204. https://doi.org/10.1093/sysbio/sys086

21. Duchen P, Leuenberger C, Szilágyi SM, Harmon L, Eastman J, Schweizer M, Wegmann D. Inference of evolutionary jumps in large phylogenies using Lévy Processes. Syst Biol. 2017;66:950-963. https://doi.org/10.1093/sysbio/syx028

22. Molina-Venegas R, Rodríguez MÁ. Revisiting phylogenetic signal; strong or negligible impacts of polytomies and branch length information? BMC Evol Biol. 2017;17: 53. https://doi.org/10.1186/s12862$\underline{017-0898-y}$

23. Matthen M, Ariew A. Selection and causation. Philos Sci. 2009;76:201-224. https://doi.org/10.1086/648102 
Hershkovitz

Montiaceae Ecological Evolution

24. Maturana HR, Mpodozis J. The origin of species by means of natural drift. Rev Chil Hist Nat. 2000;73:261-310. https://www.researchgate.net/profile/Jorge Mpodozis/publication/262497422 El origen de las especies por medio de la deriva natural/links/0c96053bd4f4696eb5000000.pdf

25. Franz NM, Engel MS. Can higher-level phylogenies of weevils explain their evolutionary success? A critical review. Syst Entemol. 2010:56:531-539. https://doi.org/10.1111/j.1365-3113.2010.00534.x

26. Gould SJ, Lewontin RC. The spandrels of San Marco and the Panglossian paradigm: a critique of the adaptationist programme. Proc R Soc London B. 1979;205:581-598.

27. Stoughton TR, Jolles DD, O'Quinn RL. The western spring beauties, Claytonia lanceolata (Montiaceae): a review and revised taxonomy for California. Syst Bot. 2017;42:283-300.

https://doi.org/10.1002/ajb2.1061

28. Wheeler QD, Raven PH, Wilson EO. Taxonomy: impediment or expedient? Science 2004;303:285. https://doi.org/10.1126/science.303.5656.285

29. Wilson EO. Taxonomy as a fundamental discipline. Phil Trans Roy Soc London B. 2004;359: 739. https://doi.org/10.1098/rstb.2003.1440

30. Agnarsson I, Kuntner M. Taxonomy in a changing world: seeking solutions for a science in crisis. Syst Biol. 2007:56:531-539. https://doi.org/10.1080/10635150701424546

31. Rapini A. Classes or individuals? The paradox of systematics revisited. Stud Hist Phil Biol Biomed Sci. 2004;35:675-695. https://doi.org/10.1016/j.shpsc.2004.09.006

32. Körner C, Hiltbrunner E. The 90 ways to describe temperature. Perspectives Plant Ecol Evol Syst. 2018; 30:16-21. https://doi.org/10.1016/j.ppees.2017.04.004

33. Price T. Phylogeny and evolution of Phemeranthus (Montiaceae) in North American xeric habitats. PhD dissertation, Washington University, St Louis; 2012. [Electronic theses and dissertations, Paper 724 http://openscholarship.wustl.edu/etd/724].

34. Heibl C, Renner SS. Distribution models and a dated phylogeny for Chilean Oxalis Species reveal occupation of new habitats by different lineages, not rapid adaptive radiation. Syst Biol. 2012;61:823834. https://doi.org/10.1093/sysbio/sys034

35. Moreira Muñoz A. Plant geography of Chile: an essay on postmodern biogeography. Berlin: Springer; 2011. https://www.researchgate.net/profile/Andres_Moreira2/publication/240997817_The_Extravagant_Physica 1 Geography of Chile/links/0deec534e6f3ba23d6000000/The-Extravagant-Physical-Geography-ofChile.pdf

36. Wernham HF. Floral evolution, with particular reference to the sympetalous dicotyledons. New Phytol. 1912;11:217-235. https://doi.org/10.1111/j.1469-8137.1912.tb05632.x 
Hershkovitz

Montiaceae Ecological Evolution

37. Sanderson MJ, Hufford L. Homoplasy: the recurrence of similarity in evolution. New York: Academic Press; 1996.

38. Cantino PD. Phylogenetic inference from nonuniversal derived states. Syst Bot. 1985;10:119-122. http://www.jstor.org/stable/2418441

39. O'Quinn RL. Phylogeny, biogeography and evolution of perennation structures in Montieae (Portulacaceae). PhD dissertation, Washington State Univ., Pullman (WA); 2005. https://research.wsulibs.wsu.edu/xmlui/bitstream/handle/2376/297/r oquinn 020905.pdf?sequence=1

40. Friedman J, Rubin MJ. All in good time: Understanding annual and perennial strategies in plants Amer J Bot. 2015;102:497-499. https://doi.org/ 10.3732/ajb.1500062

41. Mathew B. The genus Lewisia. Portland (OR): Timber Press; 1989.

42. Davidson R. Lewisias. Portland (OR): Timber Press; 2000.

43. Barrett SCH, Harder LD, Worley AC. The comparative biology of pollination and mating in flowering plants. Phil Trans R Soc London B. 1996;351:1271-1280. https://doi.org/doi: 10.1098/rstb.1996.0110

44. Eggli U. A synopsis of woody Portulacaceae in Madagascar Adansonia 1997;19:45-59. http://sciencepress.mnhn.fr/sites/default/files/articles/pdf/a1997n1a4.pdf

45. Tank DC, Olmstead RG. From annuals to perennials: phylogeny of subtribe Castillejinae (Orobanchaceae). Amer J Bot. 2008;95:608-625. https://doi.org/10.3732/ajb.2007346

46. Hershkovitz MA. Evolution of western American Portulacaceae in the Andean region. Gayana 2006;63:13-74. http://dx.doi.org/10.4067/S0717-66432006000100002

47. Guilliams CM. Phylogenetic reconstruction, character evolution, and conservation in the genus Calyptridium (Montiaceae). Masters thesis, San Diego State University; 2009. http://www.sci.sdsu.edu/plants/lab/Guilliams2009-ThesisCalyptridium-Montiac-r.pdf

48. Arroyo MTK, Armesto JJ, Villagrán, C. Plant phonological patterns in the high Andean Cordillera of Central Chile. J Ecol. 1981:69:205-233. https://doi.org/10.2307/2259826

49. Hershkovitz MA, Arroyo MTK, Bell C, Hinojosa LF. Phylogeny of Chaetanthera (Asteraceae: Mutisieae) reveals both ancient and recent origins of the high elevation lineages. Mol Phylogen Evol. 2006;41:594-605. https://doi.org/10.1016/j.ympev.2006.05.003

50. Ford[-Werntz] DI. Systematics and evolution of Montiopsis subgenus Montiopsis (Portulacaceae). PhD dissertation, Washington University, St Louis; 1992.

51. Guilliams CM, Miller JM. Calyptridium pygmaeum. In: Jepson Flora Project (editors), Jepson eFlora, Revision 2, 2014. http://ucjeps.berkeley.edu/eflora/eflora display.php?tid=16849, accessed on $27 \mathrm{Sept}$ 2018.

52. GBIF Secretariat. Cistanthe minuscula (Añon) I. Peralta. In: GBIF Backbone Taxonomy; 2017. https://doi.org/10.15468/39omei,accessed via GBIF.org on 26 Sept 2018. 2018-09-26. 
Hershkovitz

Montiaceae Ecological Evolution

53. Hinton WF. Introgression and the evolution of selfing in Calyptridium monospermum (Portulacaceae). Syst Bot. 1976;1:85-90. http://www.jstor.org/stable/2418841

54. Guilliams CM, Simpson MG, Rebman JP. Calyptridium parryi var. martirense (Montiaceae), a new taxon endemic to Sierra de San Pedro Mártir, Baja California, Mexico. Madroño 2011;58:258-266. https://doi.org/10.3120/0024-9637-58.4.258

55. Guilliams CM, Miller JM. Calyptridium parryi var. nevadense. In: Jepson Flora Project, editors. Jepson eFlora Revisión 2; 2014. http://ucjeps.berkeley.edu/eflora/eflora display.php?tid=55523. 2014. Accessed 11 December 2017.

56. West-Eberhard, MJ. Developmental plasticity and evolution. Oxford (UK): Oxford University; 2003.

57. Heidel AJ, Kiefer C, Coupland G, Rose LE. Pinpointing genes underlying annual/perennial transitions with comparative genomics. BMC Genomics 2016;17:921. https://doi.org/10.1186/s12864-016-3274.

58. Kiefer C, Severing E, Karl R, Bergonzi S, Koch M, Tresch A, Coupland G. Divergence of annual and perennial species in the Brassicaceae and the contributions of cis-acting variation at FLC orthologues. Mol Ecol. 2017;26:3437-3457. https://doi.org/10.1111/mec.14084

59. Park J-Y, Kim H, Lee I. Comparative analysis of molecular and physiological traits between perennial Arabidopsis alpina Pajares and annual Arabidopsis thaliana Sy-O. Nature Sci Rep. 2017;7:13348. https://doi.org/10.1038/s41598-017-13606-7

60. Hershkovitz MA. Phylogenetic assessment and revised circumscription of Cistanthe Spach (Portulacaceae). Ann Missouri Bot Gard. 1991;78:1009-1021. http://www.jstor.org/stable/2399741

61. Hershkovitz MA. Leaf morphology of Cistanthe Spach (Portulacaceae). Ann Missouri Bot Gard. 1991;78:1022-1060. http://www.jstor.org/stable/2399742

62. Hinton WF. Systematics of the Calyptridium umbellatum complex (Portulacaceae). Brittonia 1975;27:197-208. https://doi.org/10.2307/2805891

63. Hershkovitz MA, Hogan SB. Lewisia. In: FNA Committee, editors. Flora of North America north of Mexico. Vol. 3. New York: Oxford Univ.; 2004. p. 476-485.

64. Jokerst JD. The vascular plant flora of Table Mountain, Butte County, California. Madroño 1983;30(4) Suppl 1-18. http://www.jstor.org/stable/41426209

65. O’Quinn R[L], Hufford L, Meerow AW. Molecular Systematics of Montieae (Portulacaceae): implications for taxonomy. Biogeogr Ecol Syst Bot. 2005;30:314-331. https://doi.org/10.1600/0363644054223594

66. Miller JM, Chambers KL. Claytonia rubra subsp. rubra. In: Jepson Flora Project, editors. Jepson eFlora. http://ucjeps.berkeley.edu/eflora/eflora display.php?tid=74699. 2012. Accessed 11 December 2017.

67. Miller JM. Claytonia. In: Flora North America Committee (editors), Flora of North America eFlora. http://www.efloras.org/florataxon.aspx?flora id=1\&taxon id=107275, Accessed 27-Sept 2018. 
Hershkovitz

Montiaceae Ecological Evolution

68. Ocampo G. Portulacaceae. In: Lot AM (editor), Plantas acuáticas mexicanas. Vol. 2, Dicotiledóneas. Part 1. Mexico City: Instituto de Biología, Universidad Nacional Autónoma de México: 2017. p. 201-206. https://www.researchgate.net/publication/322993036

69. Les DH. Aquatic dicotyledons of North America: ecology, life-history, and systematics. Boca Raton (FL): CRC; 2017.

70. Ogburn RM, Edwards EJ. The ecological water-use strategies of succulent plants. Advances Bot Res. 2010;55:179-226. https://doi.org/10.1016/S0065-2296(10)55004-3

71. Gibson AC. Photosynthetic organs of desert plants. Bioscience 1998;48:911-920. http://www.jstor.org/stable/1313295 .

72. Herbig A, Kull U. Leaves and ramification. In: Belger F (editor), Natural structures: proceedings of the 2nd international symposium of the Sonderforschungsbereich 230, Vol. 2. Stuttgart: Vorstand. 1992, p. 109-117. http://dx.doi.org/10.18419/opus-2298

73. Christenhusz JM.746. Tropaeolum majus. Curtis’s Bot Mag. 2012;29:331-340. https://doi.org/10.1111/curt.12002

74. Watson J, Flores A. Tropaeolum sect. Chilensia. Curtis's Bot Mag. 2010;27:197-234. https://doi.org/10.1111/j.1467-8748.2010.01709.x

75. Hershkovitz MA, Hernández-Pellicer, Arroyo MTK. Ribosomal DNA evidence for the diversification of Tropaeolum sect. Chilensia (Tropaeolaceae). Pl Syst Evol. 206;260:1-24. https://doi.org/10.1007/s00606$\underline{006-0428-7}$

76. Hershkovitz P. Living New World Monkeys. Chicago: University of Chicago; 1977.

77. Hershkovitz MA. Phylogenetic studies in Centrospermae: a brief appraisal. Taxon 1989;38:602-608. http://www.jstor.org/stable/2419519

78. Dragicevic P. Fair statistical communication in HCI. In: Robertson J, Kaptein M, editor. Modern statistical methods for HCI. Switzerland: Springer; 2016. p. 291-330.

79. Seehausen O. Hybridization and adaptive radiation. TREE 2004;19:198-207. https://doi.org/10.1016/j.tree.2004.01.003

80. Soltis DE, Soltis PS, Tate JA. Advances in the study of polyploidy since Plant Speciation. New Phytol. 2003;161:173-191. https://doi.org/10.1046/j.1469-8137.2003.00948.x

81. Schierenbeck KA, Ellstrand NC. Hybridization and the evolution of invasiveness in plants and other organisms. Biol Invasions 2009;11:1093. https://doi.org/10.1007/s10530-008-9388xDOIhttps://doi.org/10.1007/s10530-008-9388-x

82. Soltis, DS, Visger CL, Soltis PS. The polyploidy revolution then... and now: Stebbins revisited. Amer J Bot. 2014;101:1057-1078. https://doi.org/10.3732/ajb.1400178 
Hershkovitz

Montiaceae Ecological Evolution

83. Soltis, DE, Visger CL, Marchant DB, Soltis PS. Polyploidy: pitfalls and paths to a paradigm. Amer J Bot. 2016;103:1146-1166. https://doi.org/10.3732/ajb.1500501

84. McIntyre PJ. Cytogeography and genome size variation in the Claytonia perfoliata (Portulacaceae) polyploidy complex. Ann Bot. 2012;110:1195-1203. https://doi.org/10.1093/aob/mcs187

85. Miller JM, Chambers KL. Systematics of Claytonia (Portulacaceae). Syst Bot Monogr. 2006;78:1-236. https://doi.org/10.2307/25027952

86. Wagstaff SJ, Hennion FO. Evolution and biogeography of Lyallia and Hectorella (Portulacaceae), geographically isolated sisters from the Southern Hemisphere. Antarctic Sci. 2007;19:417-426. https://doi.org/10.1017/S0954102007000648

87. Barrier M, Baldwin BG, Robichaux RH, Purugganan, MD. Interspecific hybrid ancestry of a plant adaptive radiation: allopolyploidy of the Hawaiian silversword alliance (Asteraceae) inferred from floral homeotic gene duplications. Mol Biol Evol. 1999;16:1105-1113. https://doi.org/10.1093/oxfordjournals.molbev.a026200

88. Stevens PF. Metaphors and typology in the development of botanical systematics 1690-1960, or the art of putting new wine in old bottles. Taxon 1984:33:169-211. https://doi.org/10.2307/1221161

89. Hennig W. Cladistic analysis or cladistic classification?: A reply to Ernst Mayr. Syst Zool. 1975:24:244256. https://doi.org/10.2307/2412765

90. Mayr E, Ashlock PD. Principles of systematic zoology. New York: MacGraw Hill; 1991.

91. Cronquist A. A botanical critique of cladism. Bot Rev. 1987:53:1-52. https://doi.org/10.1007/BF02858181

92. Rodman JE, Oliver MK, Nakamura RR, McClammer Jr JU, Bledsoe AH. A taxonomic analysis and revised classification of Centrospermae. Syst Bot. 1984;9:297-323. http://www.jstor.org/stable/2418610

93. Felsenstein J. Evolutionary trees from DNA sequences: maximum likelihood approach. J Mol Evol. 1981;17:368-376. https://doi.org/10.1007/BF01734359

94. Felsenstein J. Confidence limits on phylogenies: an approach using the bootstrap. Evolution 1985;39:783791. https://doi.org/10.1111/j.1558-5646.1985.tb00420.x

95. Heininger K. Duality of stochasticity and natural selection: a cybernetic evolution theory. WMC Ecology 2015;6(2):WMC004796. https://www.webmedcentral.com/article_view/4804

96. Ulanowicz RE. A Third Window: Natural Life Beyond Newton And Darwin. West Conshohocken (UK) Templeton Foundation; 2009.

97. Salthe SN. Natural selection in relation to complexity. Artificial Life 208;14:363-374. https://doi.org/10.1162/artl.2008.14.3.14309

98. Bak P, Sneppen K. Punctuated equilibrium and criticality in a simple model of evolution. Phys Rev Lett. 1993;71:4083-4086. https://doi.org/10.1103/PhysRevLett.71.4083 
Hershkovitz

Montiaceae Ecological Evolution

99. Davila-Velderrain J, Alvarez-Buylla ER. Bridging the genotype and the phenotype: towards an epigenetic landscape approach to evolutionary systems biology. bioRxiv.org; 2014. http://dx.doi.org/10.1101/004218

100. Koonin EV, Wolf YI. Just how Lamarckian is CRISPR-Cas immunity: the continuum of evolvability mechanisms Biology Direct 2016;11:9. https://doi.org/10.1186/s13062-016-0111-z

101. Frank SA. Natural selection. IV. The Price equation. J Evol Biol. 2012;25:102-1019. https://doi.org/10.1111/j.1420-9101.2012.02498.x

102. Nespolo RF. Evolution by natural selection: more evidence than ever before. Rev Chilena Hist Nat. 203:76:699-716. https://www.uv.mx/personal/tcarmona/files/2010/08/Nespolo-2003.pdf

103. Millstein RL. Is the evolutionary process deterministic or indeterministic? An argument for agnosticism. Presented at the Biennial Meeting of the Philosophy of Science Association, Vancouver, Canada, November 2000. http://hypatia.ss.uci.edu/lps/psa2k/evolutionary-agnosticism.pdf.

104. Gould SJ. Wonderful life: the Burgess Shale and the nature of history. New York: Norton;1989.

105. Daniels G, McGrath D, Cohen DS, Kushel R. Time and punishment. In: Mirken D (producer), Treehouse of Horror V. The Simpsons, Season 6, Episode 109. 1994. Part 1: https://m.youtube.com/watch?v=dUsdMdOmBDw; Part 2: https://m.youtube.com/watch?v=7NwRH$\underline{\text { KbecI }}$

106. Block N, Kitcher P. Misunderstanding Darwin. The Boston Review, March/April 2010. http://bostonreview.net/archives/BR35.2/block_kitcher.php

107. Coyne JA. The improbability pump. The Nation, March 2010. https://www.thenation.com/article/improbability-pump/

108. Futuyma DJ. Two critics without a clue. Science 2010;328:692-693.

109. Pigliucci M. What Darwin Got Wrong by Jerry Fodor and Massimo Piattelli-Palmarini. Philosophy Now $2010 ; 81$. https://philosophynow.org/issues/81/What Darwin Got Wrong by Jerry Fodor and Massimo Piattelli

110. Ruse M. Philosophers rip Darwin. Chronicle of Higher Education, March 2010. https://www.chronicle.com/article/What-Darwins-Doubters-Get/64457

111. Darwin CR. On the origin of species by means of natural selection, or the preservation of favoured races in the struggle for life. London (UK): John Murray. 1859. In: van Wyhe, J. (editor). The complete work of Charles Darwin online (http: //darwin online.org.uk/).

112. Darwin C. The variation of animals and plants under domestication. London (UK): John Murray;1868. In: van Wyhe, J. (editor). The complete work of Charles Darwin online (http: //darwin online.org.uk/).

113. Pigliucci M, Müller GB (editors). The extended synthesis. Cambridge (Massachusetts): MIT Press;2010. 114. Lynch M. The origins of genome architecture. Sunderland,( Massachusetts): Sinauer; 2007. 
Hershkovitz

Montiaceae Ecological Evolution

115. Lindzen RS. Climate alarmism and historical precedents. J Amer Physicians Surgeons 2013;18:70-74. http://www.realdailybuzz.com/rdb.nsf/0/5c6d19746274082485257c22004e6b24/\$file/LINDZEN.pdf

116. Crisp MD, Arroyo MTK, Cook LG, Gandolfo MA, Jordan GJ, McGlone MS, Weston PS, Westoby M, Wilf P, Linder, HP. Phylogenetic biome conservatism on a global scale. Nature 2009;458:754-756. https://doi.org/10.1038/nature07764

117. Guerrero PC, Rosas M, Arroyo MTK, Wiens JJ. Evolutionary lag times and recent origin of the biota of an ancient desert (Atacama-Sechura). Proc Nat Acad Sci USA 2013;110:11469-11474. https://doi.org/10.1073/pnas.1308721110

118. Werndl C. What are the new implications of chaos for unpredictability. Brit J Phil Sci. 2009;60:195-230. https://doi.org/10.1093/bjps/axn053

119. Ferrière R, Fox GA. Chaos and evolution. Trends Ecol Evol. 1995;10:480-485. https://doi.org/10.1016/S0169-5347(00)89194-6

120. Sornette D. Critical phenomena in natural sciences. Berlin, Heidelberg: Springer; 2006.

121. Zuchowski LC. Disentangling complexity from randomness and chaos. Entropy 2012;14:177-212. http://dx.doi.org/10.3390/e14020177

122. Strogatz SH Nonlinear dynamics and chaos. Edition 2.Boca Raton (Florida): CRC;2018.

123. Boeing G. Systems visual analysis of nonlinear dynamical systems: chaos, fractals, self-similarity and the limits of prediction Systems 2016;4:37. https://doi.org/10.3390/systems4040037

124. Alonso C, Pérez R, Bazaga P, Herrera CM. Global DNA cytosine methylation as an evolving trait: phylogenetic signal and correlated evolution with genome size in angiosperms. Frontiers in Genetics 2015;6(4):19. https://doi.org/10.3389/fgene.2015.00004

125. Autzen B. Bayesian convergence and the fair-balance paradox. Erkenntnis 2018;83:23-263. https://doi.org/10.1007/s10670-017-9888-0

126. Yang Z, Zhu T. Bayesian selection of misspecified models is overconfident and may cause spurious posterior probabilities for phylogenetic trees. Proc Nat Acad Sci USA 2018;115:1854-1859. https://doi.org/10.1073/pnas.1712673115

127. Sullivan J, Swofford DL. Should we use model-based methods for phylogenetic inference when we know that assumptions about among-site rate variation and nucleotide substitution pattern are violated? Syst Biol. 2001;50:723-729. http://www.jstor.org/stable/3070810

128. Hershkovitz MA, Zimmer EA. Ribosomal DNA evidence and disjunctions of western American Portulacaceae. Mol Phyl Evol. 2000;15:419-439. https://doi.org/10.1006/mpev.1999.0720

129. Gillespie RG, Baldwin BG, Waters JM, Fraser CI, Nikula R, Roderick GK. Long-distance dispersal: a framework for hypothesis testing. TREE 2012;27:47-56. https://doi.org/10.1016/j.tree.2011.08.009 
Hershkovitz

Montiaceae Ecological Evolution

130. Nathan R, Schurr FM, Spiegel O, Steinitz O, Trakhtenbrot A, Tsoar A. Mechanisms of long-distance seed dispersal. TREE 2008;23:638-647. https://doi.org/10.1016/j.tree.2008.08.003

131. Travisano M, Mongold JA, Bennet AF, Lenski RE. Experimental tests of the roles of adaptation, chance, and history in evolution. Science 1995;267:87-90. https://doi.org/10.1126/science.7809610

132. Travisano M, Vasi F, Lenski RE. Long-term experimental evolution in Escherichia coli III: Variation among replicate populations in correlated responses to novel environments. Evolution 1995;49:189-200. https://www.jstor.org/stable/2410304

133. Woods RJ, Barrick JE, Cooper TF, Shrestha U, Kauth MR, Lenski RE (2011) Second-order selection for evolvability in a large Escherichia coli population. Science 2011;331:1433-1436. https://doi.org/10.1126/science.1198914

134. Wagner GP, Altenberg L. Complex adaptations and the evolution of evolvability. Evolution 1996;50:967976. https://doi.org/10.1111/j.1558-5646.1996.tb02339.x

135. Wagner A. Robustness and evolvability: a paradox resolved. Proc Biol Sci. 2008;275:91-100. https://doi.org/10.1098/rspb.2007.1137

136. Edwards EJ, Chatelet DS, Spriggs EL, Johnson ES, Schultius C, Donoghue MJ. Correlation, causation, and the evolution of leaf teeth: a reply to Givnish and Kriebel. Amer J Bot. 2017;104:509-515. https://doi.org/10.3732/ajb.1700075

137. Packard A. No need for dualism in evolutionary theory: a comment on Cobb JB 'A fourth variable in evolution'. In: Koutroufinis SA, editor. Life and process: towards a new biophilosophy. Berlin: De Gruyter; 2014. p. 225-246.

138. Wilson E0. Consilience: the unity of knowledge. New York: Knopf; 1998.

139. Alicea B, Gordon R. Toy models for macroevolutionary patterns and trends. BioSystems 2014;122:25-37. http://dx.doi.org/10.1016/j.biosystems.2014.06.012

140. Gunawardena J. Models in biology: 'accurate descriptions of our pathetic thinking.' BMC Biology 2014;12:29. http://www.biomedcentral.com/1741-7007/12/29

141. Gerhold P, Cahill Jr JF, Winter M, Bartish IV, Prinzing A. Phylogenetic patterns are not proxies of community assembly mechanisms (they are far better). Functional Ecol. 2015;29:600-614. https://doi.org/10.1111/1365-2435.12425

142. Ricklefs RE. Disintegration of the ecological community: American Society of Naturalists Sewall Wright Award winner address. Amer Nat. 2008;172:741-750. https://doi.org/10.1086/593002 